\title{
A Reduced Order Approach for Probabilistic Inversions of 3D Magnetotelluric Data I: General Formulation
}

\author{
M. C. Manassero ${ }^{1}$, J. C. Afonso ${ }^{1,2}$, F. Zyserman ${ }^{3}$, S. Zlotnik ${ }^{4}$ and I. Fomin ${ }^{1}$ \\ ${ }^{1}$ Australian Research Council Centre of Excellence for Core to Crust Fluid Systems/GEMOC, Department of \\ Earth and Environmental Sciences, Macquarie University, Sydney, Australia. \\ ${ }^{2}$ Centre for Earth Evolution and Dynamics, Department of Geosciences, University of Oslo, Norway. \\ ${ }^{3}$ CONICET, Facultad de Ciencias Astronómicas y Geofísicas, Universidad de La Plata, Argentina. \\ ${ }^{4}$ Laboratori de Càlcul Numèric, Escola Tècnica Superior d'Enginyers de Camins, Canals i Ports, Universitat \\ Politècnica de Catalunya, Barcelona, Spain.
}

\begin{abstract}
SUMMARY
Simulation-based probabilistic inversions of 3D magnetotelluric (MT) data are arguably the best option to deal with the non-linearity and non-uniqueness of the MT problem. However, the computational cost associated with the modeling of 3D MT data has so far precluded the community from adopting and/or pursuing full probabilistic inversions of large MT datasets. In this contribution, we present a novel and general inversion framework, driven by Markov chain Monte Carlo (MCMC) algorithms, which combines i) an efficient parallel-in-parallel structure to solve the 3D forward problem, ii) a reduced order technique to create fast and accurate surrogate models of the forward problem, and iii) adaptive strategies for both the MCMC algorithm and the surrogate model. In particular, and contrary to traditional implementations, the adaptation of the surrogate is integrated into the MCMC inversion. This circumvents the need of costly offline stages to build the surrogate and further increases the overall efficiency of the method.
\end{abstract}




\section{M.C. Manassero et al.}

We demonstrate the feasibility and performance of our approach to invert for large-scale conductivity structures with two numerical examples using different parameterizations and dimensionalities. In both cases, we report staggering gains in computational efficiency compared to traditional MCMC implementations. Our method finally removes the main bottleneck of probabilistic inversions of 3D MT data and opens up new opportunities for both stand-alone MT inversions and multi-observable joint inversions for the physical state of the Earth's interior.

Key words: Magnetotellurics - Composition and structure of the mantle - Inverse TheoryReduced Order modeling - Numerical Modeling - Numerical approximations and Analysis.

\section{INTRODUCTION}

The magnetotelluric (MT) method (Cagniard, 1953; Tikhonov, 1950; Vozoff, 1990) is a passive electromagnetic technique that aims to determine the electrical conductivity distribution of the subsurface at depths from tens of meters to hundreds of kilometers (Vozoff, 1990). Given the strong sensitivity of the electrical conductivity of rocks to temperature, hydrogen and fluid/melt content inside the Earth (Evans, 2012; Karato, 1990; Wang et al., 2006; Yoshino et al., 2012; Yoshino, 2010; Khan, 2016; Roberts \& Tyburczy, 1999; Ten Grotenhuis et al., 2005; Dai \& Karato, 2009; Yoshino et al., 2009; Karato, 2011; Karato \& Wang, 2013; Selway, 2014), MT has become one of the most useful and costefficient techniques to identify deep fluid pathways and associated mineralization events (e.g. Wei et al., 2001; Ogawa et al., 2001), characterize pluming systems in active volcanic areas (e.g. Heise et al., 2008; Comeau et al., 2016), detect partial melting in the deep mantle (e.g. Evans et al., 2005; Selway et al., 2019), and study the thermochemical structure of the lithosphere in general (e.g. Meqbel et al., 2014; Jones et al., 2017; Fullea et al., 2011). These and other features of MT gave impetus for recent data acquisition programs over large grids such as the magnetotelluric component of the USArray in US, the AusLAMP program in Australia and the MT component of Sinoprobe in China. Similarly, the availability of high-quality magnetic field measurements with uniform global coverage done by satellite missions (e.g., see Sabaka et al., 2004; Olsen et al., 2006, 2013; Kuvshinov, 2008) makes it now possible to study lithospheric and whole-mantle conductivity anomalies at global scale (e.g. Kelbert et al., 2009; Kuvshinov \& Olsen, 2006; Kuvshinov et al., 2006; Kuvshinov \& Semenov, 2012; Semenov \& Kuvshinov, 2012).

The formal combination of MT measurements with gravity and seismic data via joint inversions 
(e.g. Moorkamp et al., 2007, 2010; Jones et al., 2017; Vozar et al., 2014; Bennington et al., 2015; Jegen et al., 2009) also holds great potential, as these data sets have complementary sensitivities to the physical state of the lithosphere. For instance, specific types of seismic data can be used in combination with gravity observations to provide the background (or regional) temperature structure and compositional layering (Afonso et al., 2013a,b, 2016a; Jegen et al., 2009; Khan et al., 2008). By accounting for the effect of this background in MT data, conductivity anomalies associated with factors other than temperature and bulk composition (e.g. fluid content, presence of melt, anomalous mineral assemblages) could be identified more reliably.

Due to the potential of MT methods, significant efforts have been made in the past two decades to develop robust and efficient forward and inversion algorithms (e.g. Newman \& Alumbaugh, 2000; Zhdanov et al., 2000; Sasaki, 2001; Siripunvaraporn et al., 2005; Siripunvaraporn \& Egbert, 2009; Han et al., 2008; Egbert \& Kelbert, 2012; Newman \& Alumbaugh, 2000; Mackie \& Madden, 1993; Farquharson et al., 2002; deGroot Hedlin \& Constable, 1990; Key, 2016; Farquharson et al., 2002; Avdeev \& Avdeeva, 2009). In particular, deterministic methods have been successfully applied in the inversion of high-dimensional MT problems and currently represent the most widely used techniques for inverting 3D MT data (Robertson et al., 2020; Meqbel et al., 2014; Yang et al., 2015; Zhdanov et al., 2010; Tietze \& Ritter, 2013; Mackie et al., 1996). Some popular 3D deterministic inversion algorithms include those of Mackie \& Madden (1993), Newman \& Alumbaugh (2000); Newman \& Boggs (2004), Kelbert et al. (2008, 2014); Siripunvaraporn et al. (2005); Siripunvaraporn \& Egbert (2009); Egbert \& Kelbert (2012), based on finite difference forward solvers; the algorithms developed by Zhdanov \& Hursan (2000), Zhdanov et al. (2000) and Avdeev \& Avdeeva (2009), based on an integral equation formulation of the forward solver; and that of Haber et al. (2004), based on a finite element formulation. A review of the different methods available for inversion of 3D MT data can be found in Avdeev (2005) and Siripunvaraporn (2012).

The main advantage of deterministic inversions is that they typically require only a few tens or hundreds of forward evaluations to achieve convergence. This is particularly important to make largescale 3D MT inversions practical, as the forward problem is notoriously computationally expensive. However, deterministic solutions of non-linear problems often depend on the choice of the initial model (to be perturbed during the inversion) and on arbitrary regularization parameters to smoothout the solution (e.g. Robertson et al., 2020). It is well known that if our bias is inappropriate for the problem at hand, the regularized solution can be far from the true solution (Shen et al., 2012; Aster et al., 2018). In addition, the linearization of the problem and optimization algorithms used in determinisitc inversions are tailored for the search of a single optimal or best-fitting model. This 


\section{4}

M.C. Manassero et al.

makes it difficult, if not impossible, to perform thorough statistical analysis of model uncertainties and (perhaps more importantly) non-uniqueness.

Probabilistic inversion methods (Tarantola, 2005; Mosegaard \& Hansen, 2016; Gregory, 2005) are better suited to handle these limitations. They do not focus on single best solutions but rather on providing a full probability distribution over the whole model parameter space. This distribution, known as the posterior distribution, contains all the information about the unknown parameters given data and modeling assumptions and thus, it represents the most general solution to the inverse problem. The main benefits of probabilistic solutions to the MT inverse problem (including full consideration of its nonlinear nature) have been recently highlighted by Conway et al. (2018), Brodie \& Jiang (2018), Jones et al. (2017), Chen et al. (2012) and Rosas-Carbajal et al. (2013) in the context of 1D and 2D inversions driven by Markov Chain Monte Carlo (MCMC) algorithms. However, for the case of high-dimensional 3D MT inversions, the large number of costly forward computations required ( $10^{5}-10^{7}$ ) has so far rendered it impractical. For instance, Rosas-Carbajal et al. (2015) performed a time-lapse 3D MCMC inversion using a Legendre moment decomposition of a saline plume to reduce the dimensionality of the inverse problem to only fourteen parameters. Despite this drastic reduction of the parameter space, convergence was achieved after 60 days of computation.

It is clear that if fully probabilistic inversions of high-dimensional 3D MT problems are to become a reality, significantly more efficient (yet accurate) forward solvers need to be developed. This is precisely the purpose of this work, which presents a new reduced order strategy to solve the probabilistic 3D MT inverse problem. The new approach is based on a judicious combination of a reduced order technique (Reduced Basis Method) to construct fast and accurate surrogate models, an efficient parallel-in-parallel structure of the 3D forward problem and adaptive strategies for both the surrogate and the MCMC algorithm. We will illustrate the feasibility and general performance of our approach with two numerical examples of complex, lithospheric-scale conductivity structures. In particular, we will demonstrate that probabilistic inversions of high-dimensional 3D MT problems are now a practical option, even with modest computational resources.

The remainder of the paper is structured as follows: Section 2 reviews the general formulation of the probabilistic inverse problem. Section 3 introduces the forward problem in terms of primary and secondary fields, and the associated variational formulation. Sections 4 and 5 describe the Reduced Basis Method and our combined Reduced Basis-Markov Chain Monte Carlo strategy (RB+MCMC), respectively. Section 6 presents numerical examples that illustrate the benefits and limitations of the method in the context of stand-alone 3D MT inversions for large-scale lithospheric structures. Section 7 deals with practical aspects of our approach, further improvements and remaining challenges. Lastly, the main outcomes and results of this work are summarized in Section 8. 


\section{PROBABILISTIC INVERSE PROBLEMS}

\subsection{Bayesian Inversion}

The Bayesian approach (sometimes referred to as the statistical or probabilistic approach) to the inverse problem recognizes that the data $(\mathbf{d})$ and the model parameters $(\mathbf{m})$ that characterize the system will never be known with absolute certainty and it considers them to be random variables represented by associated probability distributions (e.g. Tarantola et al., 1982; Mosegaard \& Hansen, 2016; Gregory, 2005; Gilks et al., 1995; Sen \& Stoffa, 1996). The most general solution to the inverse problem is therefore a posterior probability density function (PDF) over the parameter space which is formally given by the Bayes' rule

$$
P(\mathbf{m} \mid \mathbf{d})=\frac{P(\mathbf{d} \mid \mathbf{m}) P(\mathbf{m})}{P(\mathbf{d})},
$$

where $P(\mathbf{m} \mid \mathbf{d})$ is the posterior conditional PDF of $\mathbf{m}$ given $\mathbf{d} . P(\mathbf{d} \mid \mathbf{m})$ is the conditional PDF of $\mathbf{d}$ given $\mathbf{m}, P(\mathbf{m})$ is the prior PDF of the model parameters $\mathbf{m}$ and $P(\mathbf{d})$ is the prior PDF of the data. When a particular observation is made, $P(\mathbf{d} \mid \mathbf{m})$ is considered a function of $\mathbf{m}$ and referred to as the likelihood, $\mathcal{L}(\mathbf{m})$. Considering this and the fact that $P(\mathbf{d})$ is independent of $\mathbf{m}$, Eq.1 can be written as

$$
P(\mathbf{m} \mid \mathbf{d}) \propto \mathcal{L}(\mathbf{m}) P(\mathbf{m}) .
$$

In the most general case of high-dimensional and non-linear problems with complex priors, the only practical solution for Eq. 2 is based on the construction of a Markov chain that has $P(\mathbf{m} \mid \mathbf{d})$ as their equilibrium or stationary distribution (Tarantola, 2005; Gregory, 2005; Gilks et al., 1995). Markov chain Monte Carlo (MCMC) algorithms are designed to produce unbiased approximations of the true posterior by repeatedly drawing models $\mathbf{m}_{t}$ and evaluating their posterior probability $P\left(\mathbf{m}_{t} \mid \mathbf{d}\right)$.

In this work, we use the Metropolis-Hastings (MH) algorithm (Metropolis et al., 1953; Hastings, 1970) combined with the adaptive Metropolis (AM) approach of Haario et al. (2001). At the core of these algorithms is a proposal distribution, $q(\cdot \mid \cdot)$, used to generate new moves or trials. In principle, the proposal distribution can be chosen rather freely from a large family of distributions (cf. Gregory, 2005; Gilks et al., 1995). In practice, however, the actual form of $q(\cdot \cdot \cdot)$ exerts a strong influence on the efficiency of the MCMC algorithm. The AM algorithm alleviates the problem of choosing an optimal proposal before the MCMC simulation starts by updating the proposal at regular intervals according to the statistics of the chain. The reader is referred to Haario et al. (2001) for further details on the AM algorithm.

At each step of the MCMC simulation, a new move in the parameter space $\left(\mathbf{m}_{t}\right)$ is proposed from $q(\cdot \mid \cdot)$ and its posterior probability is compared to that of the current state of the chain $\left(\mathbf{m}_{t-1}\right)$. If the 
new proposal has a higher probability than the current state, it is accepted as part of the chain and the current position is updated to the new state. If the new proposal has a lower probability than the current state, it is accepted with probability $\alpha\left(\mathbf{m}_{t-1}, \mathbf{m}_{t}\right)$, defined as

$$
\alpha\left(\mathbf{m}_{t-1}, \mathbf{m}_{t}\right)=\min \left\{1, \frac{\mathcal{L}\left(\mathbf{m}_{t}\right) P\left(\mathbf{m}_{t}\right) q\left(\mathbf{m}_{t-1} \mid \mathbf{m}_{t}\right)}{\mathcal{L}\left(\mathbf{m}_{t-1}\right) P\left(\mathbf{m}_{t-1}\right) q\left(\mathbf{m}_{t} \mid \mathbf{m}_{t-1}\right)}\right\} .
$$

where we have assumed that the new proposal is conditional on the current state of the chain (Gregory, 2005; Gilks et al., 1995). Details about the proposal distribution and the AM algorithm relevant to this work can be found in Appendix B.

\subsection{The Likelihood Function}

The likelihood function is a measure of the distance between the observed data and the solution of the forward model, i.e it is determined by the statistical distribution of the data errors. Under the common assumption of additive independent Gaussian errors, the likelihood function is given by:

$$
\mathcal{L}(\mathbf{m} \mid \mathbf{d})=\frac{1}{(2 \pi)^{N / 2} \prod_{i=1}^{N} s_{i}} \exp \left[-\frac{1}{2} \sum_{i=1}^{N}\left(\frac{g_{i}(\mathbf{m})-d_{i}}{s_{i}}\right)^{2}\right],
$$

where $N$ is the number of independent observations, $s_{i}$ denotes the standard deviation of the $i$-th data error and $\mathbf{g}(\mathbf{m})$ is the data predicted by the forward problem for the model $\mathbf{m}$.

\section{THE MAGNETOTELLURIC FORWARD PROBLEM}

\subsection{Magnetotelluric Equations}

The basis of the magnetotelluric (MT) method is the electromagnetic (EM) induction problem, viz. EM fields are induced within the Earth by EM fields that propagate perpendicular to the surface of the Earth as a plane monochromatic wave of frequency $\omega$. The induced EM fields satisfy the following time-harmonic Maxwell's partial differential equations:

$$
\begin{aligned}
\nabla \times \mathbf{H} & =\sigma \mathbf{E}, \\
\nabla \times \mathbf{E} & =-i \omega \mu_{0} \mathbf{H},
\end{aligned}
$$

where $\mathbf{E}$ is the electric field $[\mathrm{V} / \mathrm{m}], \mathbf{H}$ is the magnetic field $[\mathrm{A} / \mathrm{m}], \mu_{0}$ is the magnetic permeability of free space $[V s / A m]$ and $\sigma$ is the electrical conductivity of the medium $[S / m]$.

\subsection{Variational Formulation}

This paper follows the formulation of Zyserman \& Santos (2000) where the electrical conductivity distribution in a domain $\Omega \in \mathbb{R}^{3}$ is considered as the superposition of a background or primary 
conductivity of a layered Earth $\left(\sigma_{p}\right)$ and an additional conductivity associated with the presence of three-dimensional anomalies $\left(\sigma_{s}\right)$. This formulation allows to rewrite Eqs. 5 in terms of primary fields $\left(\mathbf{H}_{p}\right.$ and $\left.\mathbf{E}_{p}\right)$ induced in the layered Earth and secondary fields $\left(\mathbf{H}_{s}\right.$ and $\left.\mathbf{E}_{s}\right)$ which are generated by the presence of the conductivity anomalies. Using the absorbent boundary conditions defined by Sheen (1997), our MT problem in 3D is formulated as follows:

Find $\mathbf{E}_{s}$ and $\mathbf{H}_{s}$ in a domain $\Omega \in \mathbb{R}^{3}$ such that,

$$
\begin{aligned}
\sigma \mathbf{E}_{s}-\nabla \times \mathbf{H}_{s} & =-\mathbf{F}=-\sigma_{s} \mathbf{E}_{p} & & \text { in } \Omega, \\
i \omega \mu_{0} \mathbf{H}_{s}+\nabla \times \mathbf{E}_{s} & =0 & & \text { in } \Omega, \\
(1-i) P_{\tau} a \mathbf{E}_{s}+\nu \times \mathbf{H}_{s} & =0 & & \text { on } \partial \Omega \equiv \Gamma,
\end{aligned}
$$

where $\Gamma \equiv \partial \Omega$ is the boundary of the domain $\Omega$ and $a$ is defined as $a=\left(\sigma / 2 \omega \mu_{0}\right)^{1 / 2}$. In the first term of Eq. $6 \mathrm{c}, P_{\tau} \varphi=\varphi-\nu(\nu \cdot \varphi)$ refers to the projection of the trace of any vector $\varphi$ on $\Gamma$ where $\nu$ is the unit outer normal to $\Gamma$.

Omitting the subscripts for the secondary fields, the variational formulation of the mixed problem solved in Eqs. 6 reads as follows (Douglas Jr et al., 2000):

Find $(\mathbf{E}, \mathbf{H})$ in the suitable spaces $\mathcal{V} \times \mathcal{W}$ such that,

$$
\begin{aligned}
(\sigma \mathbf{E}, \boldsymbol{\varphi})-(\mathbf{H}, \nabla \times \boldsymbol{\varphi})+(1-i)\left\langle P_{\tau} a \mathbf{E}, P_{\tau} \boldsymbol{\varphi}\right\rangle_{\Gamma} & =(\mathbf{F}, \boldsymbol{\varphi}) & & \boldsymbol{\varphi} \in \mathcal{V}, \\
i \omega \mu_{0}(\mathbf{H}, \boldsymbol{\psi})+(\nabla \times \mathbf{E}, \boldsymbol{\psi}) & =0 & & \boldsymbol{\psi} \in \mathcal{W} .
\end{aligned}
$$

\subsection{Numerical Forward Solution}

Numerical solutions to the variational problem in Eqs. 7 are obtained using a nonconforming Galerkin finite element (FE) method (Douglas Jr et al., 1999, 2000; Zyserman \& Santos, 2000). This requires finding $\left(\mathbf{E}^{h}, \mathbf{H}^{h}\right) \in \mathcal{V}^{h} \times \mathcal{W}^{h}$ such that,

$$
\begin{array}{rlrl}
\left(\sigma \mathbf{E}^{h}, \boldsymbol{\varphi}\right)-\sum_{j}^{N_{e}}\left(\mathbf{H}^{h}, \nabla \times \boldsymbol{\varphi}\right)_{\Omega_{j}}+(1-i)\left\langle\left\langle P_{\tau} a \mathbf{E}^{h}, P_{\tau} \boldsymbol{\varphi}\right\rangle\right\rangle_{\Gamma} & =\ell(\boldsymbol{\varphi}) & & \forall \boldsymbol{\varphi} \in \mathcal{V}^{h}, \\
i \omega \mu_{0}\left(\mathbf{H}^{h}, \boldsymbol{\psi}\right)+\sum_{j}^{N_{e}}\left(\nabla \times \mathbf{E}^{h}, \boldsymbol{\psi}\right)_{\Omega_{j}}=0 & \forall \boldsymbol{\psi} \in \mathcal{W}^{h} .
\end{array}
$$

where $N_{e}$ is the number of non-overlapping parallelepipeds $\Omega_{j}$ such that $\Omega=\cup_{j} \Omega_{j}, j=1, \ldots, N_{e} ; \varphi$ and $\psi$ are the set of FE shape functions for the electric and magnetic field, respectively, and $N_{F E}$ and $M_{F E}$ are the degrees of freedom for each field. The finite element subspaces $\mathcal{V}_{h} \subset \mathcal{V}$ and $\mathcal{W}_{h} \subset \mathcal{W}$ are defined as

$$
\begin{aligned}
\mathcal{V}_{h} & =\operatorname{span}\left\{\varphi^{1}, \ldots, \varphi^{N_{F E}}\right\}, \\
\mathcal{W}_{h} & =\operatorname{span}\left\{\psi^{1}, \ldots, \psi^{M_{F E}}\right\} .
\end{aligned}
$$




\section{M.C. Manassero et al.}

The probabilistic behaviour of the 3D MT inverse problem is introduced when we assume that the electrical conductivity is a random field of the form $\sigma(\mathbf{x}, \theta)$ where $\mathbf{x} \in \Omega$ is the vector position and $\theta \in \Theta$ refers to the randomness, being $\Theta$ the set of all possible outcomes of $\theta$. For a specific sample of $\sigma(\mathbf{x}, \theta)$, the approximate electric and magnetic field on each $\Omega_{j}$ can be expressed in terms of the shape functions as follows:

$$
\begin{aligned}
\mathbf{E}^{h}(\mathbf{x}, \theta) & =\sum_{\alpha=1}^{N_{F E}} \varepsilon^{\alpha}(\theta) \boldsymbol{\varphi}^{\alpha}(\mathbf{x}), \\
\mathbf{B}^{h}(\mathbf{x}, \theta) & =\sum_{\eta=1}^{M_{F E}} h^{\eta}(\theta) \boldsymbol{\psi}^{\eta}(\mathbf{x}),
\end{aligned}
$$

where $\varepsilon^{\eta}(\theta)$ and $h^{\alpha}(\theta)$ are the unknown coefficients to be determined for a that sample $\sigma(\mathbf{x}, \theta)$.

Introducing Eqs. 10 and 11 into Eqs. 8 and selecting one shape function at the time $\varphi^{n}, n=$ $1, . ., N_{F E}$ and $\psi^{m}, m=1, . ., M_{F E}$ we obtain the following algebraic system of equations for the sample $\sigma(\mathbf{x}, \theta)$ :

$$
\begin{gathered}
\left(\sigma(\mathbf{x}, \theta) \sum_{\alpha=1}^{N_{F E}} \varepsilon^{\alpha}(\theta) \boldsymbol{\varphi}^{\alpha}(\mathbf{x}), \boldsymbol{\varphi}^{n}(\mathbf{x})\right)-\sum_{j}^{N_{e}}\left(\sum_{\eta=1}^{M_{F E}} h^{\eta}(\theta) \boldsymbol{\psi}^{\eta}(\mathbf{x}), \nabla \times \boldsymbol{\varphi}^{n}(\mathbf{x})\right)_{\Omega_{j}} \\
+(1-i)\left\langle\left\langle P_{\tau} a \sum_{\alpha=1}^{N_{F E}} \varepsilon^{\alpha}(\theta) \boldsymbol{\varphi}^{\alpha}(\mathbf{x}), P_{\tau} \boldsymbol{\varphi}^{n}(\mathbf{x})\right\rangle\right\rangle_{\Gamma}=\ell\left(\boldsymbol{\varphi}^{n}(\mathbf{x})\right) \forall \boldsymbol{\varphi}^{n}, \\
i \omega \mu_{0}\left(\sum_{\eta=1}^{M_{F E}} h^{\eta}(\theta) \boldsymbol{\psi}^{\eta}(\mathbf{x}), \boldsymbol{\psi}^{m}(\mathbf{x})\right)+\sum_{j}^{N_{e}}\left(\nabla \times \sum_{\alpha=1}^{N_{F E}} \varepsilon^{\alpha}(\theta) \boldsymbol{\varphi}^{\alpha}(\mathbf{x}), \boldsymbol{\psi}^{m}(\mathbf{x})\right)_{\Omega_{j}}=0 \forall \boldsymbol{\psi}^{\boldsymbol{m}} .
\end{gathered}
$$

Eqs. 12 can be further simplified as the choice of the shape functions establishes a relationship between $h^{\eta}$ and $\varepsilon^{\alpha}$ (see Douglas Jr et al., 2000; Zyserman \& Santos, 2000). When computing the integral over an element $\Omega_{j}$, the first term of Eq. $12 \mathrm{~b}$ is different from zero only when $\eta=m$ allowing the coefficients $h^{\eta}$ of Eq. $12 \mathrm{~b}$ to be be expressed in terms of $\varepsilon^{\alpha}$ and replaced in Eq. 12a. This simplification results in a linear system of equations where the unknown is a vector $\mathbf{U}$ with the coefficients $\varepsilon^{\alpha}$ for the approximated electric field in the whole domain. This system of equations can be represented in the following matrix form:

$$
\mathbb{K}(\theta) \mathbf{U}(\theta)=\mathbf{F}(\theta),
$$

where $\mathbb{K}$ is a sparse and symmetric stiffness matrix of size $N_{F E} \times N_{F E}$ and $\mathbf{F}$ is the force vector of size $N_{F E} \times 1$.

In our implementation of the original code of Zyserman \& Santos (2000), the system of Eqs. 13 is solved using the MUlti-frontal Massively Parallel Solver (MUMPS) version 5.1.2 (Amestoy et al., 2001, 2006). This allows us to define an efficient parallel-in-parallel structure. The first level of 
parallelization involves assigning a block of $n p$ processors to each frequency (i.e. parallelization by frequencies). The second level involves parallelizing the solution of the actual forward problem (Eqs. 13) for each frequency using MUMPS and the $n p$ processors in each block. This structure reduces the computational time of each forward solution by $\sim 80 \%$, regardless of the number of frequencies used. Results for standard 3D MT benchmarks using this implementation are provided in Section 1 of the Supplementary Material.

Despite the parallel strategy described above, the computational time taken by the forward problem remains the main limitation preventing its use in MCMC-driven probabilistic inversions of 3D MT data. The following section presents a novel strategy based on reduced order modeling that overcomes this limitation.

\section{REDUCED BASIS}

In order to ameliorate the computational burden of probabilistic inversions and optimization problems, various methods for reducing the cost of the full (high-fidelity) forward problem have been proposed (see reviews in Peherstorfer et al., 2018; Frangos et al., 2010). Among all available techniques, the projection-based Reduced Basis (RB) method (Florentin \& Díez, 2012; Hesthaven et al., 2016; Quarteroni et al., 2015; Patera et al., 2007) is particularly well suited for our purposes due to its strong connection to FE discretizations, its implementation simplicity and its outstanding performance in situations where the same physical problem needs to be solved many times for different input parameters (as is the case in MCMC-driven inversions). In the RB method, the solution of every new model (i.e. forward problem) is sought as a projection onto a space built from previous high-fidelity solutions called bases. The space containing these high-fidelity solutions is referred to as the reduced basis. Previous work has shown that RB can speedup the numerical solutions of complex problems by several orders of magnitude without compromising the accuracy of the solutions (e.g. Ortega-Gelabert et al., 2020; Lieberman et al., 2010; Chen et al., 2010; Rozza et al., 2007, 2013; Florentin \& Díez, 2012; Cui et al., 2015). In this paper, we will show that staggering gains in computational time can also be achieved for the 3D MT problem. The reader is referred to Quarteroni et al. (2015, 2011), Benner et al. (2017) and Hesthaven et al. (2016) for more details on RB methods and their applications to a range of problems.

\subsection{Reduced Basis Solution}

The Reduced Basis (RB) approach used in this paper (cf. Florentin \& Díez, 2012) disassociates the discretization space $(\Omega)$ and the stochastic space $(\Theta)$ in a sense that the spatial discretization used 
to find the high-fidelity FE solution is not allowed to vary across the MCMC simulations, i.e. the stochasticity is only assigned to the conductivity distribution, not to the FE discretization. Since the coefficients $h^{\eta}$ for the magnetic field can be expressed as functions of the coefficients $\varepsilon^{\alpha}$ for the electric field, the low-fidelity RB solution, $\mathbf{U}_{\mathbf{R B}}$, seeks to approximate the electric field only,

$$
\mathbf{U}_{\mathbf{R B}}(\theta) \approx \mathbf{U}
$$

The main idea behind the RB method is to generate a subset $\mathcal{V}_{\mathcal{R B}}$ of $\mathcal{V}_{h}$ with dimension $N_{R B} \ll$ $N_{F E}$ such that,

$$
\mathcal{V}_{\mathcal{R B}}=\operatorname{span}\left\{\mathbf{V}_{1}, \mathbf{V}_{2}, \ldots, \mathbf{V}_{N_{R B}}\right\} \subset \mathcal{V}_{h}
$$

where the associated basis vectors $\mathbf{V}_{i}$ are defined as high-fidelity FE solutions of the system of Eqs. 13 for certain realizations of $\sigma(\mathbf{x}, \theta)$. The generation of these bases will be described in Section 5 and we assume, for the time being, that we have an available $\mathcal{V}_{\mathcal{R B}}$. While performing the probabilistic inversion, the solution of Eq. 13 for a new sample $\sigma(\mathbf{x}, \theta)$ is first sought as a linear combination of the basis vectors in $\mathcal{V}_{\mathcal{R B}}$ :

$$
\mathbf{U}_{\mathbf{R B}}(\mathbf{x}, \theta)=\sum_{i=1}^{N_{R B}} a_{i}(\theta) \mathbf{V}_{i}=\mathbb{V}_{\mathbb{R B}} \mathbf{a}(\theta)
$$

where $\mathbb{V}_{\mathbb{R B}}=\left[\mathbf{V}_{1}, \mathbf{V}_{2}, \ldots, \mathbf{V}_{N_{R B}}\right]^{N_{F E} \times N_{R B}}$ and $\mathbf{a}(\theta)=\left[a_{1}, a_{2}, \ldots a_{N_{R B}}\right]$ is the vector of unknown coefficients accompanying the bases $\mathbf{V}_{i}$.

Introducing $\mathbf{U}_{\mathbf{R B}}(\mathbf{x}, \theta)$ into Eq. 13 and multiplying both sides of the equation by $\mathbb{V}_{R B}^{T}$ we obtain:

$$
\left(\mathbb{V}_{\mathbb{R B}}^{T} \mathbb{K}(\theta) \mathbb{V}_{\mathbb{R B}}\right) \mathbf{a}(\theta)=\mathbb{V}_{\mathbb{R B}}^{T} \mathbf{F}(\theta)
$$

Defining $\mathbb{K}_{\mathbb{R B}}(\theta):=\mathbb{V}_{\mathbb{R B}}^{T} \mathbb{K}(\theta) \mathbb{V}_{\mathbb{R} \mathbb{B}}$ and $\mathbf{F}_{\mathbf{R B}}(\theta):=\mathbb{V}_{\mathbb{R B}}{ }^{T} \mathbf{F}(\theta)$, we can rewrite Eq. 17 to obtain the following system of equations:

$$
\mathbb{K}_{\mathbb{R B B}}(\theta) \mathbf{a}(\theta)=\mathbf{F}_{\mathbf{R B}}(\theta),
$$

where $\mathbb{K}_{\mathbb{R B B}}$ is the reduced basis matrix of size $N_{R B} \times N_{R B}$ and $\mathbf{F}_{\mathbf{R B}}$ is the force vector of size $N_{R B} \times 1$.

The RB solution, $\mathbf{U}_{\mathbf{R B}}(\theta)$, for a particular sample $\sigma(\mathbf{x}, \theta)$ is then found by solving the system of Eqs. 18 for the coefficients $\mathbf{a}(\theta)$ and substituting them into Eq. 16. Note that the linear system of Eqs. 18 is of size $N_{R B} \ll N_{F E}$ and thus significantly less expensive than the complete FE system of Eqs. 13 of size $N_{F E}$. 


\subsection{Error Estimation}

Once the RB solution is obtained for a particular sample $\sigma(\mathbf{x}, \theta)$ and a particular basis $\mathbb{V}_{\mathbb{R} \mathbb{B}}$, it is necessary to evaluate whether the basis was sufficiently rich to produce an accurate RB solution. Much work has been done to define reliable error estimators and to certify the accuracy of the RB solution (Hesthaven et al., 2012, 2016; Chen et al., 2010; Quarteroni et al., 2011). In this paper, we define the approximation error between the RB and the FE solution at every step of the MCMC simulation as:

$$
\mathbf{E}_{\mathbf{R B}}:=\mathbf{U}_{\mathbf{R B}}-\mathbf{U}
$$

Since $\mathbf{U}$ is the high-fidelity FE solution that we do not want to compute, we multiply $\mathbf{E}_{\mathbf{R B}}$ by the stiffness matrix $\mathbb{K}$ to obtain the so-called residual of the RB solution:

$$
\mathbb{K} \mathbf{E}_{\mathbf{R B}}=\mathbb{K} \mathbf{U}_{\mathbf{R B}}-\mathbb{K} \mathbf{U}
$$

Replacing Eq. 13 into Eq. 20, dividing by the vector of forces $\mathbf{F}$ and computing the $L_{2}$ norm, we obtain the following RB relative error:

$$
\mathbf{R}_{\mathbf{R B}}:=\frac{\left\|\mathbb{K} \mathbf{U}_{\mathbf{R B}}-\mathbf{F}\right\|}{\|\mathbf{F}\|}
$$

which is an adequate estimator of the energy norm of the RB solution error (Hesthaven et al., 2016; Quarteroni et al., 2015). At this stage, a criterion is necessary to evaluate the accuracy of the approximation. The simplest criterion involves defining a tolerance $\beta$ in a way that the RB solution becomes admissible only if $\mathbf{R}_{\mathbf{R B}} \leq \beta$. In the case of a relative error larger than $\beta$, the RB solution is considered unacceptable and the high-fidelity FE solution is computed. This new FE solution is added to the space $\mathcal{V}_{\mathcal{R B}}$ (i.e. enrichment of $\mathcal{V}_{\mathcal{R} \mathcal{B}}$ ), which is then used in subsequent MCMC steps. This process is further discussed in the following section.

\section{A COMBINED RB+MCMC METHOD FOR 3D MT INVERSION PROBLEMS}

In traditional implementations of RB methods, the creation of the basis (computationally expensive as it involves many solutions of the high-fidelity FE problem) is computed in an offline stage at specific locations within the parameter space defined by an heuristic criterion or greedy procedure (Rozza et al., 2009, 2007). During the subsequent probabilistic inversion or simulation process, known as the online stage, these bases are used to obtain the fast low-fidelity RB solutions (Hesthaven et al., 2016; Prud'Homme et al., 2002; Quarteroni et al., 2015; Hess \& Benner, 2013; Hess, 2016). This type of approach is particularly useful when the number of parameters is small and when only a small number of high-fidelity solutions are able to represent the complete parameter space.

In the context of high- and ultra-high-dimensional ( $>10^{3}$ parameters) probabilistic inversions, it 
is practically impossible to pre-explore the parameter space in an offline stage to create relevant bases that will guarantee accurate solutions within the entire parameter space, but most importantly, within the (so far unknown) high-probability regions. Put differently, in order to guide the creation of the relevant basis, we would have to know a priori the regions of high probability, which implies that we already have a solution to the inverse problem. While this is in principle possible by splitting the inversion into three stages - e.g. an initial low-dimensional (fast) deterministic inversion, the offline creation of the relevant basis and a final MCMC inversion with the RB surrogate -, in the most general case, an adaptive MCMC approach where the basis is computed and adapted during the MCMC simulation seems to be a more effective concept. This is particularly relevant to our goals because of the intrinsic behaviour of MCMC inversions, which typically start in a region of low probability (i.e. bad models) and quickly converge to a restricted region in the parameter space where the most probable models reside. Therefore, the RB solutions that may have been relevant to the initial stages of the chain will become less relevant when the chains have converged to the high probability regions. For the same reason, fewer basis enrichment (high-fidelity solutions) are needed as the chain converges, because the $\mathrm{RB}$ space becomes rich enough to provide good solutions within the restricted parameter space. Florentin \& Díez (2012), Cui et al. (2015), Yan \& Zhou (2019),Zhang et al. (2019) and Ortega-Gelabert et al. (2020) have introduced similar ideas. Ortega-Gelabert et al. (2020), in particular, demonstrated the benefits of this type of RB adaptation and applied it in MCMC inversions of complex geodynamic problems. We emphasize that this approach does not preclude the option of using bases created in previous simulations. Indeed, bases generated during a previous inversion or during a parallel MCMC chain (if different chains can exchange information) can be easily re-used to improve the performance of both subsequent and simultaneous parallel inversions.

\subsection{A Hybrid Approach for High-dimensional Probabilistic Inversions}

Our hybrid approach is similar to that put forward by Ortega-Gelabert et al. (2020) in that it automatically updates/enriches the RB space $\mathcal{V}_{R B}$ during the MCMC inversion by adding high-fidelity solutions (i.e. new bases) as needed by the evolution of the chain. The main differences between the two approaches are in the way they estimate the error introduced by the RB approximation and in the implementation of the adaptive scheme for both the basis and the MCMC algorithms. For instance, our parallel structure allows us to create and adapt the basis per frequency and orientation of the EM fields. This adaptation is also controlled by the use of variable tolerance and an orthonormalization procedure (described below). In the case of the MCMC algorithm, we have implemented an Adaptive Metropolis algorithm (Section 6.3) to update the proposal and incorporated a modified ratio that ac- 
counts for Delayed Rejection (Haario et al., 2006; Mira et al., 2001) when a new high-fidelity solution is computed and the proposed sample is rejected (described below).

The basic steps of our RB+MCMC algorithm (Algorithm 1) can be summarized as follows:

(i) In the case of having previous solutions from an offline stage or from previous probabilistic inversions, we proceed to load these solutions as the initial basis $\mathbb{V}_{\mathbb{R} \mathbb{B}}$. Otherwise, we compute the high-fidelity solution of the starting model (i.e. starting point of the Markov chain). In MT, each forward solution requires the computation of two (typically orthogonal) components of the EM fields per frequency $i, i=1, . ., n_{f r e q}$. Here, these orthogonal solutions per frequency are referred to as $S^{i}$ and $S_{\perp}^{i}$.

(ii) At every proposed sample, $\mathbf{m}_{t}=\sigma_{t}(\mathbf{x}, \theta)$, we compute the fast $\mathrm{RB}$ solutions $\left(\mathbf{U}_{\mathbf{R B}}{ }^{S^{i}}\right.$ and $\left.\mathbf{U}_{\mathbf{R B}}{ }^{S_{\perp}^{i}}\right)$ and their relative $\mathrm{RB}$ errors $\left(\mathbf{R}_{\mathbf{R B}} S^{i}\right.$ and $\left.\mathbf{R}_{\mathbf{R B}}{ }^{S_{\perp}^{i}}\right)$ for all frequencies.

(iii) If all the relative RB errors are smaller than a prescribed tolerance $\beta$, we accept $\mathbf{U}_{\mathbf{R B}}{ }^{S^{i}}$ and $\mathbf{U}_{\mathbf{R B}} S_{\perp}^{i}$ as good approximations of the high-fidelity solution for all frequencies. The corresponding approximate likelihood, $\overline{\mathcal{L}}\left(\mathbf{m}_{t}\right)$, and acceptance probability, $\bar{\alpha}\left(\mathbf{m}_{t-1}, \mathbf{m}_{t}\right)$, are computed and the sample is either accepted or rejected according to the Metropolis-Hastings criterion.

(iv) If any $\mathbf{R}_{\mathbf{R B}}$ is larger than $\beta$, the high-fidelity FE solution for that frequency and component of the EM field is computed at the proposed sample $\mathbf{m}_{t}=\sigma_{t}(\mathbf{x}, \theta)$. This solution is added as a new basis into the corresponding space $\mathcal{V}_{R B}^{S^{i}}$ or $\mathcal{V}_{R B}^{S_{\perp}^{i}}$, thus enriching the RB surrogate.

(v) Since the posterior probabilities of the proposed sample $\mathbf{m}_{\mathbf{t}}$ and that of the current sample $\mathbf{m}_{t-1}$ are no longer comparable (i.e. they were computed with different solvers, FE and RB, respectively), we re-compute $\mathbf{U}_{\mathbf{R B}}{ }^{i_{t-1}}$ (and the associated likelihood) using the newly enriched RB space. If $\mathbf{m}_{t}$ is rejected by the Metropolis-Hastings criterion, a new trial $\mathbf{m}_{t}^{*}$ is proposed in the vicinity of $\mathbf{m}_{t}$ and its likelihood is computed with the newly enriched RB space. This new trial $\mathbf{m}_{t}^{*}$ is accepted/rejected according to a modified Metropolis ratio to account for the delayed rejection (i.e. two proposals) step (see e.g. Haario et al., 2006; Mira et al., 2001).

Step five above is critical to keep the ergodicity of the algorithm. While other choices can be implemented for this purpose, they usually require additional high-fidelity computations at the current state of the chain $\mathbf{m}_{t-1}$ (e.g. Yan \& Zhou, 2019; Ortega-Gelabert et al., 2020). Also, although the delayed rejection step is not strictly necessary from a MCMC algorithmic point of view, it is rather important in practice. This is because, despite the fact that the posteriors of both models $\mathbf{m}_{t}$ and $\mathbf{m}_{t-1}$ have been computed with the same updated surrogate, the surrogate solution for $\mathbf{m}_{t}$ still outputs a "more accurate" high-fidelity (FEM) solution (i.e. the surrogate is effectively a FE solver at $\mathbf{m}_{t}$, but still an approximate surrogate at $\mathbf{m}_{t-1}$ ). We have observed that this tends to decrease the acceptance 


\section{M.C. Manassero et al.}

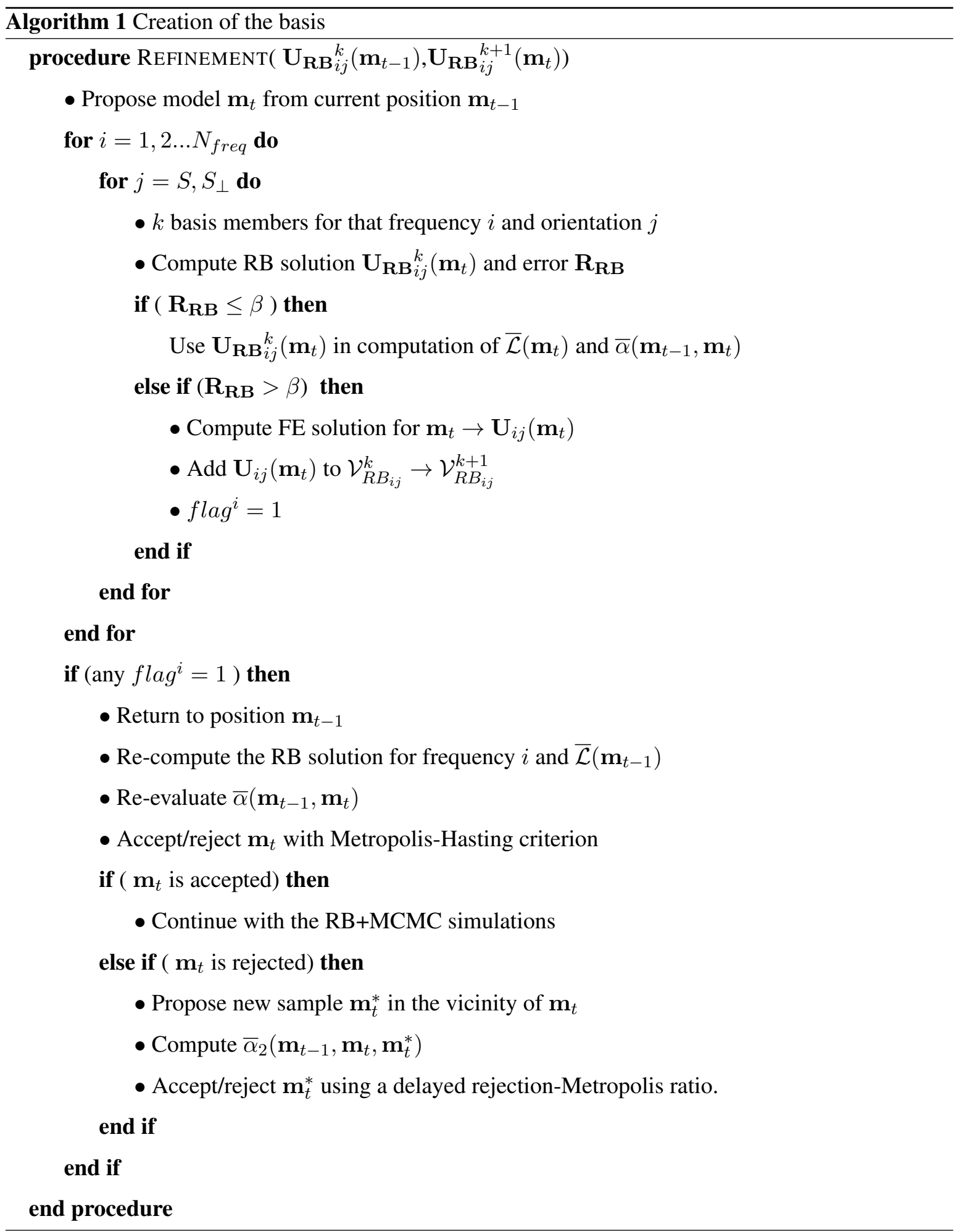

probability of $\mathbf{m}_{t}$ and using a delayed rejection step with a small variance counteracts this undesired effect.

The success of our approach relies on a judicious combination of the Reduced Basis method, the MCMC sampling that drives the generation of bases and the prescribed tolerance $\beta$. These elements 
allow us to adapt and refine the low-fidelity solutions to obtain a very efficient and accurate sampling of the posterior distribution (or equivalently, $\overline{\mathcal{L}}\left(\mathbf{m}_{t}\right)$ tends to the true likelihood $\mathcal{L}\left(\mathbf{m}_{t}\right)$ as the surrogate is adapted). At the same time, it is imperative that the number of elements in the basis is kept as small as possible without compromising the quality of their predictions (cf. Ortega-Gelabert et al., 2020). In order to achieve this, we have implemented the use of variable tolerances and Singular Value Decomposition (SVD) of the basis as additional functionalities.

\subsubsection{Variable tolerance}

During the so-called burn-in stage, a finite number of simulations (and associated forward solutions) is required to move away from the initial low-probability region and towards the support of the posterior PDF. Since these samples are not considered for the statistical analysis of the posterior PDFs, a relatively large tolerance $\beta_{1}>\beta$ can be used during this initial stage to encourage the algorithm to move quickly towards the high probability regions. After this stage, the tolerance can be reduced to guarantee that the sampling within the support of the posterior PDF satisfies the user's criteria for accuracy. Numerous tests (not shown here) and the results in Section 6.3 demonstrate that this simple tactic results in noticeable gains in efficiency during the inversion. While in principle, the actual reduction of the tolerance can be done in any number of steps of decreasing $\beta$, we have not attempted a systematic exploration of all possible combinations. The results presented in Section 6.3 illustrate the use of a one-step reduction (i.e. two tolerance values).

\subsubsection{Decomposition of the basis}

Our reduced basis approach incorporates new solutions into the RB spaces that cannot be explained by the previous basis within a prescribed tolerance. This, however, does not guarantee orthogonality of the individual basis members. A simple way to remove redundancy of information and/or basis members that do not contribute significantly to the RB solution is to apply a SVD procedure (Brunton \& Kutz, 2019). In our algorithm, the SVD is applied to any RB basis space once its size reaches a predefined value $N_{s v d}$. We keep the most relevant $N_{r e l}$ orthogonal elements of the basis up to a pre-defined energy threshold (usually between $0.95 \%$ and $0.999 \%$ ). After the SVD procedure is performed, we re-define a new $N_{s v d}^{*}$ as $N_{s v d}^{*}=N_{s v d}+N_{r e l}$. This redefinition of $N_{s v d}$ allows the size of the basis to progressively increase as needed.

Section 2 of the Supplementary Material presents an application to a simple 1D heat transfer problem. The computational time of this simple forward problem allows us to approximate the true posterior PDFs with high accuracy (using high-fidelity FEM solutions and standard MCMC algorithms) and thus evaluate the accuracy of our RB+MCMC method. 


\section{NUMERICAL EXAMPLES}

In this section, we illustrate the performance of our 3D MT RB+MCMC algorithm by inverting synthetic data corresponding to two complex large-scale lithospheric models with dimensions $1600 \times$ $1600 \times 460 \mathrm{~km}$ (Figs. 1 and 9). In both cases, the computational domain is discretized with $40 \times 40 \times 20$ finite elements.

\subsection{Synthetic Data}

The synthetic data are the off-diagonal apparent resistivities and phases computed for 12 periods between 3.2 and $10^{4}$ seconds at 400 stations. The stations are located on a grid of $20 \times 20$ (Fig. 1.a) with an inter-station distance of $80 \mathrm{~km}$. The data errors are assumed to be uncorrelated and normally distributed with a standard deviation of $12 \%$ for the apparent resistivities and 1.5 degrees for the phases. We avoid the so-called "inverse crime" by generating the data with a finer FE mesh than that used in the inversions. This also means that a perfect fit to data may not be achievable during the inversion.

\subsection{Example 1: Large-scale Lithospheric Structure}

\subsubsection{Model setup}

The area selected for the inversion is sub-divided into $14 \times 14$ columns (white squares in Fig. 1.b) of size $80 \times 80 \times 460 \mathrm{~km}$. The model parameters are the depths to the thermal lithosphere-asthenosphere boundary (LAB) of the 196 columns within the inversion area, i.e. there is one model parameter per column. Here, we identify the $\mathrm{LAB}$ with the depth to the $1250^{\circ} \mathrm{C}$ isotherm (cf. Afonso et al., 2016a). The goal is to retrieve the LAB depths of the model from noisy data.

The "true" model is shown in Figs. 1. In order to obtain the conductivity structure of the true model from LAB depths, we first compute the thermal structure by solving the steady-state heat transfer problem with Dirichlet boundary conditions at the surface $\left(T_{0}=10^{\circ} \mathrm{C}\right)$ and at each LAB depth $\left(T_{L A B}=1250^{\circ} \mathrm{C}\right)$. For simplicity, we assume a linear temperature gradient between the $\mathrm{LAB}$ and $410 \mathrm{~km}$ depth, where the temperature is fixed at $T_{410}=1550^{\circ} \mathrm{C}$. This gradient is extrapolated to the bottom of the numerical domain, located at $460 \mathrm{~km}$ depth. A pressure profile is also computed in each column using the following quadratic lithostatic-type approximation:

$$
P(z)=0.99 \times\left(4.4773 \times 10^{-3} z^{2}+3.2206 \times 10^{4} z-1.284278 \times 10^{8}\right)
$$

where $P$ is pressure in $P a$ and $z$ is depth in meters. As a further simplification, we assume a dry and homogeneous mantle composition with the following mineral modes: $0.520,0.202,9.798 e^{-2}$ and 


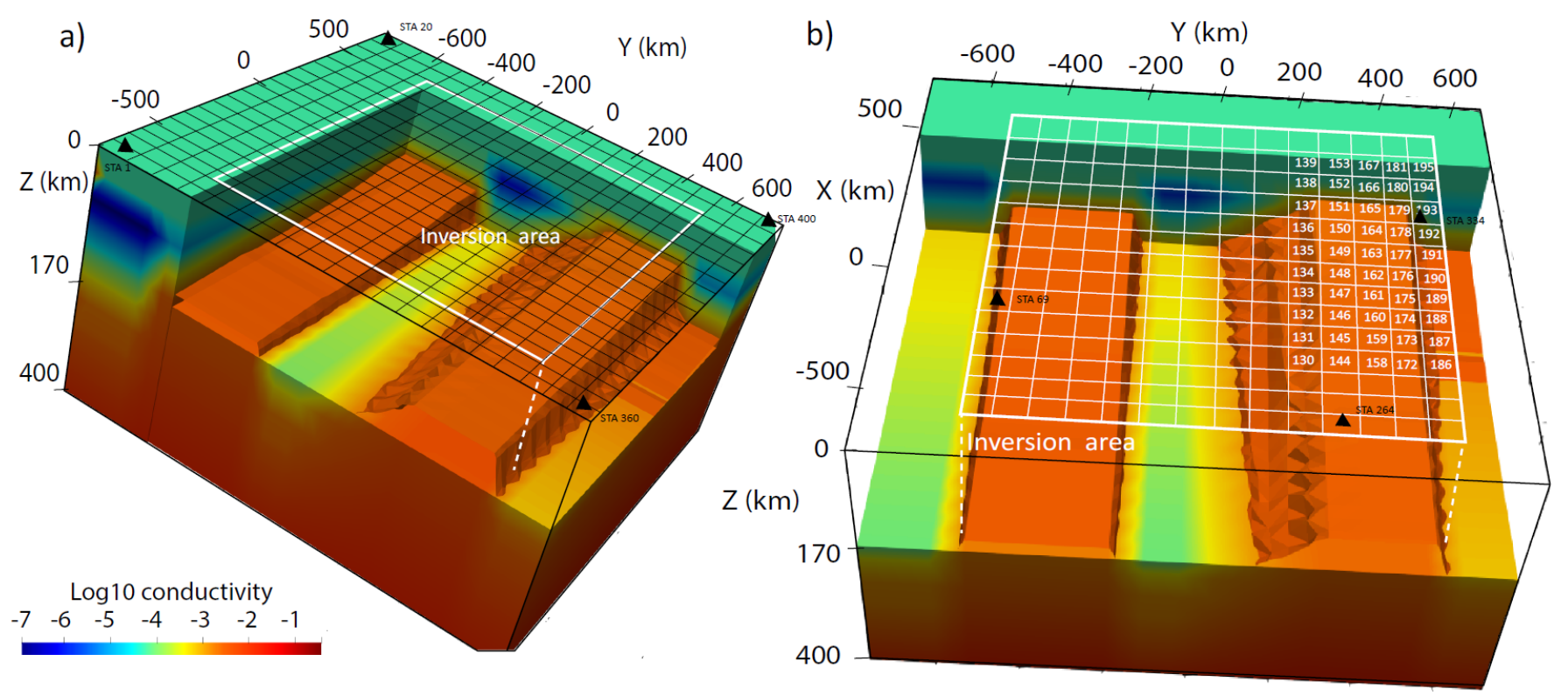

Figure 1. 3D rendering views of the true conductivity structure where the iso-surface of $-2.8 \log _{10} \mathrm{~S} / \mathrm{m}$ is plotted as a reference. The white rectangle indicates the region used for the inversion. Panel (a) illustrates the 20x20 station-grid in black and four of the 400 stations (black triangles). The model parameters are the depths to the LAB of 196 columns. Panel (b) displays the location of these columns (white small squares) and, in particular, the 50 columns shown in Fig. 5. The reader is referred to Section 6.2.1 for details on the parameterization.

0.179 vol\% for olivine, orthopyroxene, clinopyroxene and garnet, respectively. The electrical conductivity for each mineral phase is then obtained using Eq. A.3, with parameters specified in Table A1. Finally, the bulk electrical conductivity (i.e. that of the mineral aggregate or rock) of each FE cell in the mantle is obtained using the Hashin-Shtrikman averaging scheme (Hashin \& Shtrikman, 1962, 1963). The conductivity in the crust (Moho at $49 \mathrm{~km}$ depth) is constant and equal to $20,000 \Omega m$.

\subsubsection{Sampling strategy}

The priors for the LAB depths are uniform distributions defined in a range of $\pm 50 \mathrm{~km}$, centered on the true value of each column. The proposals are Gaussian distributions centered on the current sample with a standard deviation of $10 \mathrm{~km}$ (see details in Appendix B). The initial LAB model (i.e. starting point of the MCMC inversion) is defined by randomly choosing an LAB depth from the prior distribution of each parameter. At each step of the MCMC inversion, the algorithm randomly selects a column (metropolised independence sampler) and assigns an LAB depth from the proposal distribution. The new LAB is then used to re-compute the temperature and pressure of that column and update the conductivity model as explained in the previous section. 


\begin{tabular}{ccccc}
\hline & \multicolumn{2}{c}{ RMS conductivity $\left(\log _{10} S / m\right)$} & \multicolumn{2}{c}{ RMS LAB depth $(\mathrm{km})$} \\
\hline & Maximum a posteriori & Mean Model & Maximum a posteriori & Mean Model \\
\hline $\begin{array}{c}\text { RB+MCMC } \\
\text { ModEM }\end{array}$ & 0.19 & 0.15 & 21.20 & 17.01 \\
\hline
\end{tabular}

Table 1. Root-mean-square (rms) values of the mean and maximum a posteriori conductivity and LAB models with respect to the true model. The rms of the best conductivity model obtained with a ModEM deterministic inversion is also included.

\subsubsection{Inversion results}

We ran a total of 2,500,000 MCMC simulations using only 2 processors per frequency. Even with such modest computational resources, the inversion took $<30$ days (an average of 1.03 seconds per MCMC iteration). Since the high-fidelity solution for this model takes approximately 30 seconds, this represents a staggering time reduction of $\sim 97 \%$.

The mean conductivity model (i.e. mean values of the posterior PDFs) and the maximum a posteriori model are shown in Figs. 2 and 3, respectively. No lateral smoothing has been applied in either of these plots. These results demonstrate that our algorithm succeeded in retrieving a representative solution of the inverse problem. The root-mean-square (rms) values of both conductivity models with respect to the true model are listed in Table 1 where we have also included the rms of the LAB depths for both models (mean and maximum a posteriori) with respect to their true values.

The posterior PDFs of data for station 334 and the posterior PDFs of 50 model parameters are shown in Figs. 4 and Fig. 5, respectively. Additional posterior PDFs of data for other stations and the posterior PDFs of all model parameters can be found in Section 3 of the Supplementary Material. The results show that the great majority of observations are contained within one standard deviation of the posterior PDFs of the data. In the case of the LAB depths, the marginal posterior distributions have mean values that agree well with the true LAB depths within one standard deviation.

A closer examination of Fig. 5 reveals the well-known "compensation effect" or model equivalence in MT and EM methods (e.g Wait, 1962; Mallick \& Verma, 1979; Zhdanov \& Keller, 1994; Hoffmann \& Dietrich, 2004; Park \& Ostos, 2013; Harinarayana, 1999), by which incorrect model parameters can compensate each other and result in a combined MT signal that is close to that associated with the true solution. In the present example, the individual LAB depths within a cluster of adjacent columns can be relatively far from their true values, but their combined effect still produce a conductivity model that fits the data well. The inherent compensation effect in MT is exacerbated here by the simple parameterization and sampling strategy. For instance, we are assuming that the conductivity 
a)

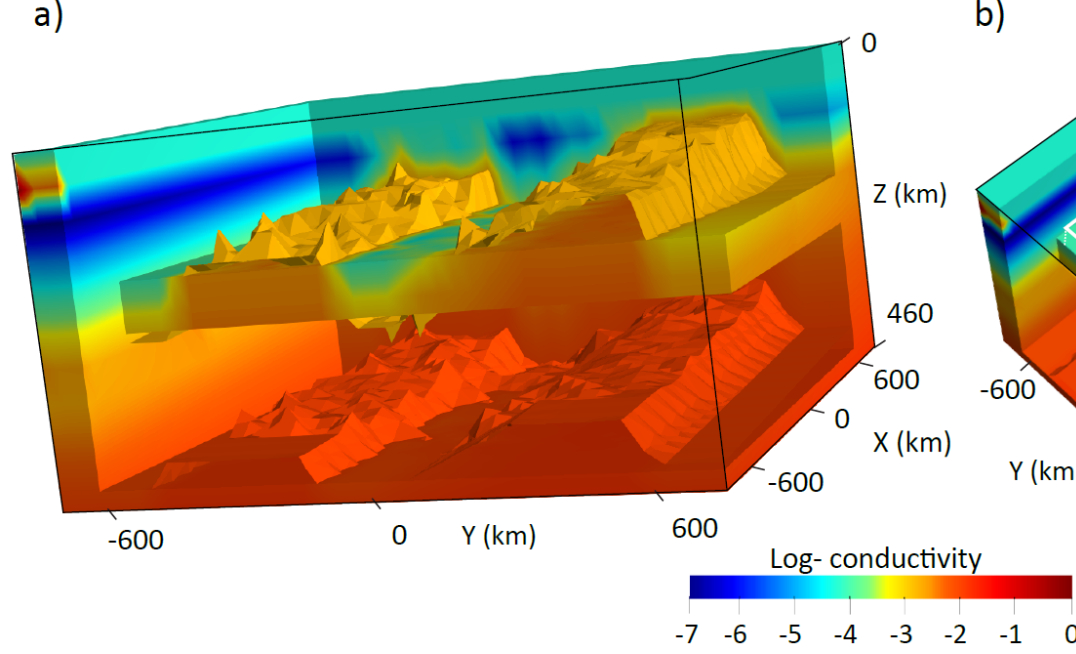

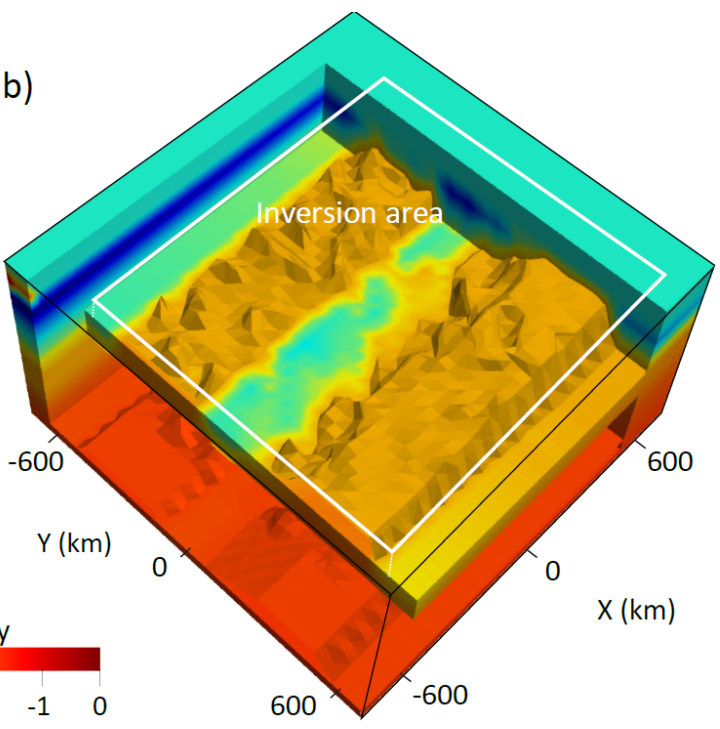

Figure 2. 3D rendering views of the mean conductivity structure obtained after 2,500,000 MCMC simulations.

The iso-surfaces of -2.8 and $-2 \log _{10}(\mathrm{~S} / \mathrm{m})$ are plotted as a reference. The white rectangle in $(\mathrm{b})$ indicates the region used for the inversion.
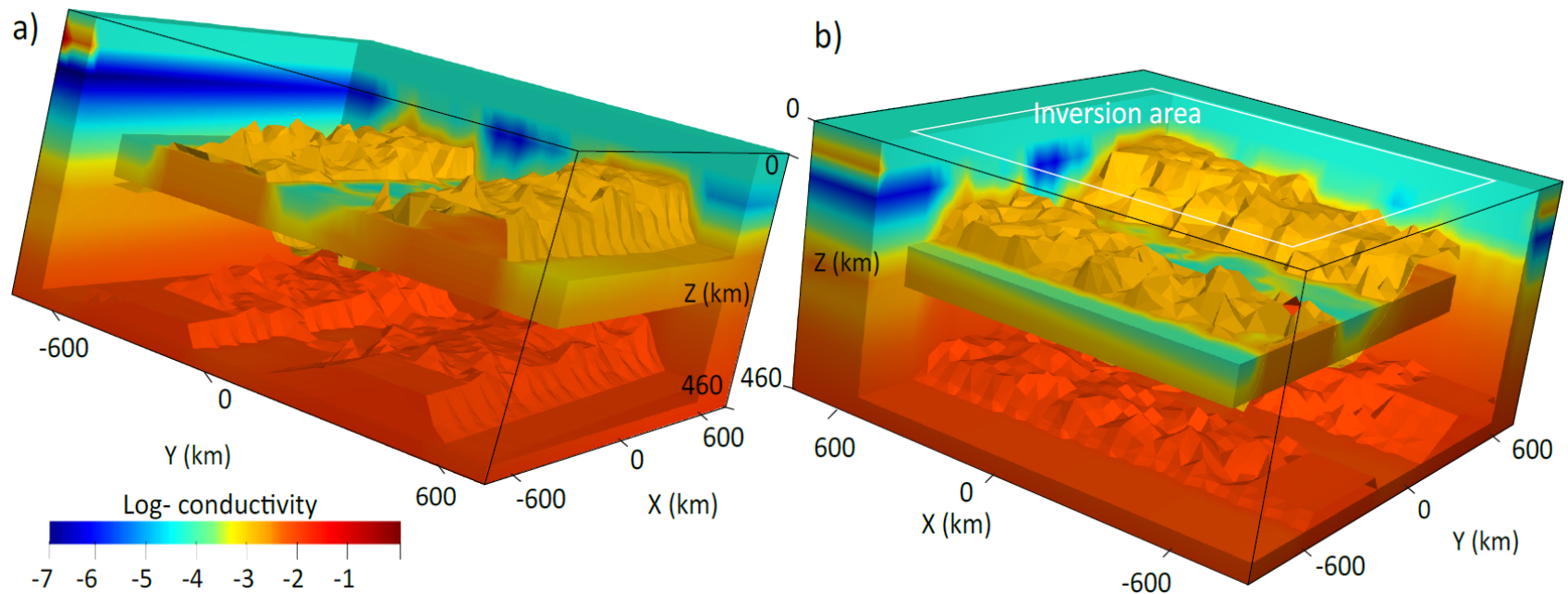

Figure 3. 3D rendering views of the maximum a posteriori (best-fitting) conductivity structure obtained after 2,500,000 MCMC simulations. The iso-surfaces of -2.8 and $-2 \log _{10} \mathrm{~S} / \mathrm{m}$ are plotted as a reference. The white rectangle in (b) indicates the region used for the inversion.

distribution is only controlled by the depth of the thermal LAB (Section 6.2.1), which means that decreasing the LAB depth in a column has the effect of increasing its average conductivity; the opposite is also true. Under this circumstances, a cluster of columns with LAB depths shallower and deeper than the true value for the cluster can produce a good fit to the data.

The effect of the sampling strategy can be understood as follows. Let us suppose that the current state of the MCMC chain contains a cluster of compensated columns with incorrect LAB values but 


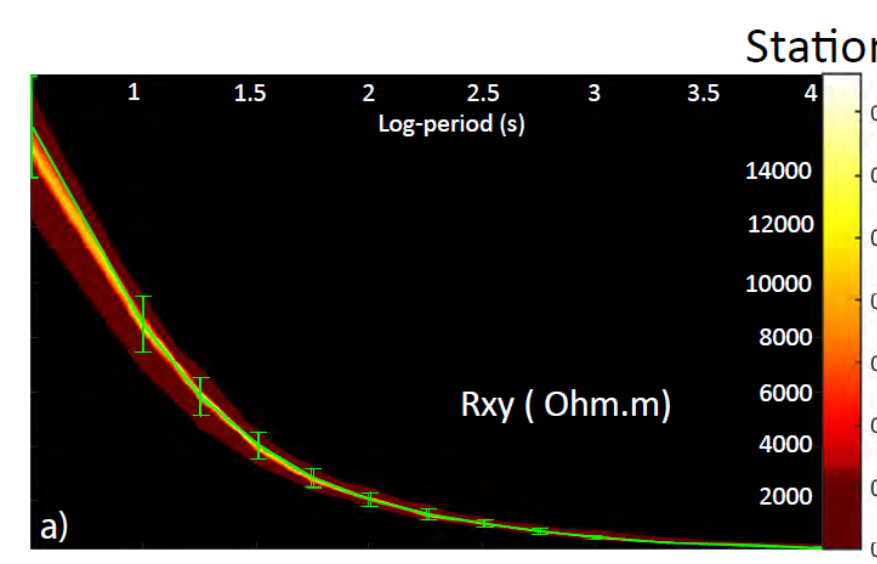

\section{4}
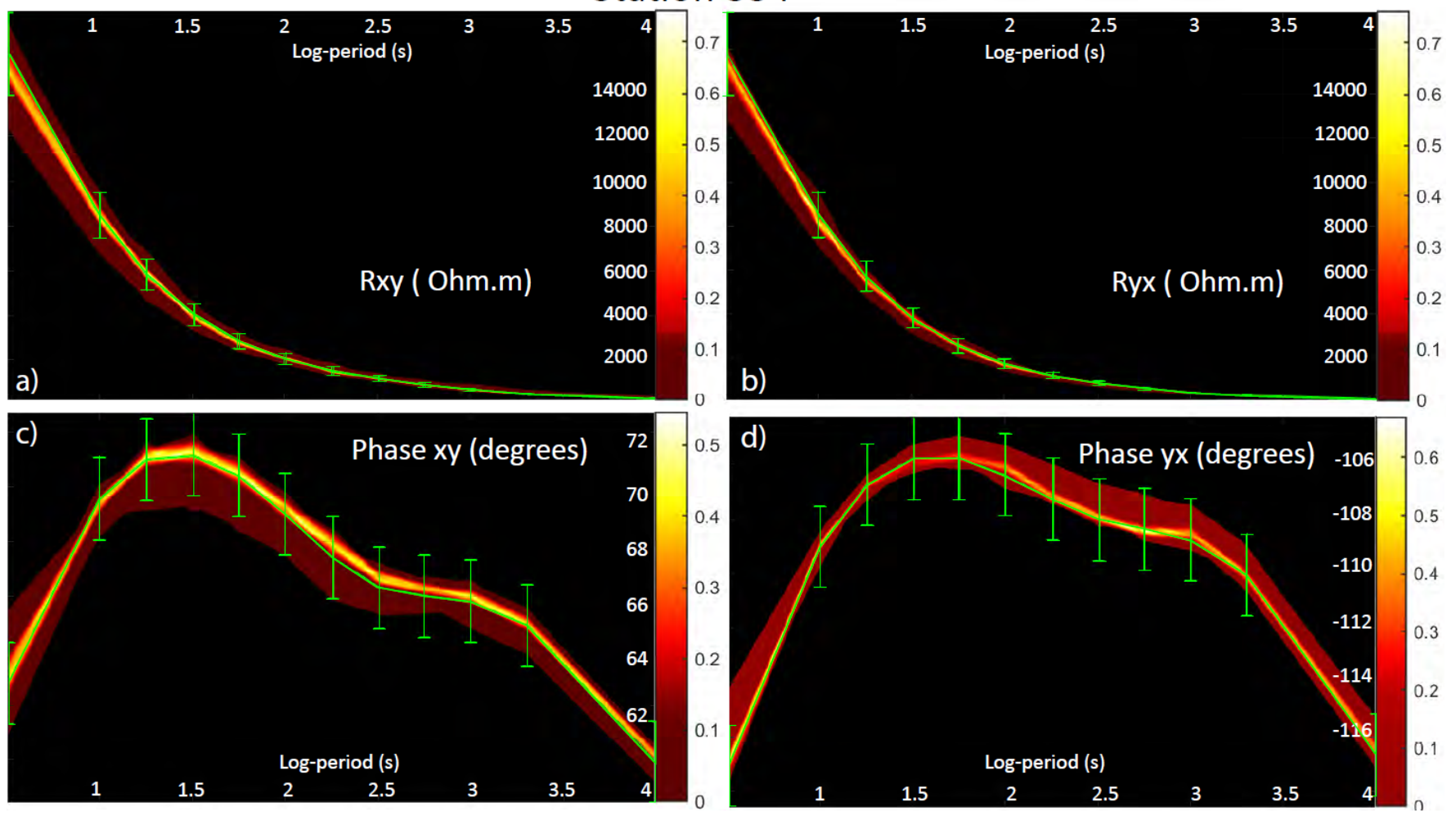

Figure 4. Posterior PDFs of data for station 334. Synthetic data and error bars are plotted in green. (a)-(b) Posterior PDFs of the off-diagonal apparent resistivity. (c)-(d) Posterior PDFs of the off-diagonal apparent phases.

with an acceptable fit to the data. Since each column is independent and we sample only one parameter (i.e. column) per MCMC iteration, if we propose a new LAB value for any of the columns in the cluster that is closer to the true value for that column, the combined MT signal will most likely produce a poorer misfit compared to the current compensated state. This is because although we are proposing a value closer to the true value for one column, it is the combined effect of the cluster that drives the misfit. In deterministic inversions, this problem is minimized by applying regularization terms. More appropriate paramaterizations and sampling strategies in the context of MCMC inversions are further discussed in Section 7.

The aforementioned compensation effect can be observed, for example, in adjacent columns 172 and 186 in Fig. 5, where the mean LAB depth is $\sim 15 \mathrm{~km}$ deeper than the true value for column 172 and $\sim 15 \mathrm{~km}$ shallower than the true value for column 186 . The same effect is also observed in columns 144 and 145 (with mean LAB depths deeper than their true value) and columns 158 and 159 (shallower mean LAB depths). For some parameters (178 for example), the compensation of 
a)

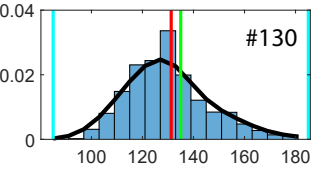

LAB depth $(\mathrm{km})$

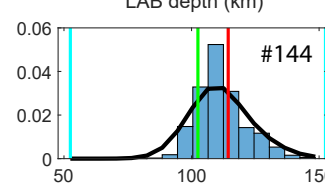

$\mathrm{LAB}$ depth $(\mathrm{km})$
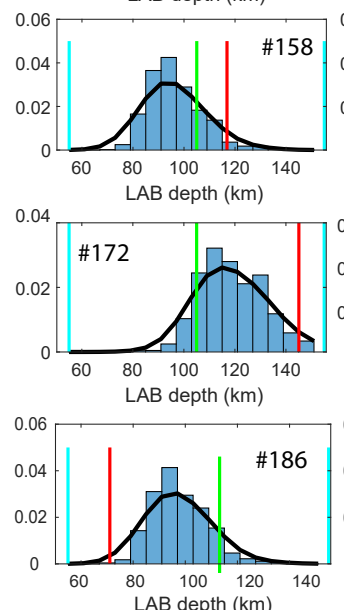

- LAB depth $(\mathrm{km})$

b)
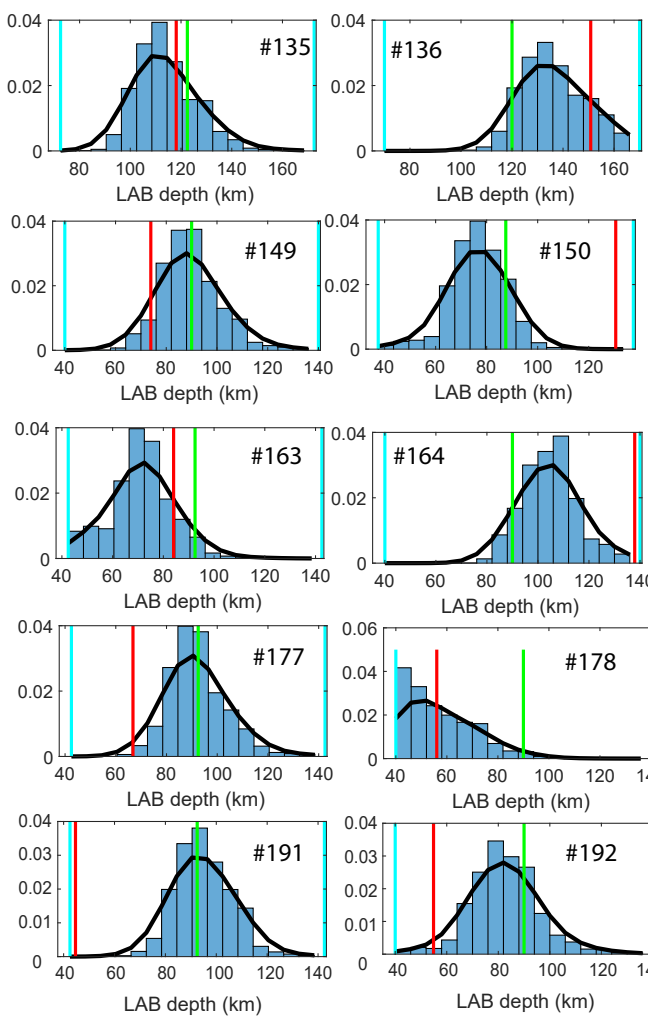
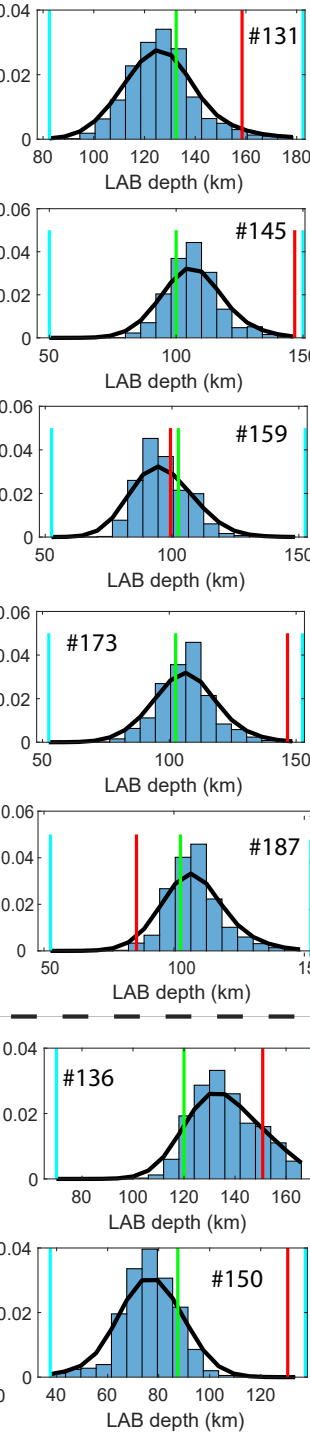

LAB depth $(\mathrm{km})$

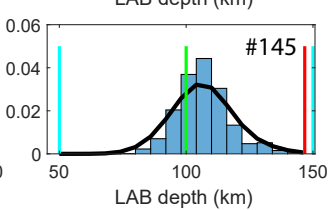

LAB depth $(\mathrm{km})$
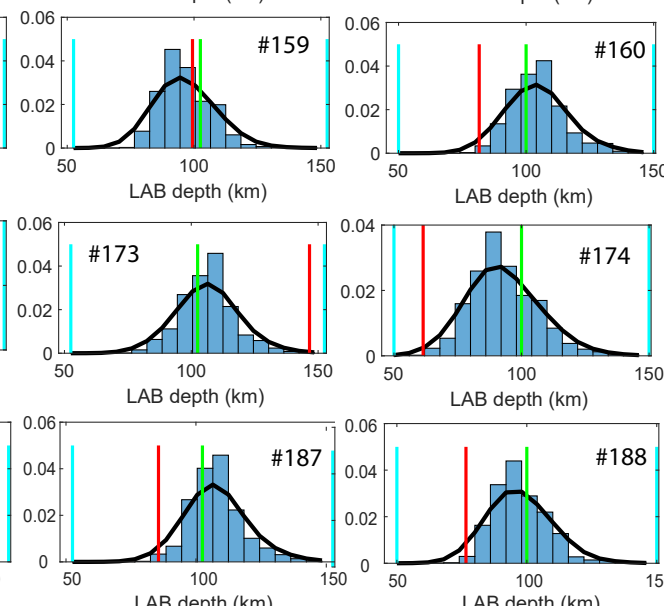

LAB depth $(\mathrm{km})$
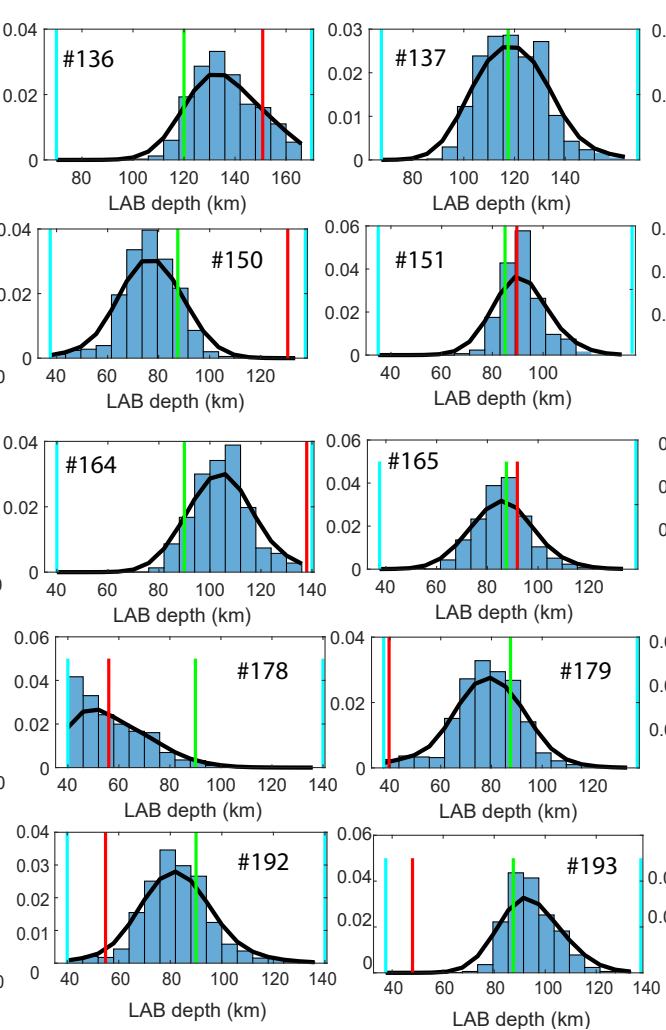
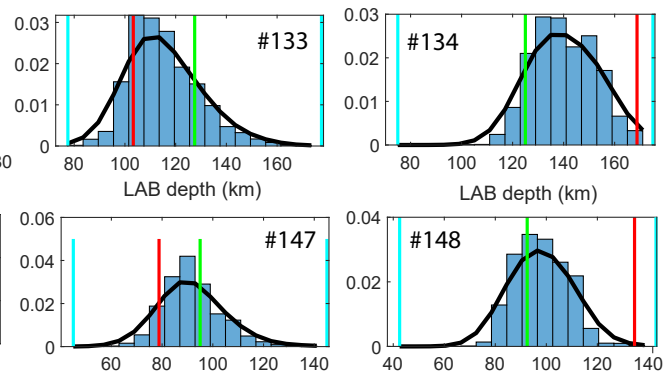

$\mathrm{LAB}$ depth $(\mathrm{km})$
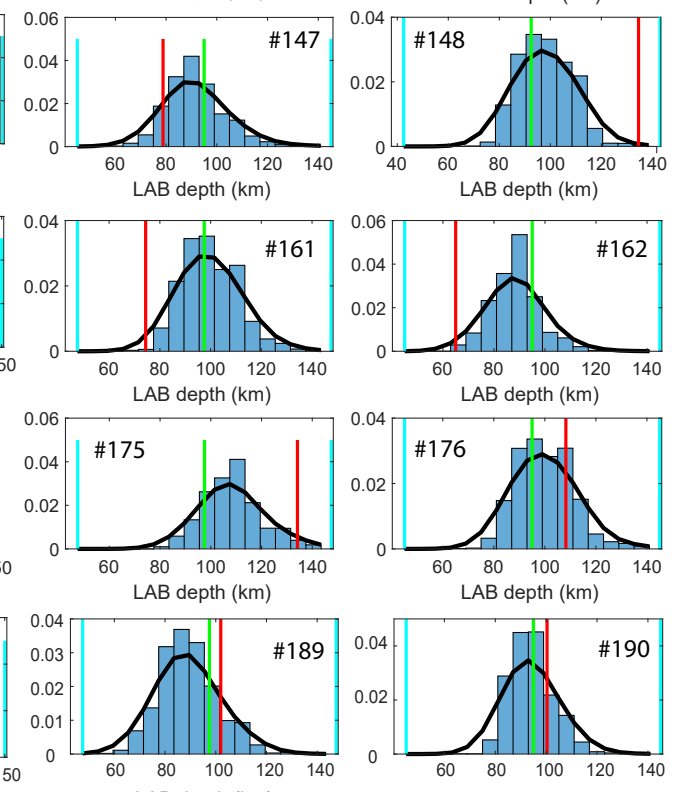
LAB depth $(\mathrm{km})$
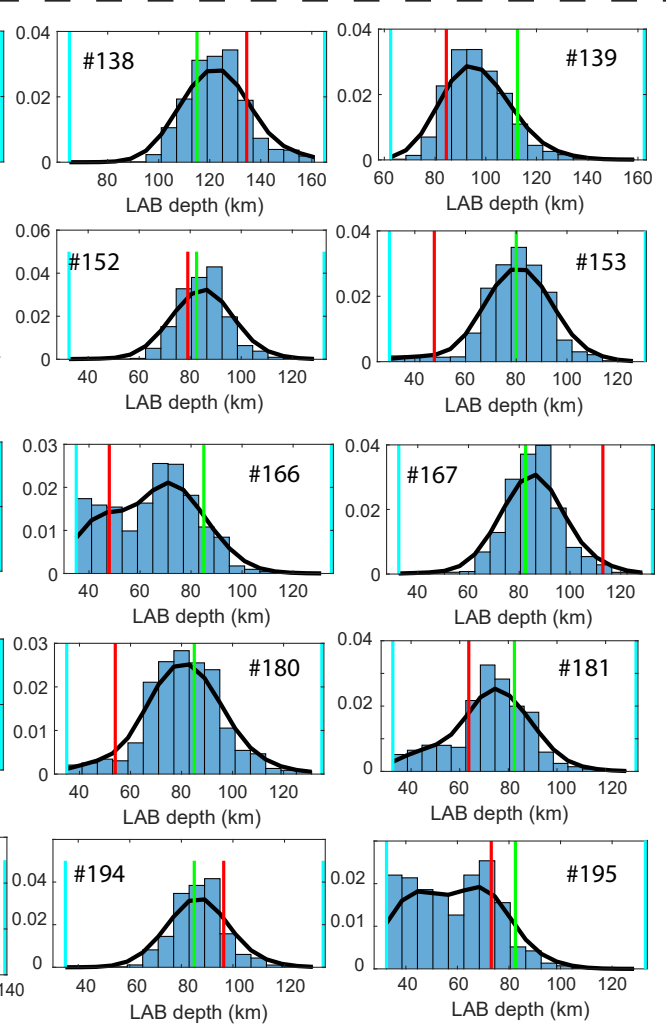

Figure 5. (a)-(b) Marginal posterior PDFs (blue bars) and best fitting distribution (black line) of 50 model parameters obtained after 2,500,000 RB+MCMC simulations. The real value, starting value and prior bounds of each parameter are shown in green, red, and light blue vertical lines, respectively. The numbers within each panel correspond to the columns highlighted in Fig. 1.b. 


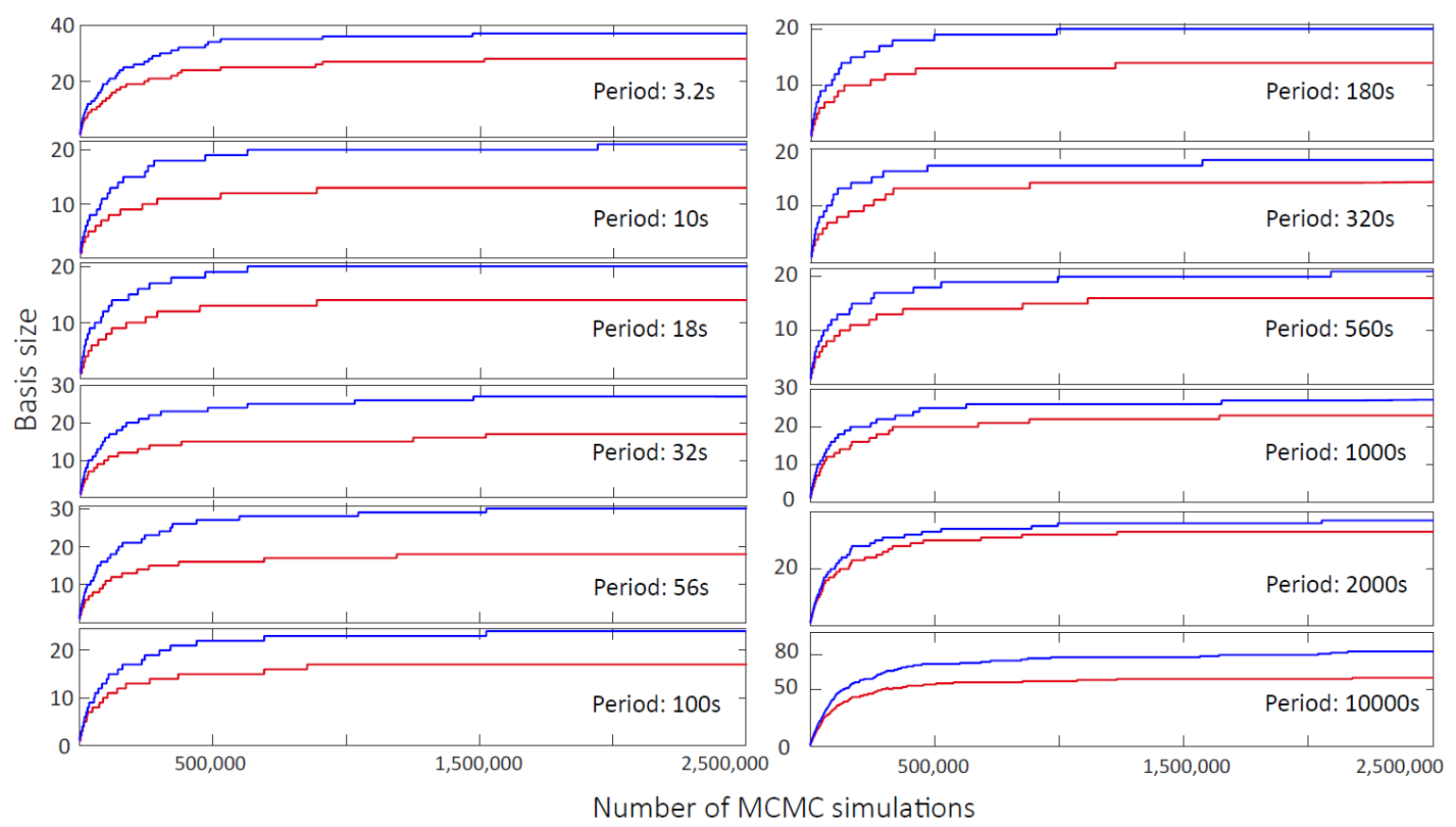

Figure 6. Basis size as a function of the MCMC simulations for different frequencies and field orientations $\left(S_{\perp}\right.$ mode in blue, and $S$ mode in red).

neighbouring parameters (columns 164,165, 177, 179, 191, 192 and 193) is such that they cannot be recovered well by the inversion given the range of data errors and the prescribed tolerance $\beta$.

The size of the basis computed per frequency and field orientations is shown in Fig. 6. A rapid increment in the basis size is observed during the burn-in stage (first 200,000 simulations) which also correlates with a rapid decrease in the overall misfit (Fig. 7). This increment is required to obtain representative solutions for the parameter space and, in particular, for the high-probability region. After this stage, the basis size reaches a saturation level or plateau for all frequencies, i.e the reduced basis space is able to reproduce any solution within the regions of high probability. At this point, the adaptation of the RB space could be stopped without compromising subsequent solutions provided that the chain has indeed reached stationarity.

For comparison, we include the results of a ModEM (Kelbert et al., 2014) deterministic inver-

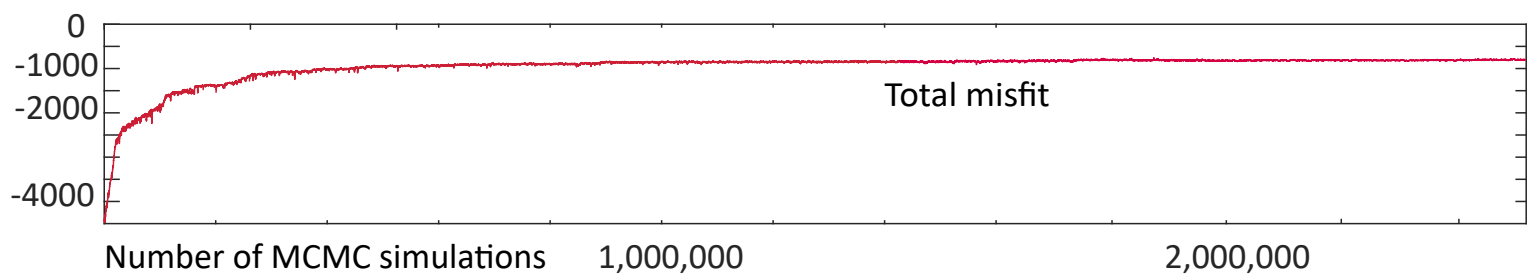

Figure 7. Data misfit for each of the 2,500,000 RB+MCMC simulations. 


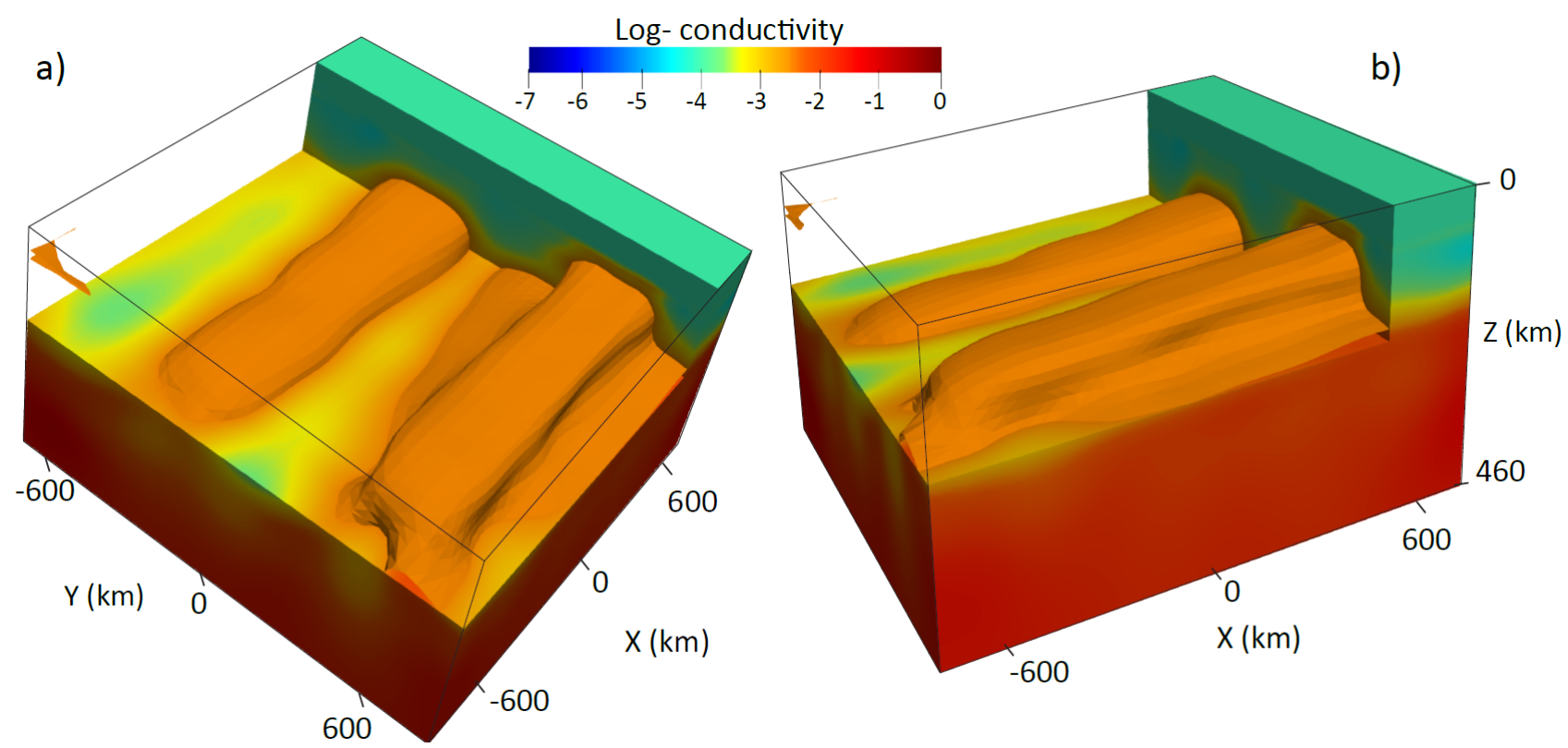

Figure 8. (a)-(b) 3D rendering views of the best conductivity model obtained with a ModEM deterministic inversion using a horizontal and vertical smoothing factor of 0.5 and 0.2 , respectively. The iso-surface of -2.8 $\log _{10}(\mathrm{~S} / \mathrm{m})$ is plotted as a reference.

sion using the same synthetic data. Fig. 8 shows the best model obtained after 42 iterations using a vertical and horizontal smoothing factor of 0.2 and 0.5 , respectively. The inversion took 6 hs using 30 processors and the final data rms was 1.04. The rms value of the ModEM model with respect to the true conductivity distribution is also included in Table 1. One of the main reasons for the larger rms in the ModEM inversion is that lateral smoothing had to be imposed to stabilize the inversion (a typical requirement in determinisitc inversions). This precluded the algorithm from considering sharp discontinuities in the solution. Another contribution to the rms is the high-resistivity regions in the mantle, which could not be recovered in this example.

\subsection{Example 2: Large-scale Lithospheric Structure with Conductivity Anomalies}

\subsubsection{Model setup}

The true conductivity model (Fig. 9) includes the large-scale lithospheric model of Section 6.2 as a background plus three additional and localized conductive anomalies. The conductivity structure is now parameterized by 1782 nodes (black dots in Fig. 9a) sparsely located within the inversion volume. The conductivity value of each FE cell is obtained by interpolation of the nodes' values using a quadratic Shepard method for trivariate interpolation of scattered 3D data (Renka, 1988b,a). The goal here is to recover the conductivity structure from noisy MT measurements. 


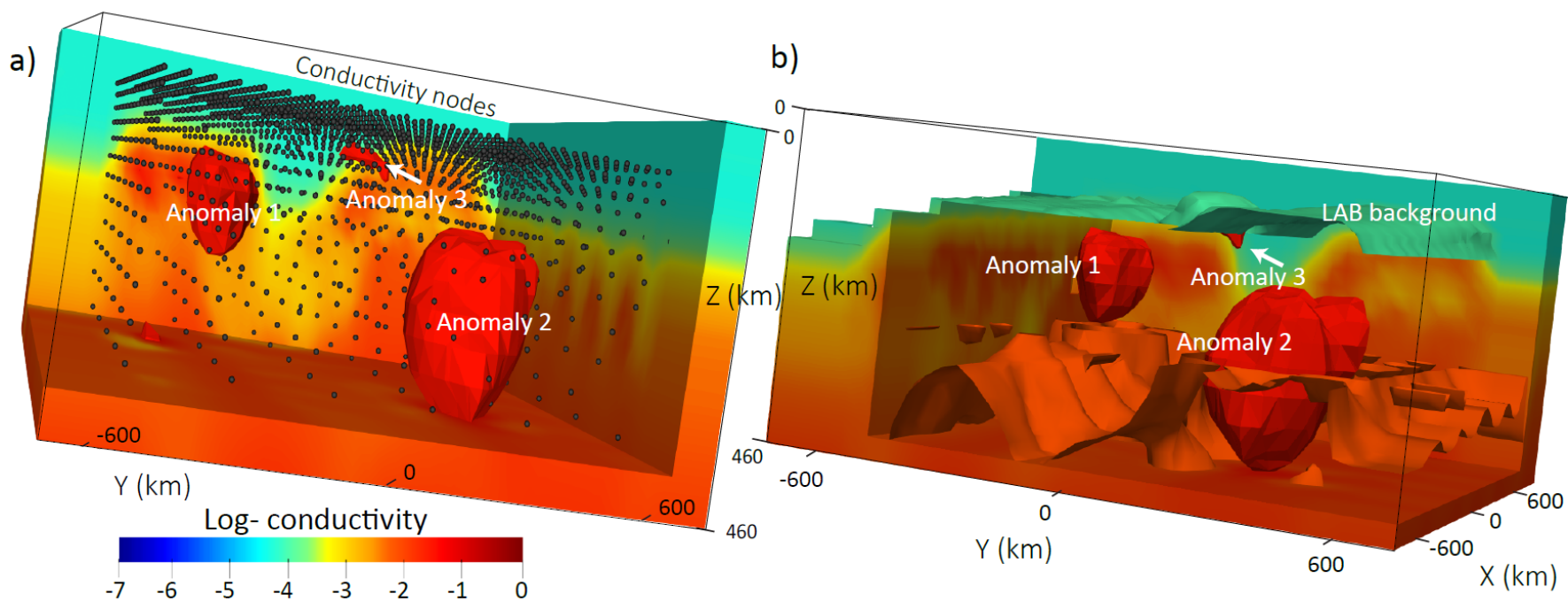

Figure 9. 3D rendering views of the true conductivity structure after interpolation of the 1782 node-parameters. Conductive anomalies are highlighted in both (a) and (b) panels. Black dots in (a) indicate the position of the node-parameters within the inversion volume. Panel (a) shows the iso-surface corresponding to $-1.5 \log _{10}(\mathrm{~S} / \mathrm{m})$, whereas iso-surfaces of $-2.15,-1.5$ and $-4 \log _{10}(\mathrm{~S} / \mathrm{m})$ are shown in (b).

In order to define the location of the conductivity nodes, we first sub-divide the domain into 14 horizontal layers; the vertical location of the nodes correspond to the mid-points of each layer (horizontal node-layers in Fig. 9.a). In this example, these layers correlate with the horizontal layers given by the FE mesh. Since bodies with dimensions smaller than the EM skin depth cannot be resolved by the data, the horizontal distance between nodes within each layer is chosen relative to the skin depth for the range of periods and apparent resistivities shown in the observed data. We discuss alternative options for dynamic parameterizations in Section 7.

\subsubsection{Sampling strategy}

The starting model (Fig. 10) is defined by randomly selecting a conductivity value for all nodes from their prior distributions. Since the model parameters' values can span several orders of magnitude, we define Jeffreys' prior distributions (see Appendix B) in a range of $\pm 2 \log _{10}(S / m)$ centered on the true value of each node. During the burn-in stage, the algorithm randomly chooses one node $i$ at a time (with all nodes having the same probability of being chosen) and assigns a conductivity value from its proposal distribution. The proposals are defined as log-normal distributions (Eq. B.4) centered on the node $m_{t-1}^{i}$ with standard deviations of $0.5 \log _{10}(S / m)$. For every new trial $m_{t}^{i}$, the full $3 \mathrm{D}$ conductivity model is updated via interpolation (using a quadratic Shepard method, Section 6.3.1).

After the burn-in stage, we use an Adaptive Metropolis algorithm (Haario et al., 2001) to compute 
Figure 10. 3D rendering views of the initial conductivity structure. The iso-surfaces of -4 and $-1.5 \log _{10}(\mathrm{~S} / \mathrm{m})$ are plotted as a reference in both (a) and (b) panels.

and update layer-wise proposal distributions using the history and correlations of the MCMC chain (see details in Appendix B1). At each MCMC step, a metropolised independence sampler randomly selects a layer $l$ and the adapted proposal (Eq. B.5) is used to propose a new sample $\mathbf{m}^{l}$ (i.e. conductivity values for all the nodes of layer $l$ ). This sampling approach results in significant gains in efficiency and makes better use of the natural correlations inferred from the data.

\subsubsection{Inversion results}

We ran a total of 2,500,000 MCMC simulations for 11 frequencies using only 2 processors per frequency. The tolerances used were $\beta=0.07$ for the first 150,000 steps and $\beta=0.05$ for the remaining of the simulation. The SVD factorization was performed every 50 new bases with an energy cutoff of 0.997 . The first adaptation of the proposals for all node-layers was done at step 200,000 and we continue to adapt them every 100,000 simulations. Again, even with modest computational resources, the inversion took $<40$ days with an average of 2.88 seconds for each of the first 300,000 simulations (burn-in and enrichment process) and 1.58 seconds for the rest of the simulations. This corresponds to a time reduction of $\sim 95 \%$ for each forward computation after the enrichment process. It is important to note here that most of the computational time (besides the high-fidelity solutions) is now taken by the interpolation steps rather than by the surrogate itself. As discussed in Section 7 the inversion can be made significantly more efficient by changing the interpolation algorithm.

The mean conductivity model (i.e. mean values of the posterior PDFs) is shown in Figs. 11. It is clear that our RB+MCMC algorithm and the present parameterization result in a good approximation of the reference model, including the location and volumes of the conductive anomalies. Examples of the posterior PDFs of the data for two stations are shown in Figs. 12 and 13; the marginal posterior 


\section{M.C. Manassero et al.}

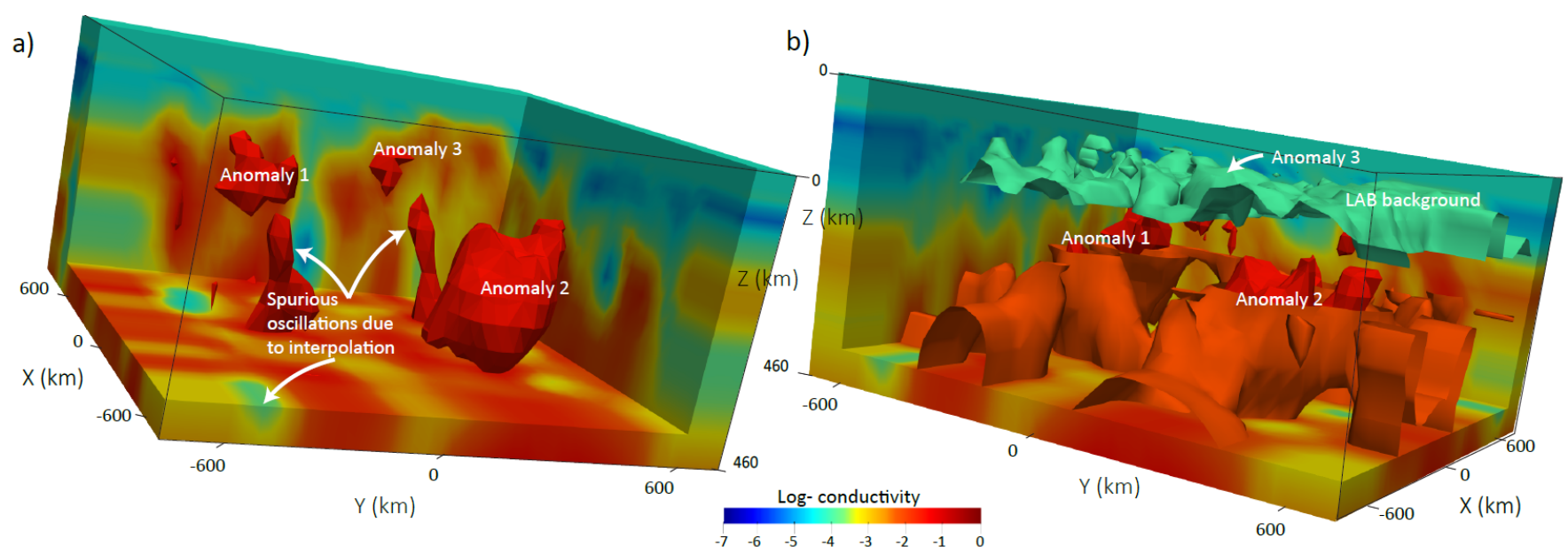

Figure 11. 3D rendering views of the conductivity structure obtained with the mean values of the posterior PDFs. Conductive anomalies are indicated in both (a) and (b) panels. Panel (a) shows the iso-surface of -1.5 $\log _{10}(S / m)$, whereas the iso-surfaces corresponding to $-2.15,-1.5$ and $-4 \log _{10}(S / m)$ are shown in (b).

PDFs of 50 of the 1782 model parameters (nodes) are shown in Fig. 14. Additional posterior PDFs can be found in Section 4 of the Supplementary Material.

As in the previous example, we also observe a bias of the models with respect to the true model. This is primarily due to the compensation effect described in Section 6.2.3, which is aggravated here

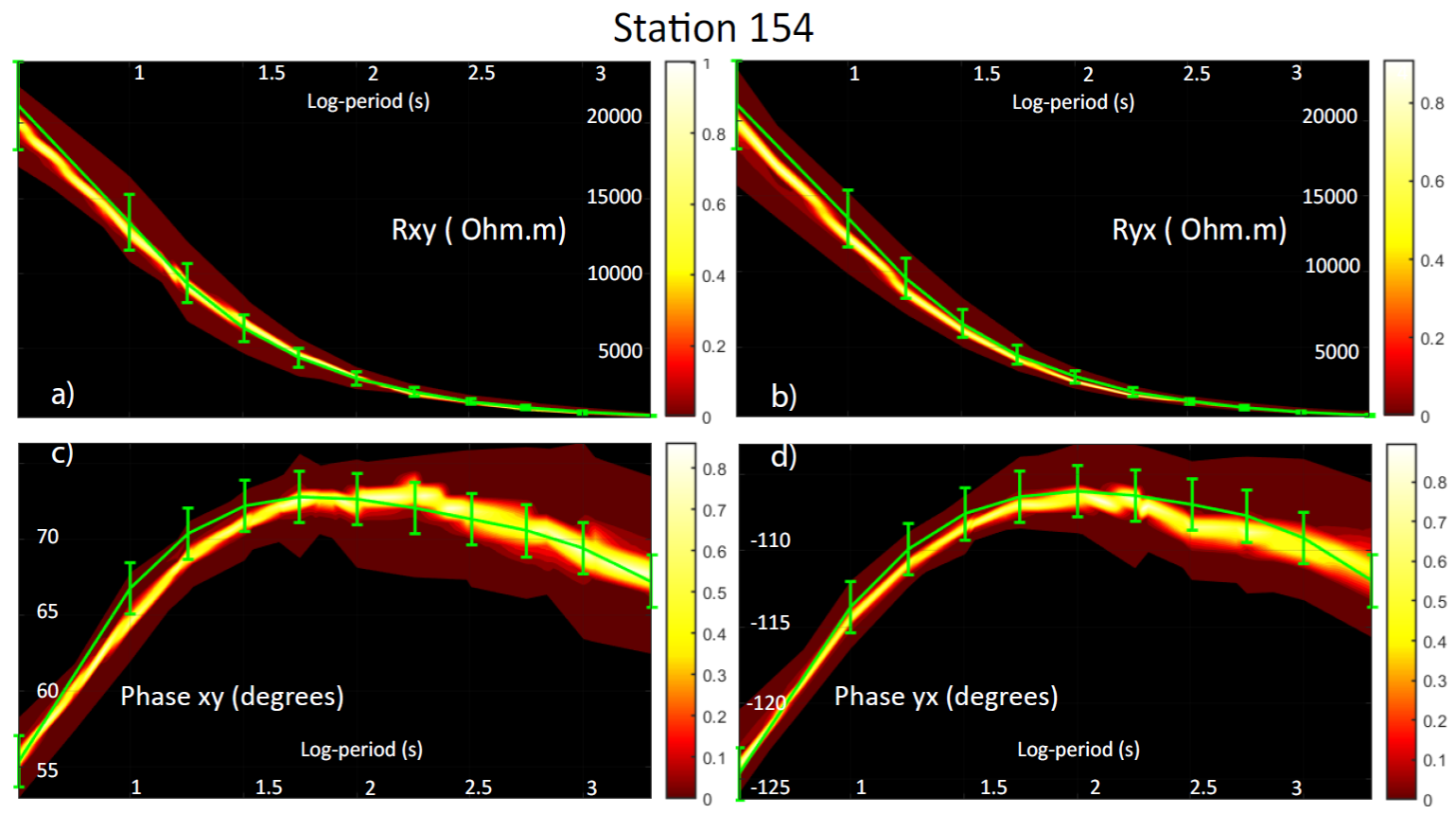

Figure 12. Posterior PDFs of data for station 154. Synthetic data and error bars are plotted in green. (a)-(b) Posterior PDFs of the off-diagonal apparent resistivity. (c)-(d) Posterior PDFs of the off-diagonal apparent phases. 


\section{Station 340}
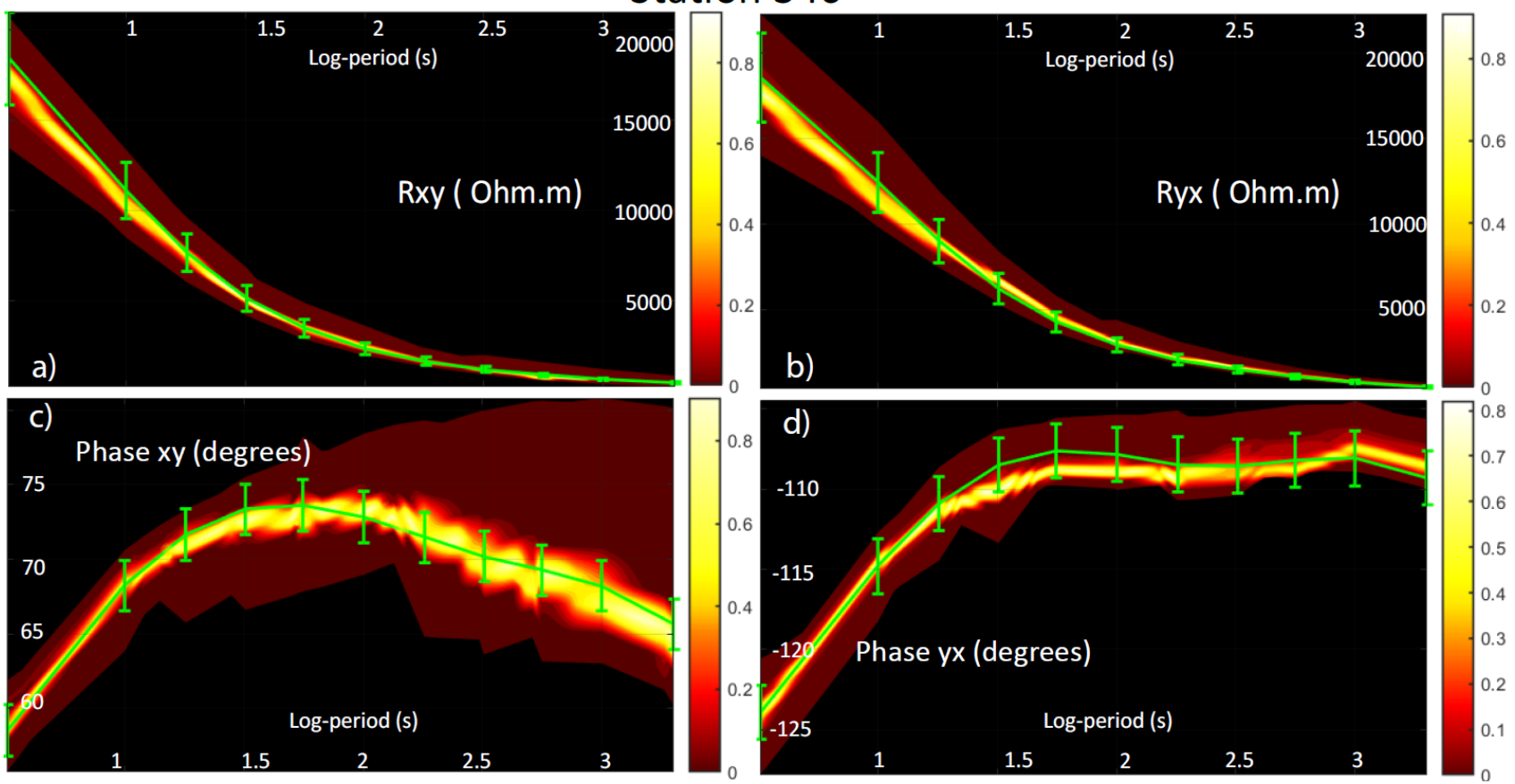

Figure 13. Posterior PDFs of data for station 340. Synthetic data and error bars are plotted in green. (a)-(b) Posterior PDFs of the off-diagonal apparent resistivity. (c)-(d) Posterior PDFs of the off-diagonal apparent phases.

by the introduction of spurious oscillations produced by the Shepard interpolation (shown in Fig. 11.a). In other words, since the Shepard interpolation introduces anomalous features in regions without nodes, the conductivity nodes that are located close to these spurious anomalies will most likely take incorrect (biased) conductivity values in order to produce models with better fits to the data. This bias can be further explained with a possible over-parameterization of the computational domain. In overparameterized and under-regularized regions, models are expected to exhibit local variability around the true structure and still offer equally good fits to the data. We also note that the mean values of the PDFs are more likely to be biased towards resistive values. This is due to the low relative sensitivity of MT data to resistive structures (e.g. Berdichevsky \& Dmitriev, 2010; Varentsov et al., 2013; Park \& Ostos, 2013). This effect is well-known and it has been observed in other works (e.g. Brodie \& Jiang, 2018; Rosas-Carbajal et al., 2013).

The number of basis vectors computed per frequency and field orientation is shown in Fig. 15. It can be seen that the number of bases rapidly increases during the first 120,000 steps while the inversion tries to find the regions of high probability. A second rapid increase is seen after step 150,000, which corresponds with a reduction in the tolerance of the surrogate (vertical black line in Fig.15). The basis size reaches a saturation level for all frequencies after $\sim 200,000$ steps. For the long periods, we 
M.C. Manassero et al.

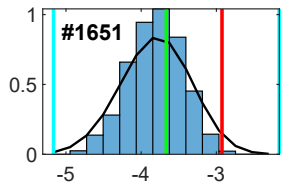

$\stackrel{-5}{\log 10^{-4}}$ conductivity

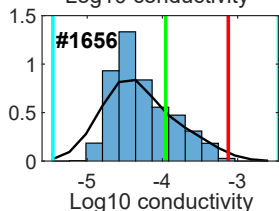

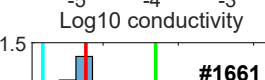

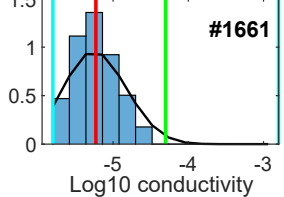

$\log ^{-5} 10$ conductivity $^{-3}$

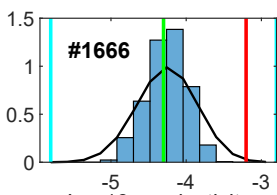

$\log ^{-5} 10$ conductivity $^{-3}$

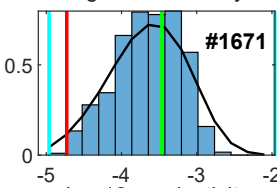

${ }^{-5} \log ^{-4} 0^{-3}$ conductivity $^{-2}$
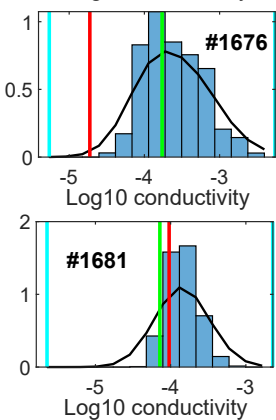

1. \#1686

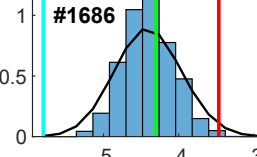

${ }^{-5}$ conductivity $^{-3}$

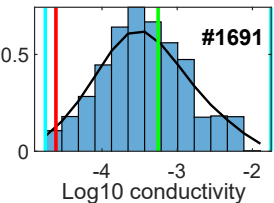

$\log ^{-4} 10$ conductivity $^{-2}$

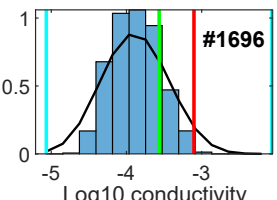

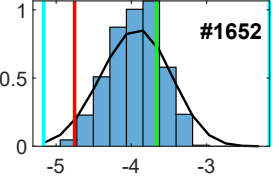

$\stackrel{-5}{\log 10^{-4}}$ conductivity $^{-3}$
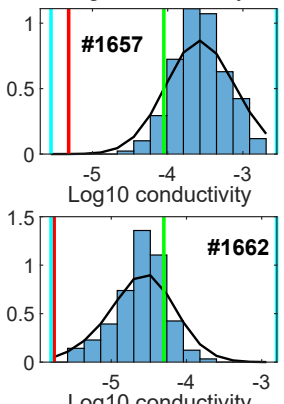

Log10 conductivity

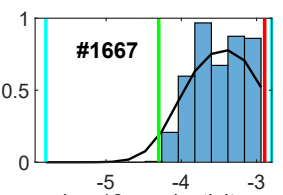

$\log ^{-5} 10$ conductivity $^{-3}$
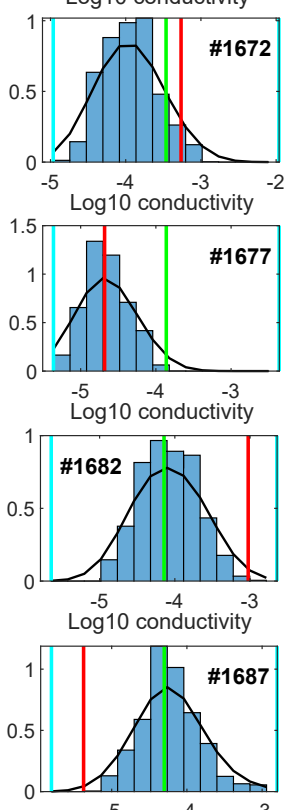

$\log ^{-5} 10$ conductivity $^{-3}$
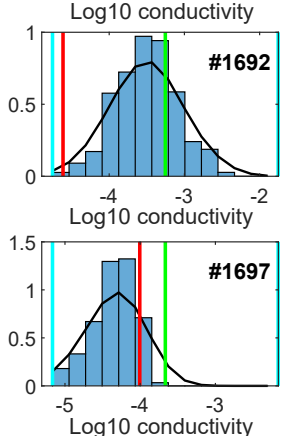
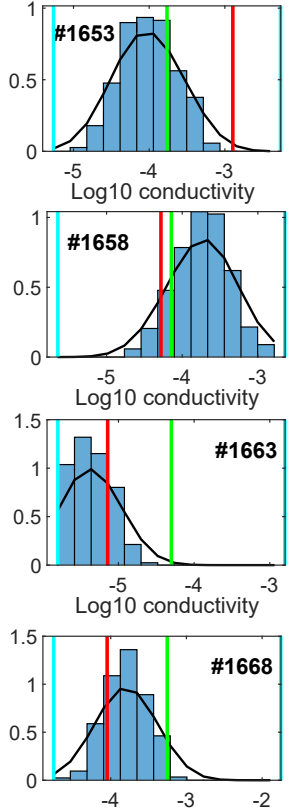

$\log ^{-4} 10$ conductivity $^{-2}$

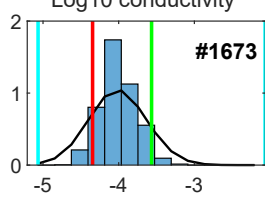

${ }^{-5} \log 10^{-4}$ conductivity
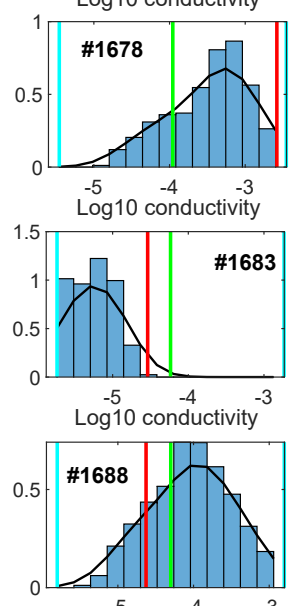

$\log ^{-5} 10$ conductivity $^{-3}$
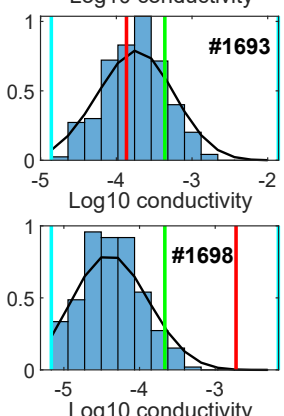
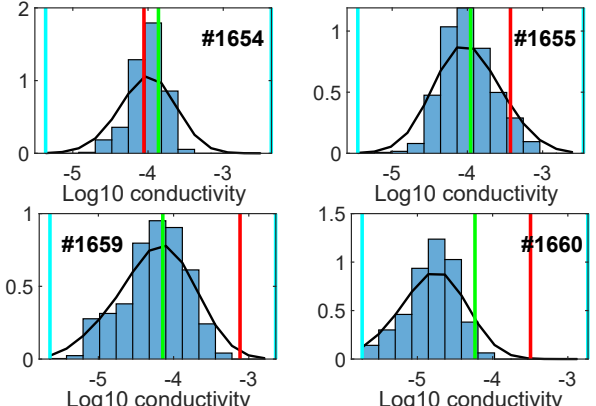

$\log ^{-5} 10$ conductivity $^{-3}$
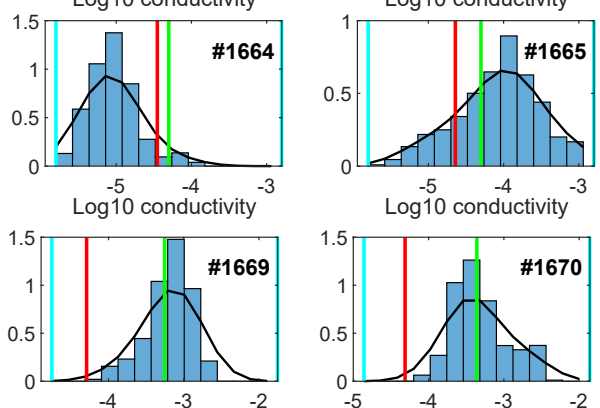

$\log ^{-4} 10$ conductivity $^{-2}$

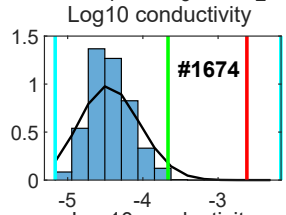

${ }^{-5} \log 10^{-4}$ conductivity
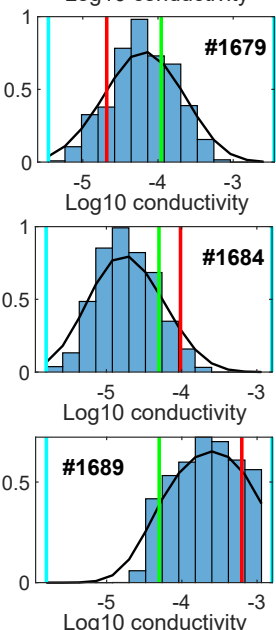

$\log ^{-5} 10$ conductivity $^{-3}$
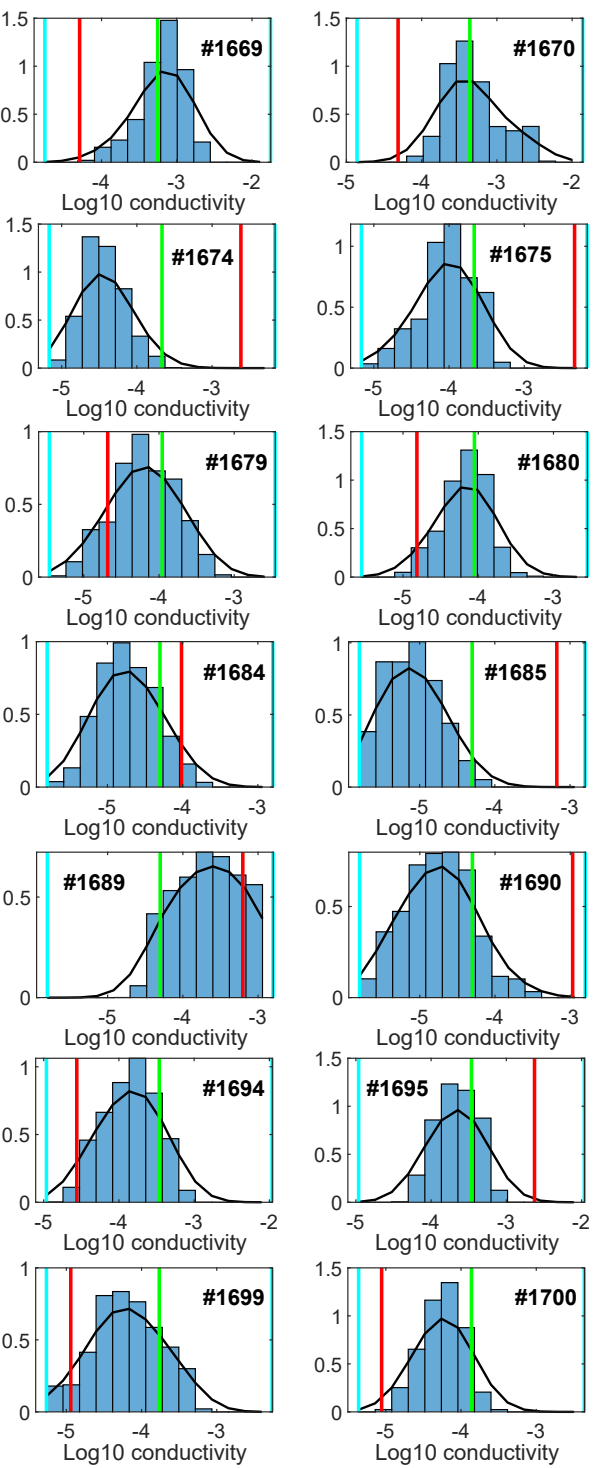

Figure 14. Marginal posterior PDFs (blue bars) and best fitting distributions (black lines) for 50 of the 1782 node-parameters (corresponding to the same node-layer) obtained after 2,500,000 RB+MCMC simulations. The real value, starting value and bounds of each parameter are shown in green, red, and light blue vertical lines, respectively. The reader is referred to the main text for details on the node-layer parameterization. 


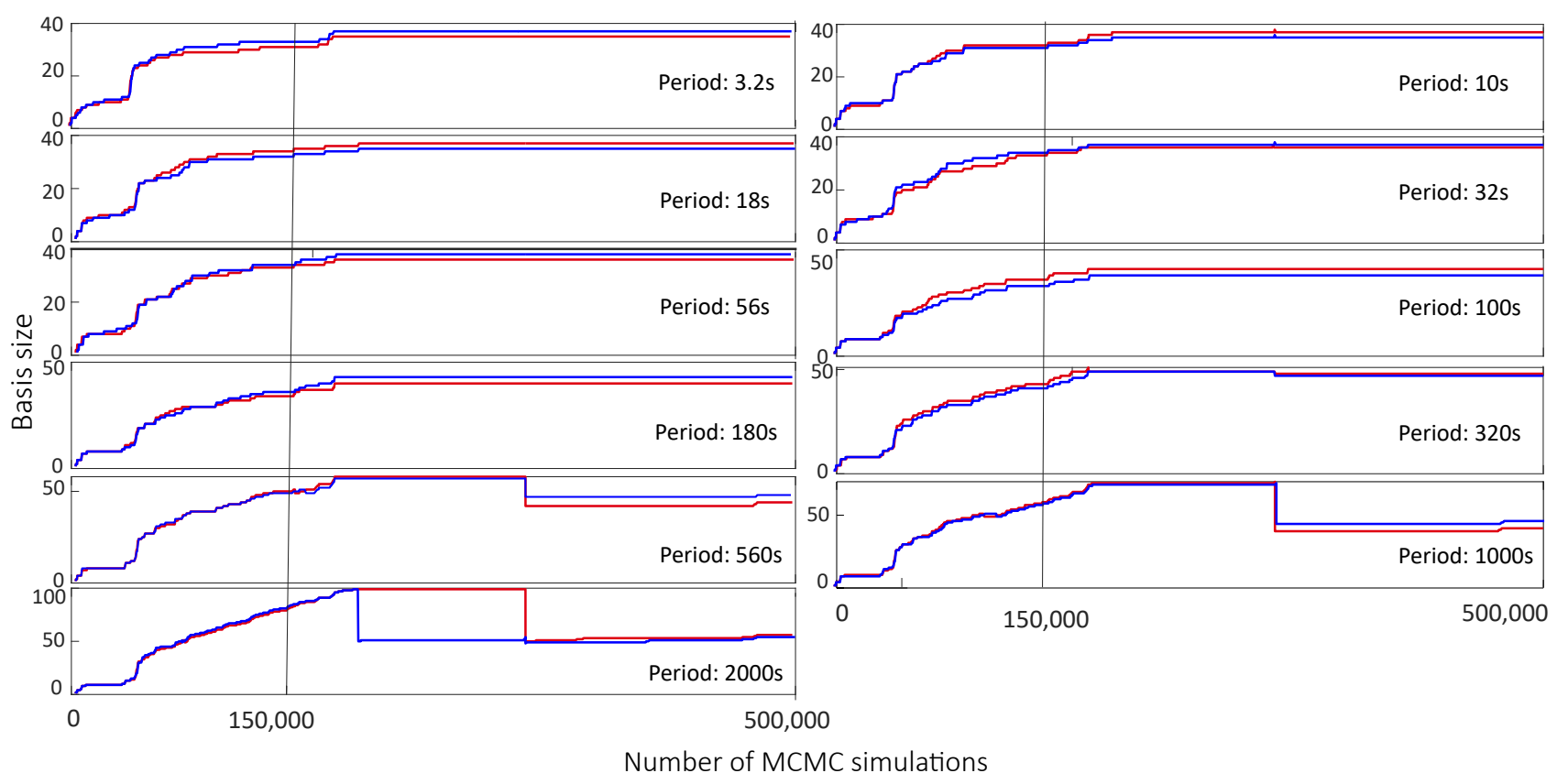

Figure 15. Basis size as a function of the MCMC simulations for different frequencies and field orientations $\left(S_{\perp}\right.$ mode in blue, and $S$ mode in red).

see that the SVD factorization at around step 300,000 significantly decreased the size of the basis. Subsequent basis vector computation and/or SVD factorizations do not change the general pattern and therefore we only show the bases up to the simulation 500,000.

The data misfit for the 2,500,000 simulations is shown in Fig. 16.a. As shown in Figs .16.b and 16.c, the enrichment of the basis space correlates with jumps in the total misfit. This is related to the fact that when a high-fidelity solution is computed, the misfit of the previous sample is also recomputed with the enriched space (see Section 5.1). As this new misfit value is "more accurate" and usually lower (worse) than the one computed before the enrichment, jumps occur in the general decreasing tendency of the misfit (Figs .16.b and 16.c). Note that these jumps are also observed in Fig. 7 albeit smaller in amplitude compared to those in Figs. 16. These jumps are less frequent as the basis reaches a saturation level (after 200,000 simulations), which corresponds to a quasi-plateau in the misfit.

\section{DISCUSSION}

In this work, we have deliberately chosen to work with the simplest parameterizations and sampling strategies in order to demonstrate the viability and performance of our approach under adverse circumstances. There are, however, a large number of possible improvements that can enhance the efficiency of our algorithm (e.g. the efficiency of the MCMC algorithms, the availability of computational re- 


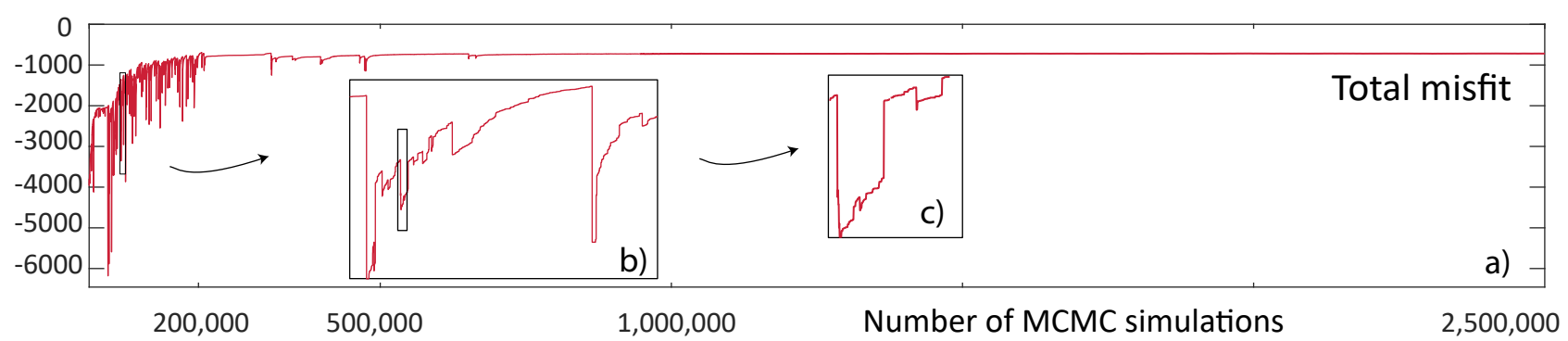

Figure 16. (a) Data misfit for each of the 2,500,000 simulations. Panels (b) and (c) show the jumps in the misfit when a new basis vector is computed. The reader is referred to the main text for an explanation of this behaviour in the misfit.

sources, the parameterization of the model). In the following sections we briefly discuss some of these potential improvements as well as some remaining challenges.

\subsection{Parameterization of the Conductivity Structure}

An important concept that we emphasize here is that the conductivity distribution in the lithosphere can, in principle, be treated as the superposition of two contributions: a background conductivity related to the long-wavelength physical state, and an anomalous distribution associated with the presence of smaller features (fluid pathways, melt-rich regions, hydrogen content, etc). Assuming that the conductivity of rocks were primarily controlled by the thermal structure of the lithosphere, the inversion performed in Section 6.2 demonstrated that the long-wavelength (i.e. background) conductivity structure can be recovered satisfactorily using an LAB-based parameterization. Subsequently, we introduced a more general, node-based, parameterization in order to include smaller-scale conductivity anomalies within the background (Section 6.3). While in principle this parameterization is capable of approximating any conductivity structure and allows for considerable model variance, a Shepard-type interpolation is slow (i.e. computationally inefficient) and typically introduces spurious oscillations that distort or mask the true conductivity structure (see Fig. 11.a). The latter effect is exacerbated when the number and location of the nodes used in the discretization are not optimal to allow an accurate representation of the true anomalies. This is likely the case in most practical scenarios, as it is generally impossible to know a priori the true variability of the conductivity in the subsurface (in practice, preliminary or nested inversions can be used for this purpose). Although it may seem reasonable at first glance to simply increase the number of nodes (and thus allow for more model variance), this can easily result in an over-parameterization of the model, which can seriously compromise the convergence of the MCMC algorithm (and any inversion scheme) and increase the computational time of the interpolations. 
A number of strategies can be pursued to ameliorate this problem. One possibility is to make use of well-known parameter model reduction techniques (e.g. Lieberman et al., 2010; Marzouk \& Najm, 2009). While the application of such techniques to our problem is possible and likely beneficial, most of them require an exploratory offline stage to appraise the true dimensionality of the parameter space. Perhaps a more promising approach is the implementation of trans-dimensional algorithms (e.g. Ray \& Myer, 2019; Brodie \& Jiang, 2018; Bodin \& Sambridge, 2009), which have been shown to be particularly effective in identifying the minimum dimensionality of the model as required by the data itself without the need for an offline appraisal. In particular, the use of Gaussian Processes, within a trans-dimensional context, to generate conductivity models (e.g. Ray \& Myer, 2019) represents a promising approach to also tackle the interpolation cost and spurious oscillations of the Shepard Method. We are currently working on the implementation of these methods.

We would like to point out that, in the case of stand-alone 3D MT inversions, one could combine both the LAB-based and the node-based parameterizations to represent the long-wavelength background and the smaller-scale conductivity anomalies, respectively. In this scenario, the total conductivity distribution will be the addition of the background conductivity obtained with the LAB-based parameterization and the anomalous conductivity distribution represented by nodal values. If the ultimate goal is the total conductivity field, the LAB depths and/or the conductivity nodes can simply be considered nuisance parameters, i.e. they are not of immediate interest but they form part of the problem formulation. The advantage of using the combined parameterization is that rapid convergence is achieved by using LAB depths to constrain the first-order conductivity background, whereas the nodes are used to locally modify this background to fit the smaller-scale features of the data.

For the case of joint inversions of MT with other data sets (e.g. Moorkamp et al., 2007, 2010; Jones et al., 2017; Vozar et al., 2014; Bennington et al., 2015; Jegen et al., 2009; Afonso et al., 2013a,b, 2016a; Jegen et al., 2009; Khan et al., 2008; Afonso et al., 2016b) one would take advantage of the different sensitivities of the inverted observables to the conductivity structure and use complementary paramaterizations. For instance, the background conductivity distribution that results from the longwavelength variation of temperature and bulk composition can be informed by seismic and/or potential field data using their own background parameterization, such as LAB depth, composition and pressure of individual columns of the whole domain (see e.g. Afonso et al., 2013a,b, 2016b; Khan et al., 2008; Jones et al., 2017). The node-based paramaterization could then be used only within the MT forward problem to account for conductivity anomalies superimposed to the background and associated with factors such as fluid content, presence of melt, hydrogen content and anomalous mineral assemblages. A detailed presentation and assessment of this concept are left for a forthcoming publication (Part II). 
M.C. Manassero et al.

\subsection{Efficiency of the RB+MCMC Method}

As mentioned above, a key factor to achieve computational efficiency is to maintain a small number of bases during the MCMC simulation without compromising the quality of their predictions. An alternative to the SVD approach described above is to identify, on-the-fly, the basis vectors that stop contributing to the RB solution (within a predefined threshold) once the MCMC begins to converge to the high probability regions of the parameter space. This information can be obtained by analyzing the vector of coefficients $\mathbf{a}(\theta)$ accompanying the bases (see Eq. 16). Small values for these coefficients indicate minor contributions to the RB solution of the associated basis. Once the uninformative bases (e.g. those created during the burn-in period of the inversion) are identified, they can be eliminated from the reduced basis space. While this would be relatively easy to implement, it requires defining two additional parameters in the $\mathrm{RB}+\mathrm{MCMC}$ algorithm: a minimum threshold value for the coefficients and an interval between successive assessments of the bases (doing it at every MCMC step is extremely inefficient).

Another way of further achieving a small basis size is to seek for accurate solutions only within specific regions of the numerical domain (e.g. Ortega-Gelabert et al., 2020; Alvarez Aramberri, 2015). This stems from the fact that in many practical applications we are not interested in high accuracy at every point inside the numerical box, but rather within a restricted region. A common approach to assess the accuracy of low-fidelity solutions in particular regions of interest is by applying a standard (multi)goal-oriented criterion (Hartmann, 2008; Pardo, 2010; Alvarez Aramberri, 2015; AlvarezAramberri \& Pardo, 2017; Florentin \& Díez, 2012) guided by the error $E_{Q}$ in a diagnostic parameter known as the Quantity of Interest (QoI). The QoI is typically a scalar or vectorial functional of the forward solution that summarizes relevant/important information about the problem at hand. When applied to a restricted region of interest, this goal-oriented criterion results in basis of smaller size relative to those obtained by considering the entire numerical domain (cf. Ortega-Gelabert et al., 2020).

Before ending this section we would like to note that the assembly of the stiffness matrix $\mathbb{K}$ is expensive and including this step at every MCMC iteration is inefficient. When using the LAB-based parameterization, each trial of the MCMC algorithm perturbs the conductivity structure of the model only locally (i.e. within a column). In this case, only a small number of elements of the matrix $\mathbb{K}$ varies between proposed samples, which in turn contribute to a small and local change of the matrix $\mathbb{K}_{\mathbb{R B B}}$. We have taken advantage of this and implemented an algorithm that directly updates the corresponding elements in $\mathbb{K}_{\mathbb{R} \mathbb{B}}$ (for every frequency) instead of re-computing the full $\mathbb{K}$ for every trial. This strategy is also possible within the node-based parameterization. However, in the present implementation, the Shepard interpolation does not provide a mapping between a node that changes value and the position of the perturbed cells in the conductivity model. This limitation can be overcome using other interpo- 
lation strategies where this mapping is available, as in the case of Gaussian processes (Ray \& Myer, 2019).

\section{CONCLUSIONS}

We have developed a novel approach for MCMC-driven probabilistic inversions of 3D magnetotelluric (MT) data. The success of the method relies on the combination of a reduced order technique (Reduced Basis Method) to create fast and accurate surrogates, an efficient parallelization of the forward problem and adaptive strategies for both the surrogate and the MCMC algorithm. Unlike traditional implementations, the surrogate construction and adaptation (refinement) process is integrated into the MCMC inversion, thus removing the need for costly offline stages.

The feasibility and performance of the proposed approach to invert for large-scale conductivity structures are demonstrated with two inversion examples with different parameterizations and dimensionalities. In both cases, the RB+MCMC approach successfully solves the probabilistic inverse problem using less than $\sim 5 \%$ of the computational cost needed in a conventional MCMC approach. Furthermore, the efficiency of the method increases with the length of the MCMC simulation, as fewer basis enrichments are necessary once the chain begins to converge.

We note that the type of model parameterization and chosen MCMC scheme can significantly impact both the posterior PDFs and the convergence of the MCMC chain. In this work, we purposely adopted the simplest parameterizations and MCMC schemes. The efficiency of our strategy can therefore be further improved by making use of more advanced parameterization techniques and MCMC algorithms (e.g. tras-dimensional schemes, Gaussian Processes, delayed rejection, parallel tempering, differential evolution, among others).

As a by-product, we have obtained an optimized version (Max3D-G) of the original MT finite element forward code developed by Zyserman \& Santos (2000). The new implementation solves the finite element linear system of equations using the parallel solver MUMPS which reduces the computational time by $\sim 80 \%$ compared to the original code.

Our new RB+MCMC approach makes 3D MT probabilistic inversions a practical option and opens up new exciting opportunities for both stand-alone MT studies and integrated geophysical imaging of the Earth's interior via joint inversions (e.g Afonso et al., 2013b,a; Khan et al., 2008; Afonso et al., 2016a). We will explore the latter possibility in a forthcoming publication (Part II). 


\section{M.C. Manassero et al.}

\section{SUPPLEMENTARY MATERIAL}

The online supplementary material contains benchmarks of the optimized version (Max3D-G) of the original MT finite element forward code developed by Zyserman \& Santos (2000) and an application of our $\mathrm{RB}+\mathrm{MCMC}$ approach to a heat transfer problem. We also present additional results of the numerical examples showed in Sections 6.2 and 6.3.

\section{ACKNOWLEDGMENTS}

We thank Farshad Salajegheh for providing part of his Matlab codes for plotting results and Sinan Özaydin for running the ModEM inversions. Special thanks to Olga Ortega-Gelabert, Kate Selway, Stephan Thiel, Alan Jones, Anandaroop Ray, Marina Rosas-Carbajal, Juanjo Ledo, Javier Fullea and Beñat Oliveira for their comments and contributions at different stages of this work. We thank Naser Meqbel for providing the 3D Grid tool used to create the models shown in Supplementary Material. The 3D rendering views were created using ParaView (Ahrens et al., 2005).

MCM thanks support from an International Macquarie Research Excellence Scholarship (iMQRES). MCM and JCA acknowledge support from ARC Grant DP160103502, ARC Linkage Grant LP170100233, the ARC Centre of Excellence Core to Crust Fluids Systems (http://www.ccfs.mq.edu.au) and the Centre for Earth Evolution and Dynamics, Geoscience Australia and the European Space Agency via the “3D Earth - A Dynamic Living Planet”. FZ acknowledges support from CONICET through grant PIP 112-201501-00192. SZ has been funded by the Spanish Ministry through grant DPI2017-85139-C22-R, by Catalan government through grant 2017-SGR-1278 and by the EU's Horizon 2020 research and innovation programme under the Marie Sklodowska-Curie grant agreement No 777778.

\section{References}

Afonso, J., Fullea, J., Griffin, W., Yang, Y., Jones, A., Connolly, J., \& O’Reilly, S., 2013a. 3-D multiobservable probabilistic inversion for the compositional and thermal structure of the lithosphere and upper mantle. I: A priori petrological information and geophysical observables, Journal of Geophysical Research: Solid Earth, 118(5), 2586-2617.

Afonso, J. C., Fullea, J., Yang, Y., Connolly, J., \& Jones, A., 2013b. 3-D multi-observable probabilistic inversion for the compositional and thermal structure of the lithosphere and upper mantle. II: General methodology and resolution analysis, Journal of Geophysical Research: Solid Earth, 118(4), 1650-1676.

Afonso, J. C., Moorkamp, M., \& Fullea, J., 2016a. Imaging the lithosphere and upper mantle: Where 
we are at and where we are going, Integrated Imaging of the Earth: Theory and Applications, 218, 191.

Afonso, J. C., Rawlinson, N., Yang, Y., Schutt, D. L., Jones, A. G., Fullea, J., \& Griffin, W. L., 2016b. 3-D multiobservable probabilistic inversion for the compositional and thermal structure of the lithosphere and upper mantle: III. Thermochemical tomography in the Western-Central US, Journal of Geophysical Research: Solid Earth, 121(10), 7337-7370.

Ahrens, J., Geveci, B., \& Law, C., 2005. Paraview: An end-user tool for large data visualization, The visualization handbook, 717.

Alvarez Aramberri, J., 2015. hp-Adaptive simulation and inversion of magnetotelluric measurements, Ph.D. thesis, Pau.

Alvarez-Aramberri, J. \& Pardo, D., 2017. Dimensionally adaptive hp-finite element simulation and inversion of 2D magnetotelluric measurements, Journal of Computational Science, 18, 95-105.

Amestoy, P. R., Duff, I. S., L'Excellent, J.-Y., \& Koster, J., 2001. A fully asynchronous multifrontal solver using distributed dynamic scheduling, SIAM Journal on Matrix Analysis and Applications, 23(1), 15-41.

Amestoy, P. R., Guermouche, A., L'Excellent, J.-Y., \& Pralet, S., 2006. Hybrid scheduling for the parallel solution of linear systems, Parallel computing, 32(2), 136-156.

Aster, R. C., Borchers, B., \& Thurber, C. H., 2018. Parameter estimation and inverse problems, Elsevier.

Avdeev, D. \& Avdeeva, A., 2009. 3D magnetotelluric inversion using a limited-memory quasiNewton optimization, Geophysics, 74(3), F45-F57.

Avdeev, D. B., 2005. Three-dimensional electromagnetic modelling and inversion from theory to application, Surveys in Geophysics, 26(6), 767-799.

Benner, P., Ohlberger, M., Patera, A., Rozza, G., \& Urban, K., 2017. Model Reduction of Parametrized Systems, Springer.

Bennington, N. L., Zhang, H., Thurber, C. H., \& Bedrosian, P. A., 2015. Joint inversion of seismic and magnetotelluric data in the Parkfield Region of California using the normalized cross-gradient constraint, Pure and Applied Geophysics, 172(5), 1033-1052.

Berdichevsky, M. N. \& Dmitriev, V. I., 2010. Models and methods of magnetotellurics, Springer Science \& Business Media.

Bodin, T. \& Sambridge, M., 2009. Seismic tomography with the reversible jump algorithm, Geophysical Journal International, 178(3), 1411-1436.

Brodie, R. \& Jiang, W., 2018. Trans-dimensional Monte Carlo inversion of short period magnetotelluric data for cover thickness estimation, ASEG Extended Abstracts, 2018(1), 1-7. 
Brunton, S. L. \& Kutz, J. N., 2019. Data-driven science and engineering: Machine learning, dynamical systems, and control, Cambridge University Press.

Cagniard, L., 1953. Basic theory of the magneto-telluric method of geophysical prospecting, Geophysics, 18(3), 605-635.

Chen, J., Hoversten, G. M., Key, K., Nordquist, G., \& Cumming, W., 2012. Stochastic inversion of magnetotelluric data using a sharp boundary parameterization and application to a geothermal site, Geophysics, 77(4), E265-E279.

Chen, Y., Hesthaven, J. S., Maday, Y., \& Rodríguez, J., 2010. Certified reduced basis methods and output bounds for the harmonic Maxwell's Equations, SIAM Journal on Scientific Computing, 32(2), 970-996.

Comeau, M. J., Unsworth, M. J., \& Cordell, D., 2016. New constraints on the magma distribution and composition beneath Volcán Uturuncu and the southern Bolivian Altiplano from magnetotelluric data, Geosphere, 12(5), 1391-1421.

Conway, D., Simpson, J., Didana, Y., Rugari, J., \& Heinson, G., 2018. Probabilistic Magnetotelluric Inversion with Adaptive Regularisation Using the No-U-Turns Sampler, Pure and Applied Geophysics, pp. 1-14.

Cui, T., Marzouk, Y. M., \& Willcox, K. E., 2015. Data-driven model reduction for the Bayesian solution of inverse problems, International Journal for Numerical Methods in Engineering, 102(5), 966-990.

Dai, L. \& Karato, S.-i., 2009. Electrical conductivity of orthopyroxene: Implications for the water content of the asthenosphere, Proceedings of the Japan Academy, Series B, 85(10), 466-475.

deGroot Hedlin, C. \& Constable, S., 1990. Occam's inversion to generate smooth, two-dimensional models from magnetotelluric data, Geophysics, 55(12), 1613-1624.

Douglas Jr, J., Santos, J. E., Sheen, D., \& Ye, X., 1999. Nonconforming galerkin methods based on quadrilateral elements for second order elliptic problems, ESAIM: Mathematical Modelling and Numerical Analysis, 33(4), 747-770.

Douglas Jr, J., Santos, J. E., \& Sheen, D., 2000. A nonconforming mixed finite element method for Maxwell's Equations, Mathematical Models and Methods in Applied Sciences, 10(04), 593-613.

Egbert, G. D. \& Kelbert, A., 2012. Computational recipes for electromagnetic inverse problems, Geophysical Journal International, 189(1), 251-267.

Evans, R., 2012. Conductivity of Earth materials, The Magnetotelluric Method, Theory and Practice, pp. 50-95.

Evans, R. L., Hirth, G., Baba, K., Forsyth, D., Chave, A., \& Mackie, R., 2005. Geophysical evidence from the MELT area for compositional controls on oceanic plates, Nature, 437(7056), 249. 
Farquharson, C. G., Oldenburg, D. W., Haber, E., \& Shekhtman, R., 2002. An algorithm for the three-dimensional inversion of magnetotelluric data, in SEG Technical Program Expanded Abstracts 2002, pp. 649-652, Society of Exploration Geophysicists.

Florentin, E. \& Díez, P., 2012. Adaptive reduced basis strategy based on goal oriented error assessment for stochastic problems, Computer Methods in Applied Mechanics and Engineering, 225, $116-127$.

Frangos, M., Marzouk, Y., Willcox, K., \& van Bloemen Waanders, B., 2010. Surrogate and reducedorder modeling: a comparison of approaches for large-scale statistical inverse problems [chapter 7].

Fullea, J., Muller, M., \& Jones, A., 2011. Electrical conductivity of continental lithospheric mantle from integrated geophysical and petrological modeling: Application to the Kaapvaal Craton and Rehoboth Terrane, southern Africa, Journal of Geophysical Research: Solid Earth, 116(B10).

Gilks, W. R., Richardson, S., \& Spiegelhalter, D., 1995. Markov chain Monte Carlo in practice, Chapman and Hall/CRC.

Gregory, P., 2005. Bayesian Logical Data Analysis for the Physical Sciences: A Comparative Ap-

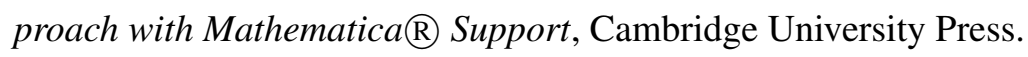

Haario, H., Saksman, E., Tamminen, J., et al., 2001. An adaptive Metropolis algorithm, Bernoulli, 7(2), 223-242.

Haario, H., Laine, M., Mira, A., \& Saksman, E., 2006. DRAM: efficient adaptive MCMC, Statistics and computing, 16(4), 339-354.

Haber, E., Ascher, U. M., \& Oldenburg, D. W., 2004. Inversion of 3D electromagnetic data in frequency and time domain using an inexact all-at-once approach, Geophysics, 69(5), 1216-1228.

Han, N., Nam, M. J., Kim, H. J., Lee, T. J., Song, Y., \& Suh, J. H., 2008. Efficient three-dimensional inversion of magnetotelluric data using approximate sensitivities, Geophysical Journal International, 175(2), 477-485.

Harinarayana, T., 1999. Combination of em and dc measurements for upper crustal studies, Surveys in Geophysics, 20(3-4), 257-278.

Hartmann, R., 2008. Multitarget error estimation and adaptivity in aerodynamic flow simulations, SIAM Journal on Scientific Computing, 31(1), 708-731.

Hashin, Z. \& Shtrikman, S., 1962. A variational approach to the theory of the effective magnetic permeability of multiphase materials, Journal of applied Physics, 33(10), 3125-3131.

Hashin, Z. \& Shtrikman, S., 1963. A variational approach to the theory of the elastic behaviour of multiphase materials, Journal of the Mechanics and Physics of Solids, 11(2), 127-140.

Hastings, W. K., 1970. Monte Carlo sampling methods using Markov chains and their applications. 
Heise, W., Caldwell, T., Bibby, H. M., \& Bannister, S., 2008. Three-dimensional modelling of magnetotelluric data from the rotokawa geothermal field, Taupo Volcanic Zone, New Zealand, Geophysical Journal International, 173(2), 740-750.

Hess, M. W., 2016. Reduced Basis Approximations for Electromagnetic Applications, Ph.D. thesis, Otto-von-Guericke Universität Magdeburg.

Hess, M. W. \& Benner, P., 2013. Fast evaluation of time-harmonic Maxwell's Equations using the reduced basis method, IEEE Transactions on Microwave Theory and Techniques, 61(6), 2265-2274.

Hesthaven, J. S., Stamm, B., \& Zhang, S., 2012. Certified reduced basis method for the electric field integral equation, SIAM Journal on Scientific Computing, 34(3), A1777-A1799.

Hesthaven, J. S., Rozza, G., Stamm, B., et al., 2016. Certified reduced basis methods for parametrized partial differential Equations, Springer.

Hoffmann, R. \& Dietrich, P., 2004. An approach to determine equivalent solutions to the geoelectrical 2d inversion problem, Journal of Applied Geophysics, 56(2), 79-91.

Jegen, M. D., Hobbs, R. W., Tarits, P., \& Chave, A., 2009. Joint inversion of marine magnetotelluric and gravity data incorporating seismic constraints: Preliminary results of sub-basalt imaging off the Faroe Shelf, Earth and Planetary Science Letters, 282(1-4), 47-55.

Jones, A. G., Afonso, J. C., \& Fullea, J., 2017. Geochemical and geophysical constrains on the dynamic topography of the Southern African Plateau, Geochemistry, Geophysics, Geosystems, 18(10), 3556-3575.

Karato, S.-I., 1990. The role of hydrogen in the electrical conductivity of the upper mantle, Nature, 347(6290), 272.

Karato, S.-i., 2011. Water distribution across the mantle transition zone and its implications for global material circulation, Earth and Planetary Science Letters, 301(3-4), 413-423.

Karato, S.-i. \& Wang, D., 2013. Electrical conductivity of minerals and rocks, Physics and Chemistry of the Deep Earth, pp. 145-182.

Kelbert, A., Egbert, G. D., \& Schultz, A., 2008. Non-linear conjugate gradient inversion for global EM induction: resolution studies, Geophysical Journal International, 173(2), 365-381.

Kelbert, A., Schultz, A., \& Egbert, G., 2009. Global electromagnetic induction constraints on transition-zone water content variations, Nature, 460(7258), 1003-1006.

Kelbert, A., Meqbel, N., Egbert, G. D., \& Tandon, K., 2014. ModEM: A modular system for inversion of electromagnetic geophysical data, Computers \& Geosciences, 66, 40-53.

Key, K., 2016. MARE2DEM: a 2-D inversion code for controlled-source electromagnetic and magnetotelluric data, Geophysical Journal International, 207(1), 571-588.

Khan, A., 2016. On Earth's mantle constitution and structure from joint analysis of geophysical and 
laboratory-based data: An example, Surveys in Geophysics, 37(1), 149-189.

Khan, A., Connolly, J., \& Taylor, S., 2008. Inversion of seismic and geodetic data for the major element chemistry and temperature of the Earth's mantle, Journal of Geophysical Research: Solid Earth, 113(B9).

Kuvshinov, A., 2008. 3-D global induction in the oceans and solid Earth: recent progress in modeling magnetic and electric fields from sources of magnetospheric, ionospheric and oceanic origin, Surveys in Geophysics, 29(2), 139-186.

Kuvshinov, A. \& Olsen, N., 2006. A global model of mantle conductivity derived from 5 years of CHAMP, Ørsted, and SAC-C magnetic data, Geophysical Research Letters, 33(18).

Kuvshinov, A. \& Semenov, A., 2012. Global 3-D imaging of mantle electrical conductivity based on inversion of observatory C-responses-I. an approach and its verification, Geophysical Journal International, 189(3), 1335-1352.

Kuvshinov, A., Sabaka, T., \& Olsen, N., 2006. 3-D electromagnetic induction studies using the Swarm constellation: Mapping conductivity anomalies in the Earth's mantle, Earth, planets and space, 58(4), 417-427.

Lieberman, C., Willcox, K., \& Ghattas, O., 2010. Parameter and state model reduction for large-scale statistical inverse problems, SIAM Journal on Scientific Computing, 32(5), 2523-2542.

Mackie, R. L. \& Madden, T. R., 1993. Three-dimensional magnetotelluric inversion using conjugate gradients, Geophysical Journal International, 115(1), 215-229.

Mackie, R. L., Madden, T. R., \& Park, S. K., 1996. A three-dimensional magnetotelluric investigation of the California Basin and Range, Journal of Geophysical Research: Solid Earth, 101(B7), 1622116239.

Mallick, K. \& Verma, R., 1979. Time-domain electromagnetic sounding-computation of multilayer response and the problem of equivalence in interpretation, Geophysical Prospecting, 27(1), 137-155.

Marzouk, Y. M. \& Najm, H. N., 2009. Dimensionality reduction and polynomial chaos acceleration of Bayesian inference in inverse problems, Journal of Computational Physics, 228(6), 1862-1902.

Meqbel, N. M., Egbert, G. D., Wannamaker, P. E., Kelbert, A., \& Schultz, A., 2014. Deep electrical resistivity structure of the northwestern us derived from 3-D inversion of USArray magnetotelluric data, Earth and Planetary Science Letters, 402, 290-304.

Metropolis, N., Rosenbluth, A. W., Rosenbluth, M. N., Teller, A. H., \& Teller, E., 1953. Equation of state calculations by fast computing machines, The journal of chemical physics, 21(6), 1087-1092. Mira, A. et al., 2001. On Metropolis-Hastings algorithms with delayed rejection, Metron, 59(3-4), 231-241. 
Moorkamp, M., Jones, A., \& Eaton, D., 2007. Joint inversion of teleseismic receiver functions and magnetotelluric data using a genetic algorithm: Are seismic velocities and electrical conductivities compatible?, Geophysical Research Letters, 34(16).

Moorkamp, M., Jones, A., \& Fishwick, S., 2010. Joint inversion of receiver functions, surface wave dispersion, and magnetotelluric data, Journal of Geophysical Research: Solid Earth, 115(B4).

Mosegaard, K. \& Hansen, T. M., 2016. Inverse methods: Problem formulation and probabilistic solutions, Integrated Imaging of the Earth: Theory and Applications, Geophysical Monograph, 218, 9-27.

Newman, G. A. \& Alumbaugh, D. L., 2000. Three-dimensional magnetotelluric inversion using non-linear conjugate gradients, Geophysical journal international, 140(2), 410-424.

Newman, G. A. \& Boggs, P. T., 2004. Solution accelerators for large-scale three-dimensional electromagnetic inverse problems, Inverse Problems, 20(6), S151.

Ogawa, Y., Mishina, M., Goto, T., Satoh, H., Oshiman, N., Kasaya, T., Takahashi, Y., Nishitani, T., Sakanaka, S., Uyeshima, M., et al., 2001. Magnetotelluric imaging of fluids in intraplate earthquake zones, NE Japan back arc, Geophysical research letters, 28(19), 3741-3744.

Olsen, N., Lühr, H., Sabaka, T. J., Mandea, M., Rother, M., Tøffner-Clausen, L., \& Choi, S., 2006. CHAOS—a model of the Earth's magnetic field derived from CHAMP, Ørsted, and SAC-C magnetic satellite data, Geophysical Journal International, 166(1), 67-75.

Olsen, N., Friis-Christensen, E., Floberghagen, R., Alken, P., Beggan, C. D., Chulliat, A., Doornbos, E., Da Encarnação, J. T., Hamilton, B., Hulot, G., et al., 2013. The Swarm satellite constellation application and research facility (SCARF) and swarm data products, Earth, Planets and Space, $\mathbf{6 5}(11), 1$.

Ortega-Gelabert, O., Zlotnik, S., Afonso, J., \& Déez, P., 2020. Fast Stokes flow simulations for geophysical-geodynamic inverse problems and sensitivity analyses based on reduced order modeling, Journal of Geophysical Research: Solid Earth, p. e2019JB018314.

Pardo, D., 2010. Multigoal-oriented adaptivity for hp-finite element methods, Procedia Computer Science, 1(1), 1953-1961.

Park, S. K. \& Ostos, L. C., 2013. Constraints from magnetotelluric measurements on magmatic processes and upper mantle structure in the vicinity of lassen volcanic center, northern california, Geosphere, 9(3), 382-393.

Patera, A. T., Rozza, G., et al., 2007. Reduced basis approximation and a posteriori error estimation for parametrized partial differential equations.

Peherstorfer, B., Willcox, K., \& Gunzburger, M., 2018. Survey of multifidelity methods in uncertainty propagation, inference, and optimization, SIAM Review, 60(3), 550-591. 
Pommier, A., 2014. Interpretation of magnetotelluric results using laboratory measurements, Surveys in Geophysics, 35(1), 41-84.

Prud'Homme, C., Rovas, D. V., Veroy, K., Machiels, L., Maday, Y., Patera, A. T., \& Turinici, G., 2002. Reliable real-time solution of parametrized partial differential Equations: Reduced-basis output bound methods, Journal of Fluids Engineering, 124(1), 70-80.

Quarteroni, A., Rozza, G., \& Manzoni, A., 2011. Certified reduced basis approximation for parametrized partial differential Equations and applications, Journal of Mathematics in Industry, 1(1), 3 .

Quarteroni, A., Manzoni, A., \& Negri, F., 2015. Reduced basis methods for partial differential Equations: an introduction, vol. 92, Springer.

Ray, A. \& Myer, D., 2019. Bayesian geophysical inversion with trans-dimensional Gaussian process machine learning, Geophysical Journal International, 217(3), 1706-1726.

Renka, R. J., 1988a. Algorithm 661: QSHEP3D: Quadratic Shepard method for trivariate interpolation of scattered data, ACM Transactions on Mathematical Software (TOMS), 14(2), 151-152.

Renka, R. J., 1988b. Multivariate interpolation of large sets of scattered data, ACM Transactions on Mathematical Software (TOMS), 14(2), 139-148.

Roberts, J. J. \& Tyburczy, J. A., 1999. Partial-melt electrical conductivity: Influence of melt composition, Journal of Geophysical Research: Solid Earth, 104(B4), 7055-7065.

Robertson, K., Thiel, S., \& Meqbel, N., 2020. Quality over quantity: on workflow and model space exploration of 3d inversion of mt data, Earth, Planets and Space, 72(1), 1-22.

Rosas-Carbajal, M., Linde, N., Kalscheuer, T., \& Vrugt, J. A., 2013. Two-dimensional probabilistic inversion of plane-wave electromagnetic data: methodology, model constraints and joint inversion with electrical resistivity data, Geophysical Journal International, 196(3), 1508-1524.

Rosas-Carbajal, M., Linde, N., Peacock, J., Zyserman, F. I., Kalscheuer, T., \& Thiel, S., 2015. Probabilistic 3-D time-lapse inversion of magnetotelluric data: application to an enhanced geothermal system, Geophysical Supplements to the Monthly Notices of the Royal Astronomical Society, 203(3), 1946-1960.

Rozza, G., Huynh, D. B. P., \& Patera, A. T., 2007. Reduced basis approximation and a posteriori error estimation for affinely parametrized elliptic coercive partial differential Equations, Archives of Computational Methods in Engineering, 15(3), 1.

Rozza, G., Huynh, D., Nguyen, N. C., \& Patera, A. T., 2009. Real-time reliable simulation of heat transfer phenomena, in Proceedings of the ASME HT 2009 summer conference, no. CONF, ASME. Rozza, G., Huynh, D. P., \& Manzoni, A., 2013. Reduced basis approximation and a posteriori error estimation for stokes flows in parametrized geometries: roles of the inf-sup stability constants, 
M.C. Manassero et al.

Numerische Mathematik, 125(1), 115-152.

Sabaka, T. J., Olsen, N., \& Purucker, M. E., 2004. Extending comprehensive models of the Earth's magnetic field with Ørsted and CHAMP data, Geophysical Journal International, 159(2), 521-547.

Sasaki, Y., 2001. Full 3-D inversion of electromagnetic data on pc, Journal of Applied Geophysics, 46(1), 45-54.

Selway, K., 2014. On the causes of electrical conductivity anomalies in tectonically stable lithosphere, Surveys in Geophysics, 35(1), 219-257.

Selway, K., O’Donnell, J., \& Özaydin, S., 2019. Upper mantle melt distribution from petrologically constrained magnetotellurics, Geochemistry, Geophysics, Geosystems, 20(7), 3328-3346.

Semenov, A. \& Kuvshinov, A., 2012. Global 3-D imaging of mantle conductivity based on inversion of observatory C-responses-II. data analysis and results, Geophysical Journal International, 191(3), 965-992.

Sen, M. K. \& Stoffa, P. L., 1996. Bayesian inference, Gibbs' sampler and uncertainty estimation in geophysical inversion, Geophysical Prospecting, 44(2), 313-350.

Sheen, D., 1997. Approximation of electromagnetic fields: Part I. Continuous problems, SIAM Journal on Applied Mathematics, 57(6), 1716-1736.

Shen, Y., Xu, P., \& Li, B., 2012. Bias-corrected regularized solution to inverse ill-posed models, Journal of Geodesy, 86(8), 597-608.

Siripunvaraporn, W., 2012. Three-dimensional magnetotelluric inversion: an introductory guide for developers and users, Surveys in geophysics, 33(1), 5-27.

Siripunvaraporn, W. \& Egbert, G., 2009. WSINV3DMT: vertical magnetic field transfer function inversion and parallel implementation, Physics of the Earth and Planetary Interiors, 173(3-4), 317329.

Siripunvaraporn, W., Egbert, G., Lenbury, Y., \& Uyeshima, M., 2005. Three-dimensional magnetotelluric inversion: data-space method, Physics of the Earth and planetary interiors, 150(1-3), 3-14.

Tarantola, A., 2005. Inverse problem theory and methods for model parameter estimation, vol. 89, siam.

Tarantola, A., Valette, B., et al., 1982. Inverse problems= quest for information, Journal of geophysics, 50(1), 159-170.

Ten Grotenhuis, S. M., Drury, M. R., Spiers, C. J., \& Peach, C. J., 2005. Melt distribution in olivine rocks based on electrical conductivity measurements, Journal of Geophysical Research: Solid Earth, 110(B12).

Tietze, K. \& Ritter, O., 2013. Three-dimensional magnetotelluric inversion in practice-the electrical conductivity structure of the San Andreas Fault in Central California, Geophysical Journal 
International, 195(1), 130-147.

Tikhonov, A., 1950. On determining electric characteristics of the deep layers of the earth's crust, Dolk. Acad. Nauk. SSSR, 73, 295-297.

Varentsov, I. M., Kulikov, V., Yakovlev, A., \& Yakovlev, D., 2013. Possibilities of magnetotelluric methods in geophysical exploration for ore minerals, Izvestiya, Physics of the Solid Earth, 49(3), 309-328.

Vozar, J., Jones, A. G., Fullea, J., Agius, M. R., Lebedev, S., Le Pape, F., \& Wei, W., 2014. Integrated geophysical-petrological modeling of lithosphere-asthenosphere boundary in central Tibet using electromagnetic and seismic data, Geochemistry, Geophysics, Geosystems, 15(10), 3965-3988.

Vozoff, K., 1990. Magnetotellurics: Principles and practice, Proceedings of the Indian Academy of Sciences-Earth and Planetary Sciences, 99(4), 441-471.

Wait, J. R., 1962. Theory of magnetotelluric fields, J. Res. NBS D, 66(5), 509-541.

Wang, D., Mookherjee, M., Xu, Y., \& Karato, S.-i., 2006. The effect of water on the electrical conductivity of olivine, Nature, $\mathbf{4 4 3 ( 7 1 1 4 ) , 9 7 7 .}$

Wei, W., Unsworth, M., Jones, A., Booker, J., Tan, H., Nelson, D., Chen, L., Li, S., Solon, K., Bedrosian, P., et al., 2001. Detection of widespread fluids in the Tibetan crust by magnetotelluric studies, Science, 292(5517), 716-719.

Yan, L. \& Zhou, T., 2019. Adaptive multi-fidelity polynomial chaos approach to bayesian inference in inverse problems, Journal of Computational Physics, 381, 110-128.

Yang, B., Egbert, G. D., Kelbert, A., \& Meqbel, N. M., 2015. Three-dimensional electrical resistivity of the north-central USA from Earthscope long period magnetotelluric data, Earth and Planetary Science Letters, 422, 87-93.

Yoshino, T., 2010. Laboratory electrical conductivity measurement of mantle minerals, Surveys in Geophysics, 31(2), 163-206.

Yoshino, T., Matsuzaki, T., Shatskiy, A., \& Katsura, T., 2009. The effect of water on the electrical conductivity of olivine aggregates and its implications for the electrical structure of the upper mantle, Earth and Planetary Science Letters, 288(1-2), 291-300.

Yoshino, T., Shimojuku, A., Shan, S., Guo, X., Yamazaki, D., Ito, E., Higo, Y., \& Funakoshi, K.-i., 2012. Effect of temperature, pressure and iron content on the electrical conductivity of olivine and its high-pressure polymorphs, Journal of Geophysical Research: Solid Earth, 117(B8).

Zhang, J., Zheng, Q., Chen, D., Wu, L., \& Zeng, L., 2019. Surrogate-based bayesian inverse modeling of the hydrological system: An adaptive approach considering surrogate approximation error, Water Resources Research.

Zhdanov, M. \& Hursan, G., 2000. 3D electromagnetic inversion based on quasi-analytical approxi- 


\section{M.C. Manassero et al.}

mation, Inverse Problems, 16(5), 1297.

Zhdanov, M. S. \& Keller, G. V., 1994. The geoelectrical methods in geophysical exploration, Methods in geochemistry and geophysics, 31, I-IX.

Zhdanov, M. S., Fang, S., \& Hursán, G., 2000. Electromagnetic inversion using quasi-linear approximation, Geophysics, 65(5), 1501-1513.

Zhdanov, M. S., Green, A., Gribenko, A., \& Cuma, M., 2010. Large-scale three-dimensional inversion of Earthscope MT data using the integral equation method, Izvestiya, Physics of the solid Earth, 46(8), 670-678.

Zyserman, F. I. \& Santos, J. E., 2000. Parallel finite element algorithm with domain decomposition for three-dimensional magnetotelluric modelling, Journal of Applied Geophysics, 44(4), 337-351.

\section{APPENDIX A: MAPPING THERMOCHEMICAL PARAMETERS TO ELECTRICAL CONDUCTIVITY}

The electrical conductivity of mantle rocks is primarily controlled by their thermo-physical state (temperature, pressure, composition, fluid, iron and melt content). The temperature dependence of electrical conductivity can be described with an Arrhenius-type Equation:

$$
\sigma=\sigma_{0} \exp \left(\frac{-\Delta H}{k_{B} T}\right)
$$

where $\sigma_{0}$ is the so-called pre-exponential factor, $T[K]$ is absolute temperature and $k_{B}[\mathrm{eV} / \mathrm{K}]$ the Boltzmann's constant. $\Delta H[e V]$ is the activation enthalpy, which can be defined as a function of pressure $P[G P a]$ in the following way:

$$
\Delta H=\Delta E+P \Delta V
$$

where $\Delta E$ and $\Delta V$ are the activation energy and activation volume, respectively.

The main bulk conduction mechanisms in mantle minerals are ionic conduction, small polaron (hopping) conduction and proton conduction. Each mechanism follows an Arrhenius-type equation with particular activation energies depending on their charge mobility. These three conduction mechanisms can be integrated in a model for the electrical conductivity of mantle rocks as a function of pressure, temperature, water content, and composition (via Fe content) for each mineral phase (see also Yoshino et al., 2009; Fullea et al., 2011): 


\begin{tabular}{lccccccccccc}
\hline Phase & $\sigma_{0}$ & $\sigma_{0 i}$ & $\mathrm{a}$ & $\mathrm{b}$ & $\mathrm{c}$ & $\mathrm{d}$ & $\mathrm{e}$ & $\mathrm{f}$ & $\Delta V$ & $\Delta H_{i}$ & $X_{F e}$ \\
\hline Olivine & 2.70 & 4.73 & 1.64 & 0.246 & -4.85 & 3.26 & & & 0.68 & 2.31 & 0.10 \\
Opx & 3.0 & & 1.90 & -2.77 & 2.61 & -1.09 & & & & & 0.107 \\
Cpx & 3.25 & & 2.07 & -2.77 & 2.61 & -1.09 & & & & & $5.84 e^{-2}$ \\
Garnet & & 4.96 & 2.60 & -15.33 & 80.40 & -194.6 & 202.6 & -75.0 & & & 0.168 \\
\hline
\end{tabular}

Table A1. Parameters used to compute the conductivity of the mantle

$$
\begin{aligned}
\sigma & =\sigma_{0} \exp \left(\frac{-\Delta H\left(X_{F e}, P\right)}{k_{B} T}\right)+\sigma_{0 i} \exp \left(\frac{-\Delta H_{i}}{k_{B} T}\right)+\sigma_{p} \\
\sigma_{p} & =f\left(C_{w}\right) \exp \left(\frac{-\Delta H_{w e t}\left(C_{w}\right)}{k_{B} T}\right) \\
-\Delta H\left(X_{F e}, P\right) & =a+b X_{F e}+c X_{F e}^{2}+d X_{F e}^{3}+e X_{F e}^{4}+f X_{F e}^{5}+P \Delta V,
\end{aligned}
$$

where $\sigma_{0}, \sigma_{0 i}[S / m]$ and $f\left(C_{w}\right)$ are the so-called small polaron, ionic and proton pre-exponential factors, respectively; $\Delta V\left[\mathrm{~cm}^{3} / \mathrm{mol}\right]$ is activation volume; $\Delta H, \Delta H_{i}[\mathrm{eV}]$ and $\Delta H_{\text {wet }}$ are activation enthalpies; $X_{F e}$ is the bulk Fe content in wt\%; $T[K]$ is absolute temperature; $P[G P a]$ is pressure and $k_{B}[e V / K]$ the Boltzmann's constant.

The first term of the right-hand side of Equation A.3a describes the contribution from small polaron conduction. As mentioned above, the activation enthalpy for this process depends on the iron content and pressure. This dependence is represented by a polynomial on $X_{F e}$ (Eq. A.3c) plus a term that depends on pressure (the coefficients $a, b, c, d, e, f$ are determined experimentally). The second term of Equation A.3a represents ionic conduction at high temperature and the third term $\left(\sigma_{p}\right)$ represents the proton conduction due to the presence of "water" (hydrogen diffusion). $f\left(C_{w}\right)$ and $\Delta H_{w e t}$ are functions of the water content $C_{w}[w t \%]$ and they are estimated from laboratory experiments. The reader is referred to Fullea et al. (2011) and Pommier (2014) for a summary of results from different laboratories.

\section{APPENDIX B: PROPOSAL AND PRIOR DISTRIBUTIONS}

In the particular case of uncorrelated parameters, the proposal probability distribution function (PDF) for a sample $\mathbf{m}_{t}=\left[m_{t}^{1}, . ., m_{t}^{N}\right]$ is

$$
q\left(\mathbf{m}_{t} \mid \mathbf{m}_{t-1}\right)=\prod_{i=1}^{N} q\left(m_{t}^{i} \mid m_{t-1}^{i}\right),
$$


M.C. Manassero et al.

where $N$ is the number of parameters and $q\left(m^{i} \mid m_{t-1}^{i}\right)$ is the probability of the proposed move $m_{t}^{i}$ for the $i$-th parameter conditional on its current state $m_{t-1}^{i}$. In the particular case of a symmetric proposal, such as Gaussian or uniform PDFs, it satisfies $q\left(m_{t}^{i} \mid m_{t-1}^{i}\right)=q\left(m_{t-1}^{i} \mid m_{t}^{i}\right)$ for all $m_{t}^{i}$ and $m_{t-1}^{i}$.

In the same way, assuming uncorrelated parameters as prior information, the prior probability of a sample $\mathbf{m}_{t}$ is

$$
P\left(\mathbf{m}_{t}\right)=\prod_{i=1}^{N} P\left(m_{t}^{i}\right),
$$

where $P\left(m_{t}^{i}\right)$ corresponds to the prior for the $i$-th parameter. In the case of uniform prior PDFs we have that $P\left(m_{t}^{i}\right)=1$, and in the case of a Gaussian PDF:

$$
P\left(m_{t}^{i}\right)=\frac{1}{s \sqrt{2 \pi}} \exp \left(-\frac{\left(m_{t}^{i}-\mu\right)^{2}}{2 s^{2}}\right),
$$

where $\mu$ and $s$ are the mean and standard deviation,respectively.

In situations where the values of the model parameters span several orders of magnitude, it is useful to define prior and proposal distributions in logarithmic scale such as the Jeffreys' PDF (uniform in log-scale ) with associated probability $y\left(m_{t}^{i}\right)=1 / m_{t}^{i}$ (Gregory, 2005). Another common choice is to use log-normal distributions (Gaussian in log-scale):

$$
y\left(m_{t}^{i}\right)=\frac{1}{\sqrt{2 \pi} m_{t}^{i} s} \exp \left(-\frac{\ln \left(m_{t}^{i}\right)-\mu^{2}}{2 s^{2}}\right),
$$

where $\mu$ and $s$ are the mean and standard deviation in log-scale.

\section{B1 Implementation of the Adaptive Metropolis algorithm}

In order to enhance the efficiency of the MCMC algorithm and ease the problem of choosing an optimal proposal before the start of the MCMC simulation, we have implemented an Adaptive Metropolis (AM) algorithm (Haario et al., 2001) where a Gaussian proposal distribution is updated according to the statistics of the chain. Specifically, the covariance and mean of a multivariate Gaussian is recomputed at regular intervals.

In Section 6.3 we have chosen to define a multivariate Gaussian proposal per layer $l$. Each layerwise proposal is updated independently according to the history and correlation of the nodes within that layer. Since the nodal conductivity values can span several orders of magnitude, the Gaussian proposal is defined in log-scale and we evaluate its probability $q(\cdot \mid \cdot)$ in linear scale, i.e. a multivariate log-normal PDF centered at the current state $\mathbf{m}_{t-1}^{l}$ with covariance $\Sigma$ : 
$q\left(\mathbf{m}_{t}^{l} \mid \mathbf{m}_{t-1}^{l}\right)=\frac{1}{(2 \pi)^{\frac{n l a y}{2}}(\operatorname{det} \Sigma)^{\frac{1}{2}} \prod_{j=1}^{n l a y} m_{t}^{j}} \exp \left(-\frac{1}{2}\left(\ln \left(\mathbf{m}_{t}^{l}\right)-\ln \left(\mathbf{m}_{t-1}^{l}\right)\right)^{t} \Sigma^{-1}\left(\ln \left(\mathbf{m}_{t}^{\text {lay }}\right)-\ln \left(\mathbf{m}_{t-1}^{l}\right)\right)\right)$

where nlay is the number of nodes per layer and $\mathbf{m}^{l}$ is the proposed vector of nodes for layer $l$, i.e. conductivity values for all the nodes in layer $l$. 


\section{Supplementary Material “A Reduced Order Approach for Probabilistic Inversions of 3D Magnetotelluric Data I:}

\section{General Formulation"}

\section{BENCHMARKS MAX3D-G}

The problems presented and discussed in this section represent typical benchmarks found in the literature aiming at comparing solutions of the MT forward problem as well as to assess the computational efficiency of different algorithms. Here the particular interest is comparing our new implementation (hereafter referred to as Max3D-G) against the original Max3D algorithm with Domain Decomposition (hereafter referred to as Max3D-DDC) developed by Zyserman \& Santos (2000). The performance of these algorithms relative to some other popular codes is also discussed. Additional benchmarks of the original code, Max3D-DDC, can be found in Zyserman \& Santos (2000).

\subsection{DTM1 Model}

In 2008, a 3D-MT inversion and modeling workshop was held at the Dublin Institute for Advanced Studies with the aim of discussing and assessing the state of 3D modeling in magnetotellurics. In order to compare the forward and inversion solutions from different codes, several electrical conductivity models were proposed. The reader is referred to the review of this workshop by Miensopust et al. (2013) for details on the proposed models and obtained results. Of all the proposed models, the DTM1 model was specifically developed to show how different codes deal with strong resistivity contrasts. It consists of three blocks of different conductivity $(10,1$ and $10000 \Omega m$ ) inside an homogeneous 100 $\Omega m$ semi-space. Figure 1 shows a description of the blocks and a plan view of the model. The origin of coordinates is placed at the center of body 1 . Figure 2 shows two vertical slices of the model for different northing profiles.

In what follows, we compare the results and computation times for the DTM1 model for various 


\section{M.Constanza Manassero}

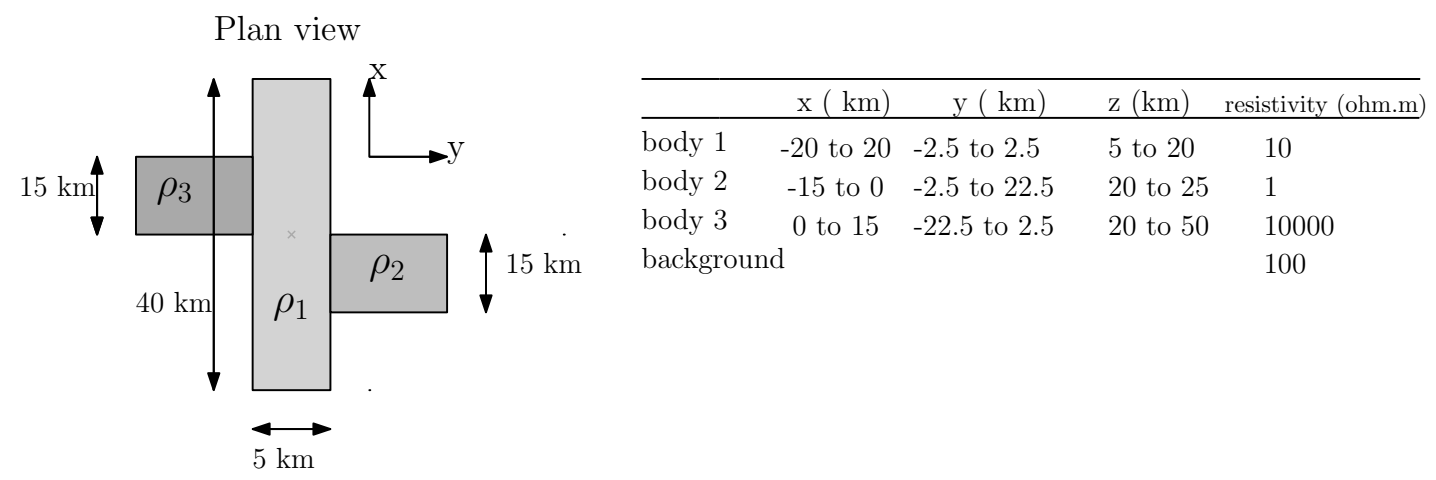

Figure 1. Plan view of DTM1 model. The symbol $\times$ indicates the origin of coordinates

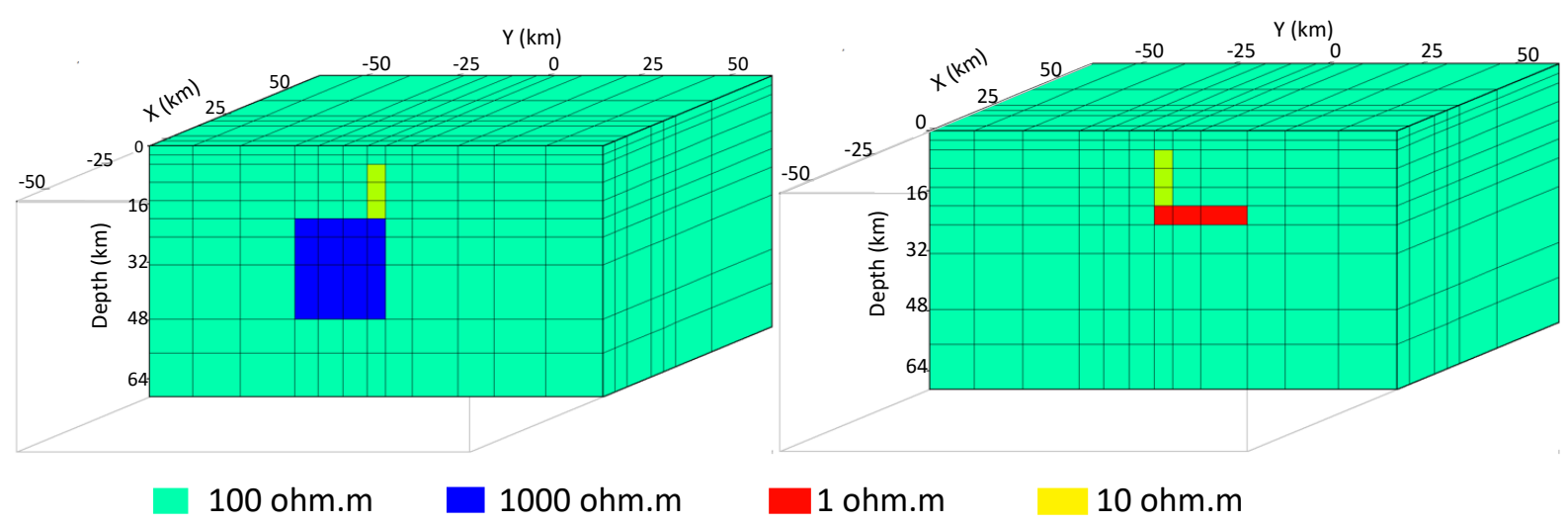

Figure 2. Different side views of the DTM1 model illustrating the three anomalies embedded in a 100 background.

codes including Max3D-DDC and Max3D-G. The results obtained with each code depend on the mathematical formulation, its numerical approximation (i.e., finite differences, integral equation or finite elements), the discretization of the domain, the coordinate system and how boundary conditions are implemented.

For our comparison we use the 3D forward responses provided by Prof. Alan Jones in his MTnet website ${ }^{\star}$ computed by different users of the following codes:

(1) The FE code of Myung Jin Nam (Nam et al., 2007) used by Nuree Han and Tae Jong Lee.

(2) WinGLink FD code (Mackie et al., 1994) used by Randy Mackie and Marion Miensopust.

(3) Wsinv3dmt FD code (Siripunvaraporn et al., 2002) used by Marion Miensopust.

(4) Mt3dinv FD code (Farquharson et al., 2002) used by Marion Miensopust.

* http://www.mtnet.info/main/ 
The forward responses were computed for a period range of $0.1 \mathrm{~s}$ to $10^{4} \mathrm{~s}$ at four periods per decade (21 periods) along 4 profiles with $5 \mathrm{~km}$ site spacing: profiles at $x=-15,0,15 \mathrm{~km}$ between $y=-37.5$ $\mathrm{km}$ and $y=37.5 \mathrm{~km}$ and a profile at $y=0$ between $x=-25 \mathrm{~km}$ and $x=25 \mathrm{~km}$. We follow the same procedure to compute our responses using Max3D-DDC and our new implementation Max3D-G.

For Max3D-DDC we used a model of $26 \times 26 \times 17$ elements plus one layer of air with resistivity $\rho=1 e^{15} \Omega m$. The layered 1D background is comprised of four layers and a semi-space of resistivity $\rho=100 \Omega m$. We computed the forward responses with 117 processors, obtaining the solution in 50.62 seconds. For Max3D-G and using the same model, we performed the computations with 2 processors per frequency to solve the linear system of equations using MUMPS and obtained the solution in 10.11 seconds.

Figures 4 and 3 show the apparent resistivity and phase of the impedance components computed with Max3D-DDC along two profiles located at $x=0$ and $y=0$ for $1000 \mathrm{~s}$ and along a profile located at $x=15 \mathrm{~km}$ for $1000 \mathrm{~s}$, respectively. Figures 6 and 5 show the apparent resistivity and phase computed with Max3D-G along the same profiles and periods. In all figures we include the results obtained with the codes (1)-(4). Table 7 extracted from Miensopust et al. (2013) lists the forward results obtained with the codes used at the workshop. With the aim of a comparison amongst the different approaches we have also included our results.

The results obtained with Max3D-DDC and Max3D-G are in excellent agreement with those predicted by the other tested algorithms. In particular, the results show that the responses for Max $3 D-G$ and Max3D-DDC are, in practice, identical, and that when using Max3D-G, we obtain time reductions of $80 \%$ with only $30 \%$ of the processors. We also observe a significant reduction in computational time when using Max3D-DDC and Max3D-G (Table 7) compared to the rest of the tested algorithms. This is because the use of absorbent boundary conditions (Sheen, 1997) and a secondary field formulation allow us to obtain accurate solutions while keeping the size of the numerical domain smaller than that needed in other formulations, and, therefore, reducing the computational time.

\subsection{Model 2}

Here we present a comparison of the forward responses predicted by ModEM (Egbert \& Kelbert, 2012), Max3D-DCC and our new implementation, Max3D-G.

The model used comprises three blocks of $2000 \Omega m, 5 \Omega m$ and $40 \Omega m$, respectively, embedded in an homogeneous semi-space of $100 \Omega \mathrm{m}$. Figure 8 describes the coordinate system, location and dimensions of the blocks.

Forward responses were calculated for a period range of $1 \mathrm{~s}$ to $10^{4} \mathrm{~s}$, with seven periods per decade 


\section{M.Constanza Manassero}
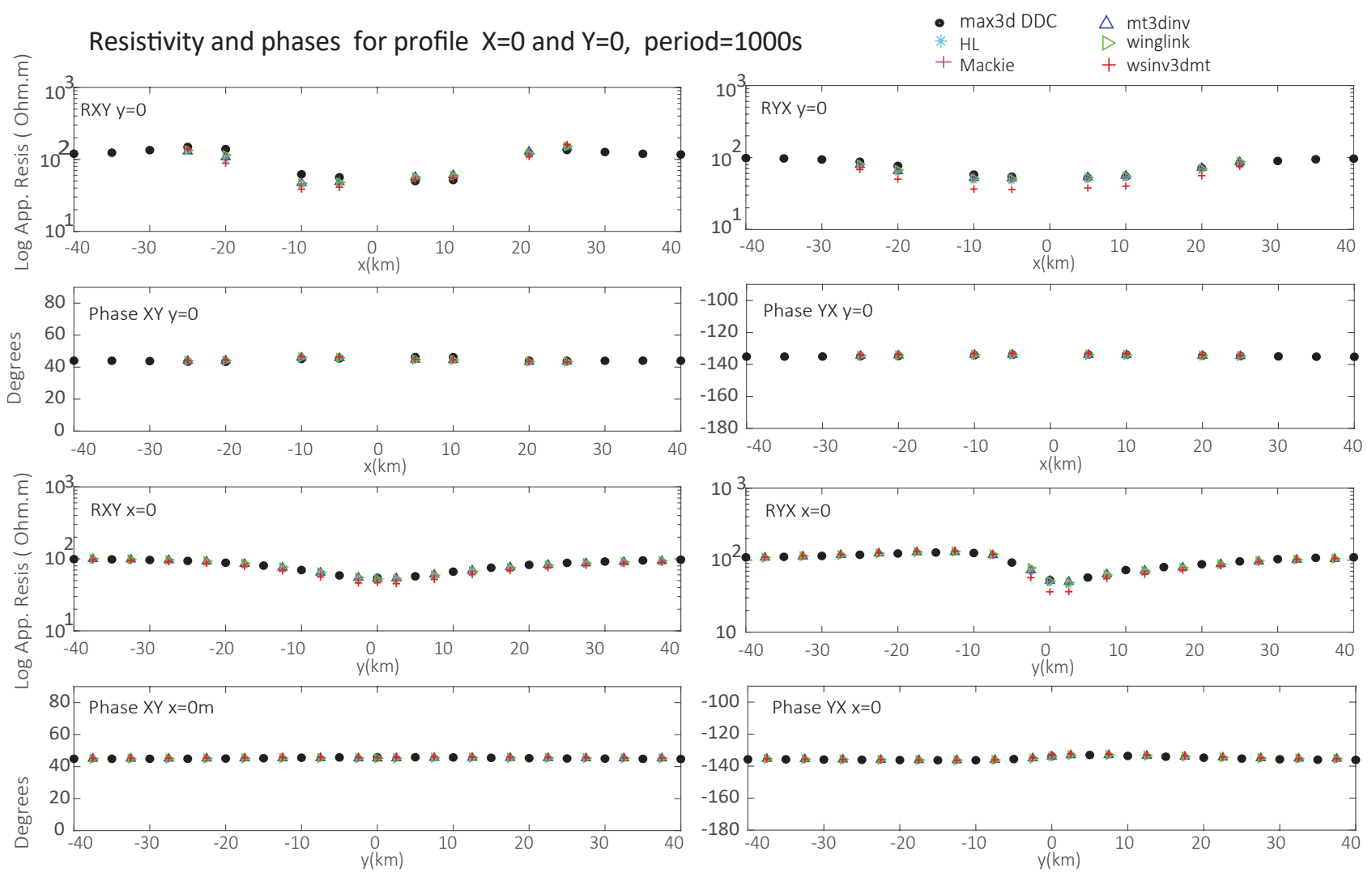

Figure 3. Apparent resistivity and phase derived from the off-diagonal elements of the impedance tensor at profiles $x=0$ and $y=0$ for 1000 s. We use Max3D-DDC and other codes referred to as: HL (Nam et al., 2007), Mackie (Mackie et al., 1994), winGLink (Mackie et al., 1994), wsinv3dmt (Siripunvaraporn et al., 2002) and mt3dinv (Farquharson et al., 2002).

( 29 periods), at 2500 sites located between $-125 \mathrm{~km}$ and $125 \mathrm{~km}$ in both directions ( $x$ and $y$ ). The spacing between stations was set to $5 \mathrm{~km}$. Figure 9 shows a side view of the model.

The numerical model used to compute the responses with ModEM was $1216 \mathrm{~km} \times 1216 \mathrm{~km} \times$ $1092 \mathrm{~km}$, discretized with $74 \times 74 \times 50$ cells. The computation was done using the control parameters of Table 1 .

\begin{tabular}{ll}
\hline Number of QMR iters per divergence correction & 40 \\
Maximum number of divergence correction calls & 20 \\
Maximum number of divergence correction iters & 100 \\
Misfit tolerance for EM forward solver & $1.0 e^{-7}$ \\
Misfit tolerance for EM adjoint solver & $1.0 e^{-7}$ \\
Misfit tolerance for divergence correction & $1.0 e^{-5}$ \\
\hline
\end{tabular}

Table 1. Control parameters used for the ModEM inversion 

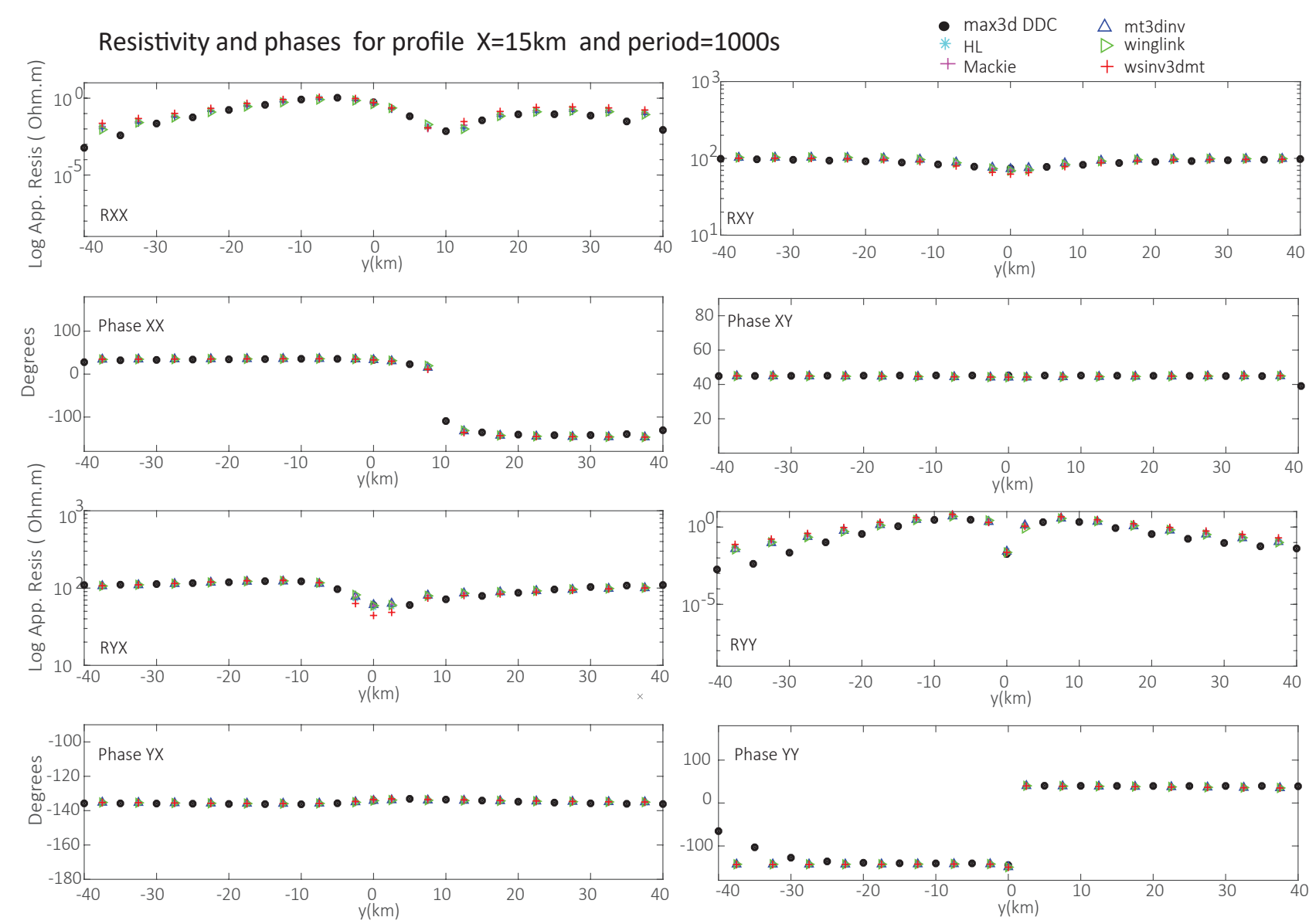

Figure 4. Apparent resistivity and phase derived from all elements of the impedance tensor at profile $x=15$ $\mathrm{km}$ and period of $1000 \mathrm{~s}$. We use Max3D-DDC and other codes referred to as: HL (Nam et al., 2007), Mackie (Mackie et al., 1994), winGLink (Mackie et al., 1994), wsinv3dmt (Siripunvaraporn et al., 2002) and mt3dinv (Farquharson et al., 2002).

The solution was obtained in 163 seconds using 50 processors Xeon(R) CPU E5-2680 @2.70GHz. The time increased to 198 seconds when using 64 processors.

In order to study the advantages of the absorbent boundary conditions in terms of the domain size and associated reduction in computational time, we used a model of considerably smaller size for both implementations of Max3D: $250 \mathrm{~km} \times 250 \mathrm{~km} \times 180 \mathrm{~km}$ and $50 \times 50 \times 40$ cells. The background model included one air layer of resistivity $\rho=1 e^{15} \Omega m$ and four 1D layers plus a semi-space of resistivity $\rho=100 \Omega m$. The computation for the 29 periods using Max3D-DCC took 665.8 seconds using 50 processors Xeon(R) CPU E5-2680 @2.70GHz, whereas Max3D-G solved the problem in 44 seconds using 12 processors per frequency and 158 seconds using 4 processors per frequency.

Figures 10 and 11 show a comparison of the apparent resistivity and phase derived from the offdiagonals components of the impedance tensor computed with Max3D-DCC and ModEM. The same 


\section{M.Constanza Manassero}
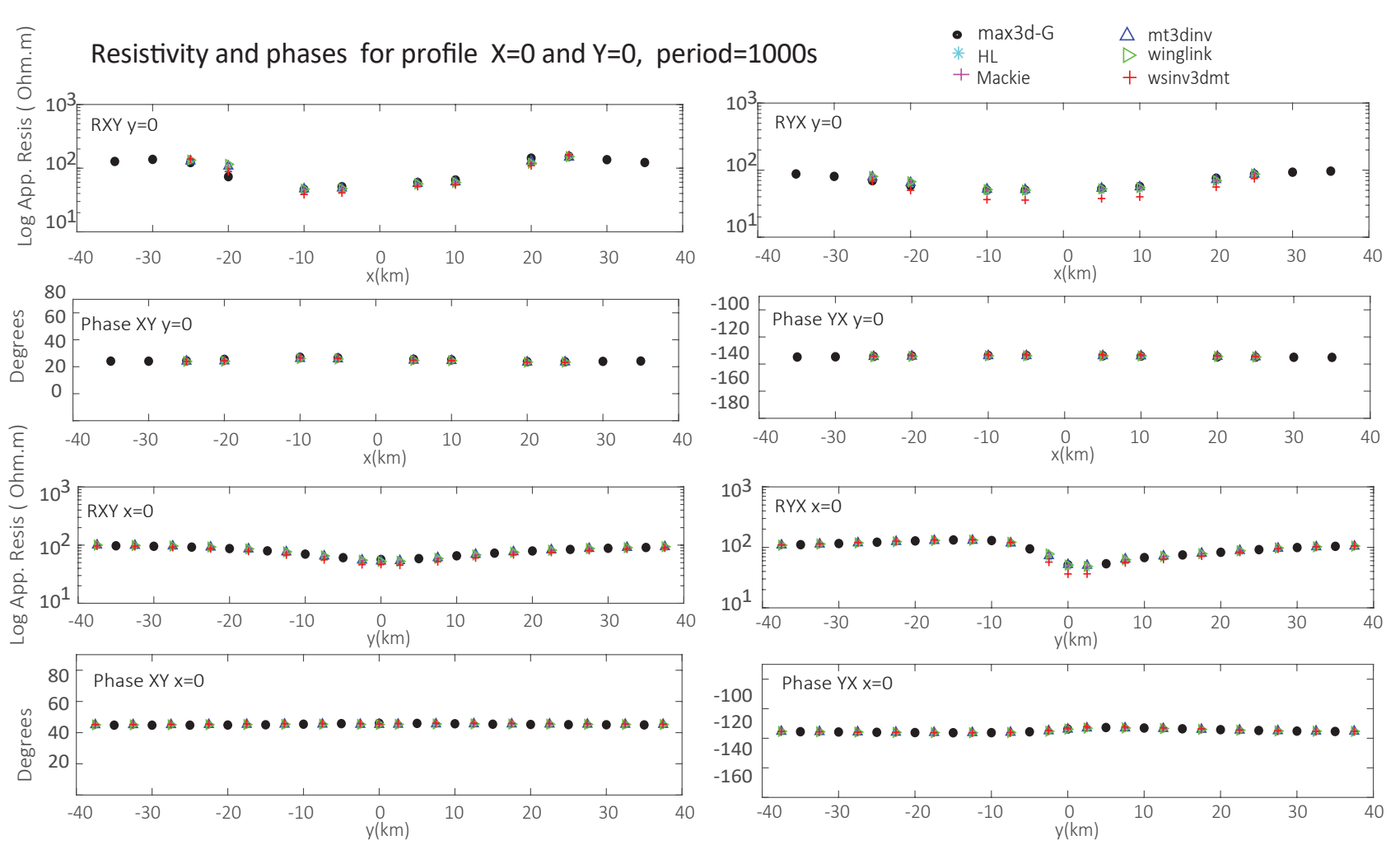

Figure 5. Apparent resistivity and phase derived from the off-diagonal elements of the impedance tensor at profiles $x=0$ and $y=0$ for $1000 \mathrm{~s}$. We use Max3D-G and other codes referred to as: $H L$ (Nam et al., 2007), Mackie (Mackie et al., 1994), winGLink (Mackie et al., 1994), wsinv3dmt (Siripunvaraporn et al., 2002) and mt3dinv (Farquharson et al., 2002).

comparison, but now between Max3D-G and ModEM, is shown in Figures 12 and 13. The responses are plotted for the 50 cells placed at the centre of the model between $-125 \mathrm{~km}$ to $125 \mathrm{~km}$ at $x=0$ and for all the 29 frequencies (vertical axis). The first frequency (top of y axis) is the period 1s and the 29 th (bottom of y axis) is the period $10^{4} \mathrm{~s}$.

The responses of Max3D-G and Max3D-DCC are, for all practical purposes, identical. The minor differences are mostly due to the iterative process of Max3D-DCC and associated convergence tolerance. The responses predicted by all three codes (Max3D-G, Max3D-DCC, ModEM) agree well with each other. Some minor differences are observed in the phase and apparent resistivity at periods higher than 100s (freq $n^{\circ} 15$ in the figures) and 1000s (freq $n^{\circ} 22$ in the figures), respectively, where ModEM shows higher values in the phase of $Z_{x y}$ and $Z_{y x}$ and lower apparent resistivity for the $Z_{x y}$ component. Both Max3D-G and Max3D-DCC, introduce a small boundary effect associated with the smaller size of the domain. This effect can be seen in the right and left border (cell $n^{\circ} 1$ and $n^{\circ} 50$ ) of panels (a) of Figs.10 and 11. It can be also noted that although the numerical domain used for ModEM was considerably larger than that used for Max3D a boundary effect seems to be present in 

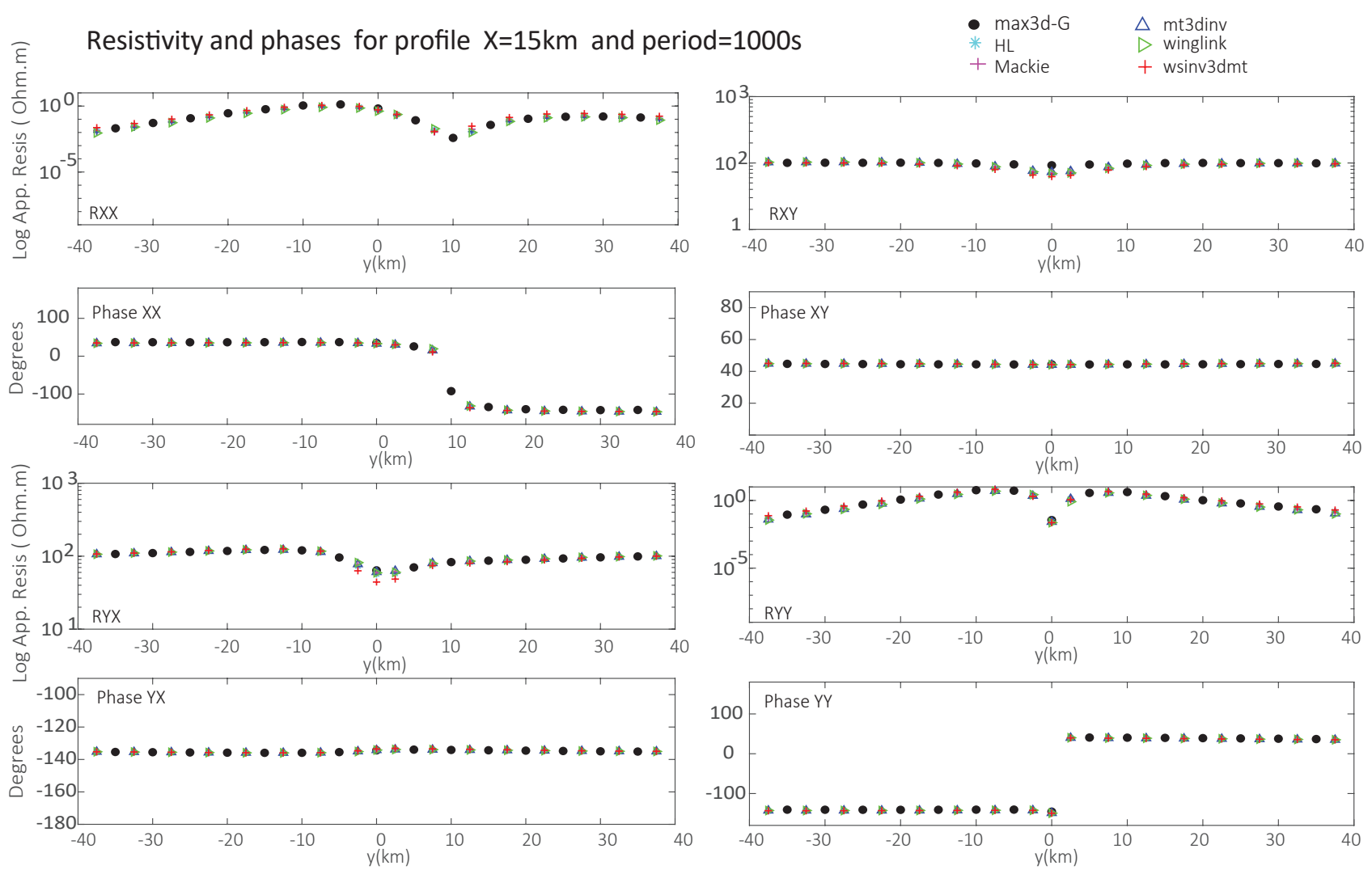

Figure 6. Apparent resistivity and phase derived from all elements of the impedance tensor at profile $x=15$ $\mathrm{km}$ for 1000s. We use Max3D-G and other codes referred to as: $H L$ (Nam et al., 2007), Mackie (Mackie et al., 1994), winGLink (Mackie et al., 1994), wsinv3dmt (Siripunvaraporn et al., 2002) and mt3dinv (Farquharson et al., 2002).

the ModEM responses for low frequencies. This possible boundary effect can be observed for period 10000s in apparent resistivities (freq $n^{\circ} 29$, bottom of panels (c) and (d) of Figs.10 and 12) and for periods higher than 3727s (freq $n^{\circ} 27$, bottom of panels (c) and (d) of Figs.11 and 13 ). It is unclear if this effect may be the reason for the differences observed at high periods or if ModEM exhibits higher sensitivity to the deeper conductive block than Max3D-DCC and Max3D-G.

In an attempt to clarify these differences, Figure 14 shows the subtraction of apparent resistivity and phase computed with Max3D-G from the responses computed with ModEM. The differences are less than $6 \Omega m$ in apparent resistivity and 1 degree in phase, displaying the aforementioned sensitivity of ModEM. The small numerical boundary effect introduced by Max3D-G and Max3D-DCC, and the possible boundary effect in the ModEM responses are also notable. Given the similar results between Max3D-G and ModEM together with the considerable computational time difference (163 seconds against 44 seconds) and the excellent performance of the absorbent boundaries conditions we adopt our implementation Max3D-G for the work that follows and our probabilistic inversion framework. 


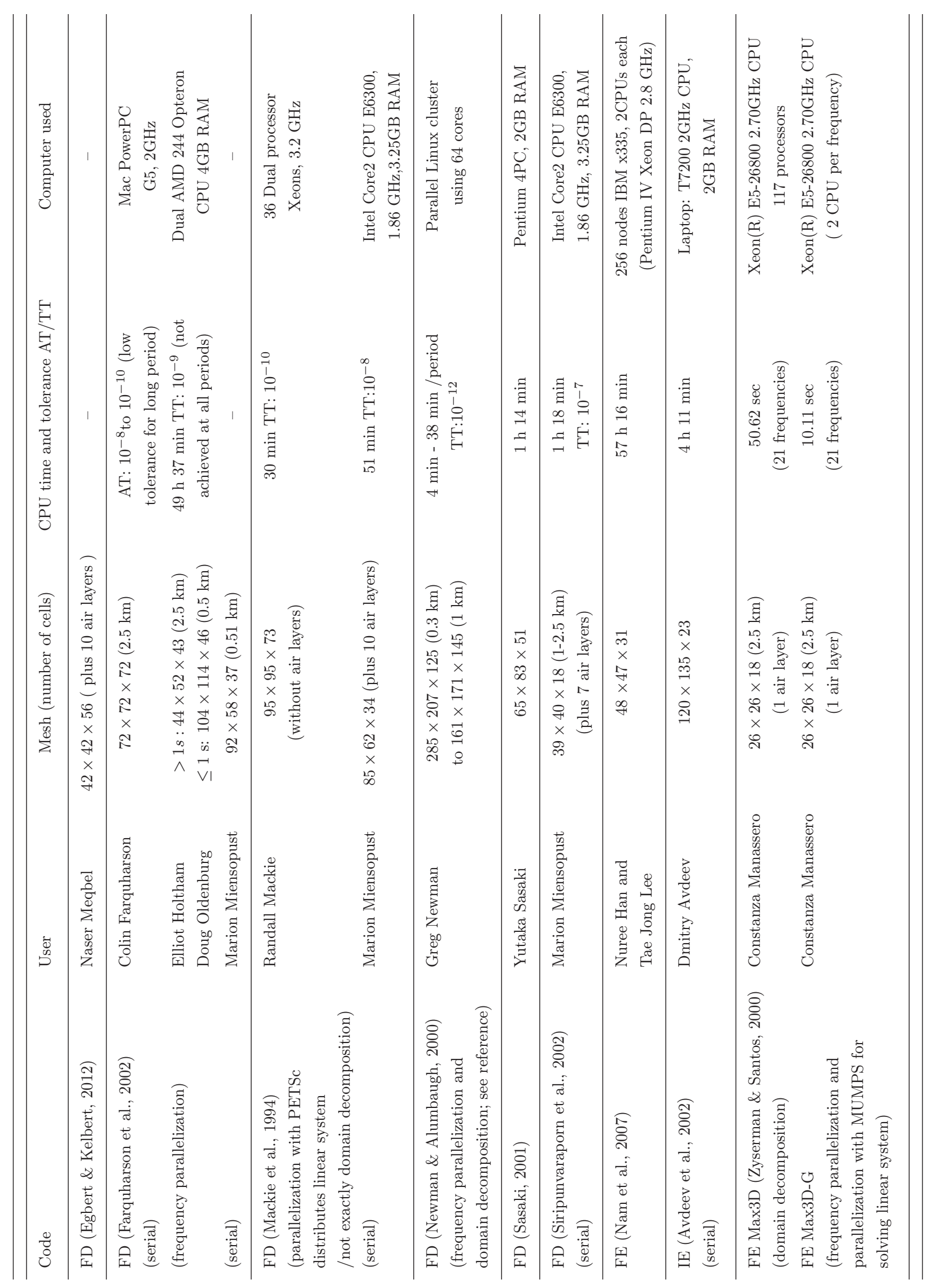

Figure 7. List of DTM1 forward results extracted from Miensopust et al. (2013) and modified to include our results. The table specifies: code and its type (FD, FE or IE), user, mesh and in parentheses (if available) the lateral width of the cells at the centre of the model, required CPU time (and target tolerance TT or achieved tolerance AT) and computer used. 


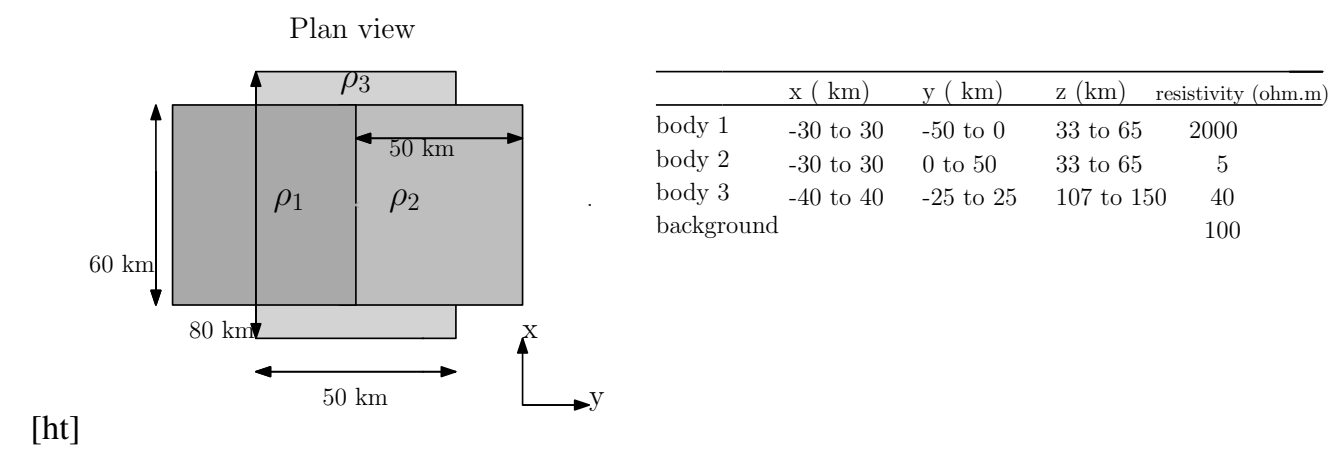

Figure 8. Plan view Model 2. The symbol $\times$ indicates the origin of coordinates.

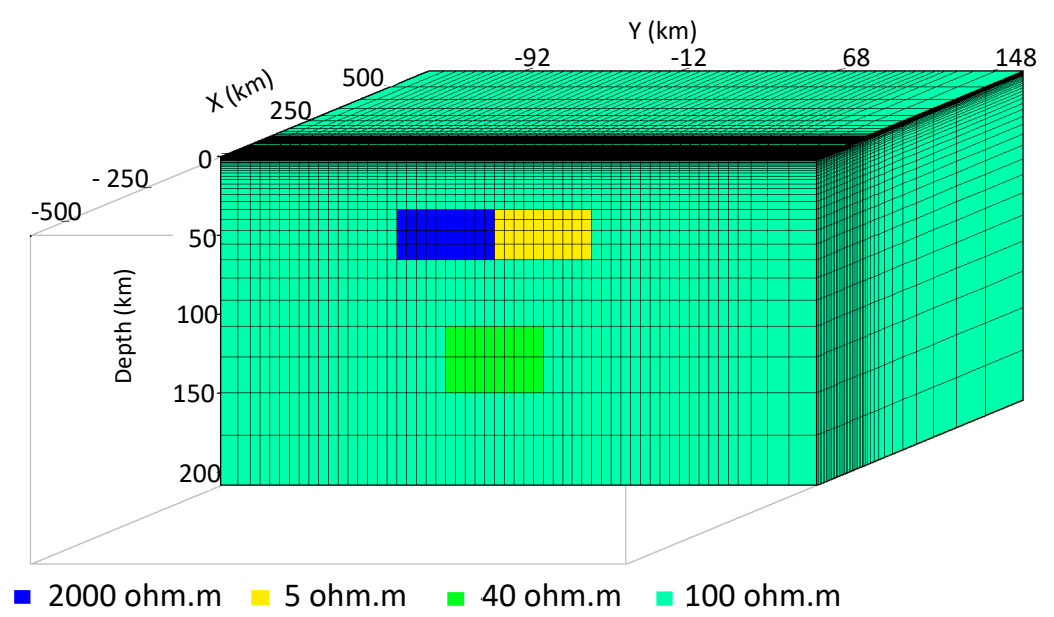

Figure 9. Side view Model 2 showing three anomalies embedded in a 100 background.

\section{AN ILLUSTRATIVE APPLICATION OF THE RB+MCMC ALGORITHM: 1D HEAT-TRANSFER PROBLEM}

In this section, we illustrate the basic features of the RB+MCMC algorithm with a simple and familiar 1D heat transfer problem. This allows us to compute the exact posterior PDFs using high-fidelity numerical models that we can use to compare with the approximations from our method.

\subsection{Model setup}

The forward problem involves the calculation of 1D geotherms for a two-layer (crust plus lithospheric mantle) lithospheric model by solving the 1D steady-state equation 


\section{M.Constanza Manassero}
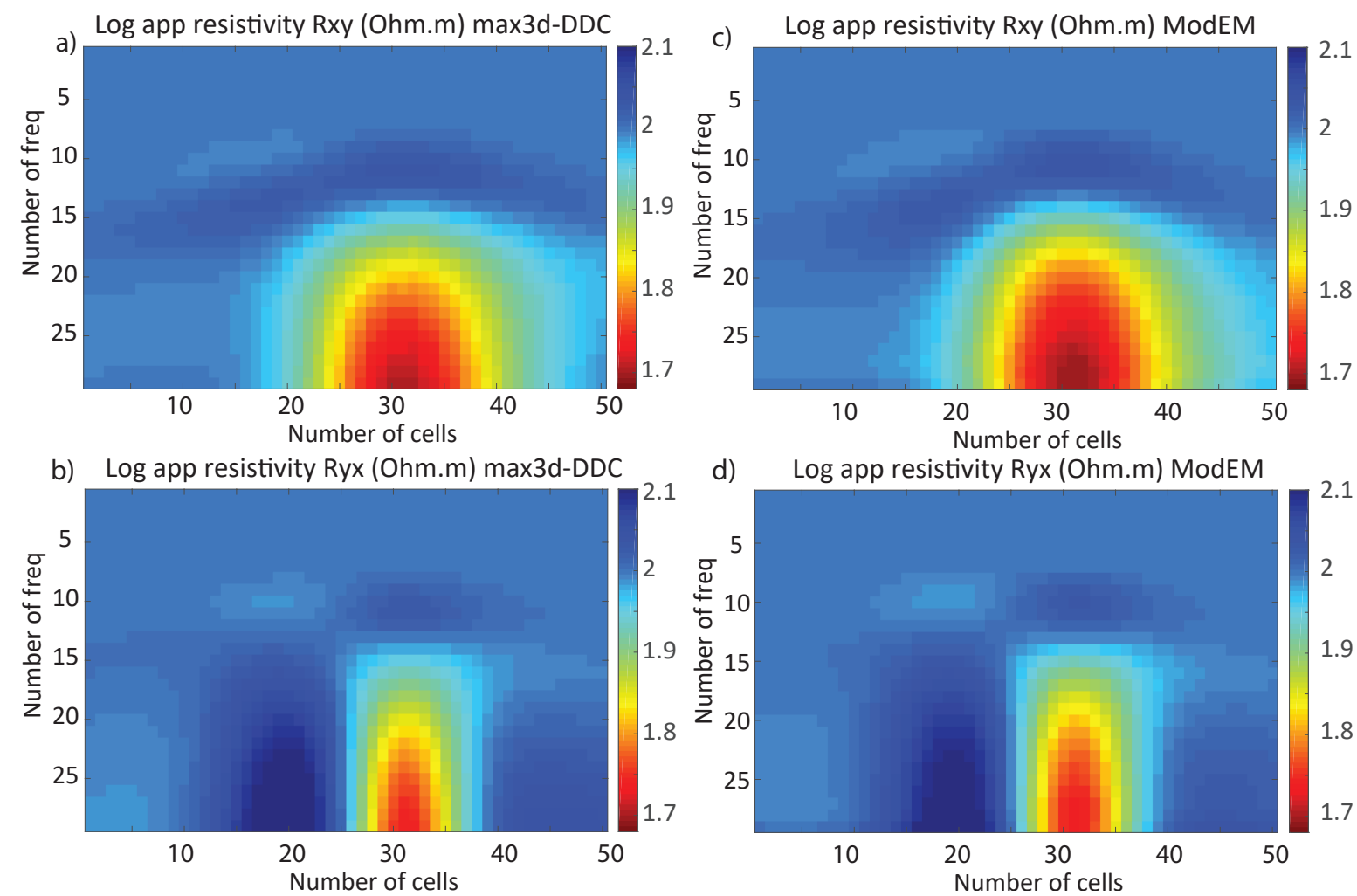

Figure 10. Apparent resistivity derived from the off-diagonals components of the impedance tensor computed with (a)-(b) Max3D-DDC and (c)-(d) ModEM. (a)-(d) Plots for 50 cells between $-125 \mathrm{~km}$ to $125 \mathrm{~km}$ at $x=0$ and for 29 frequencies (vertical axes) between $1 \mathrm{~s}$ and $10^{4} \mathrm{~s}$.
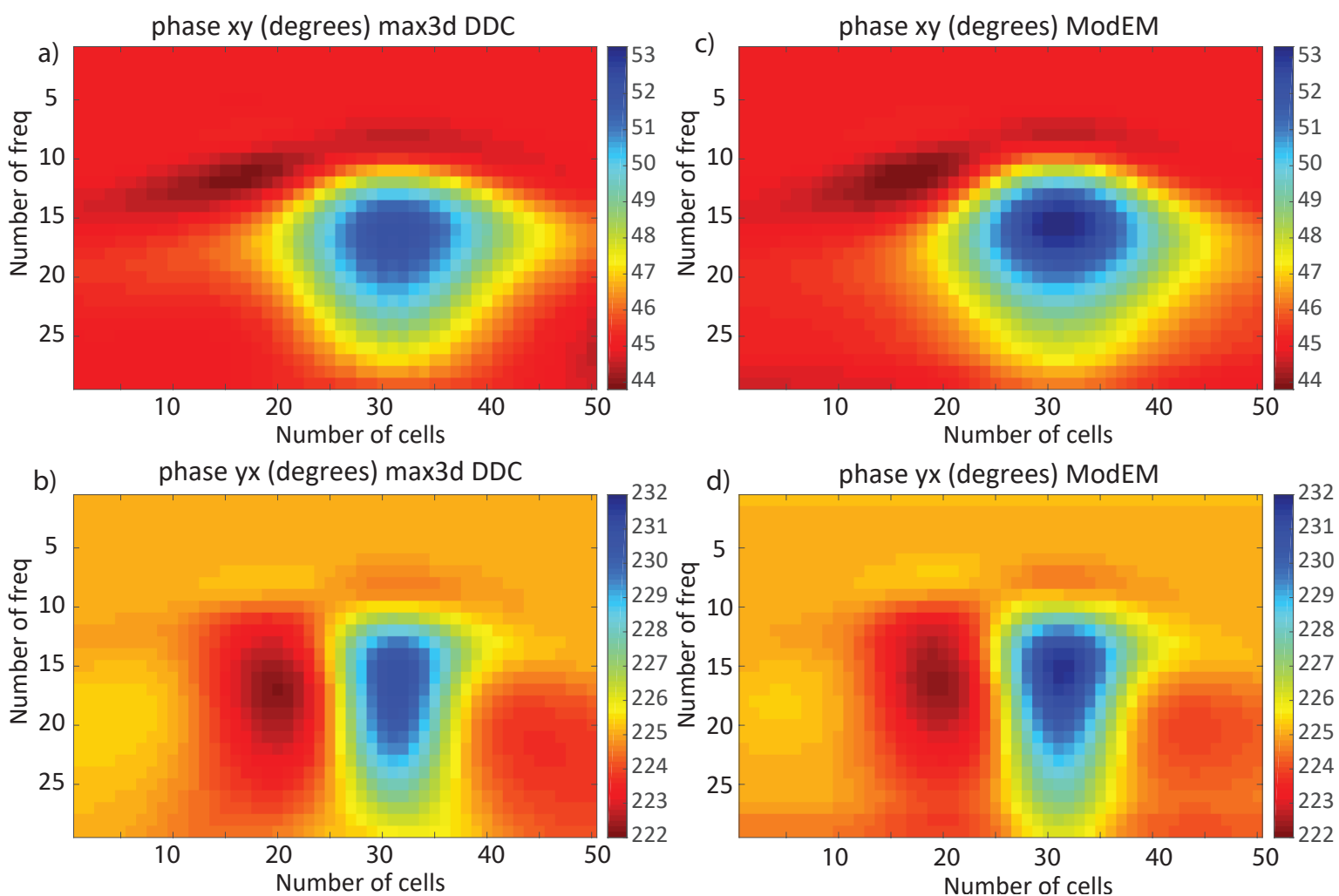

Figure 11. Apparent phase derived from the off-diagonal components of the impedance tensor computed with (a)-(b) Max3D-DDC and (c)-(d) ModEM. (a)-(d) Plots for 50 cells between $-125 \mathrm{~km}$ to $125 \mathrm{~km}$ at $x=0$ and for 29 frequencies (vertical axes) between $1 \mathrm{~s}$ and $10^{4} \mathrm{~s}$. 

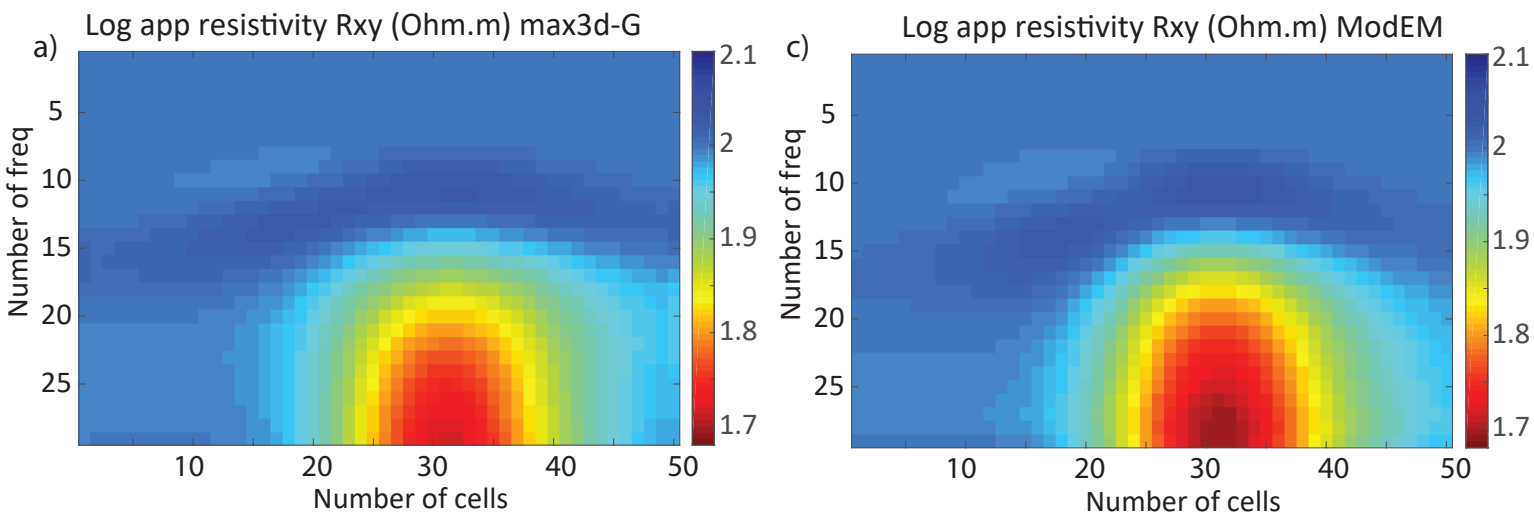

b) Log app resistivity Ryx (Ohm.m) max3d-G
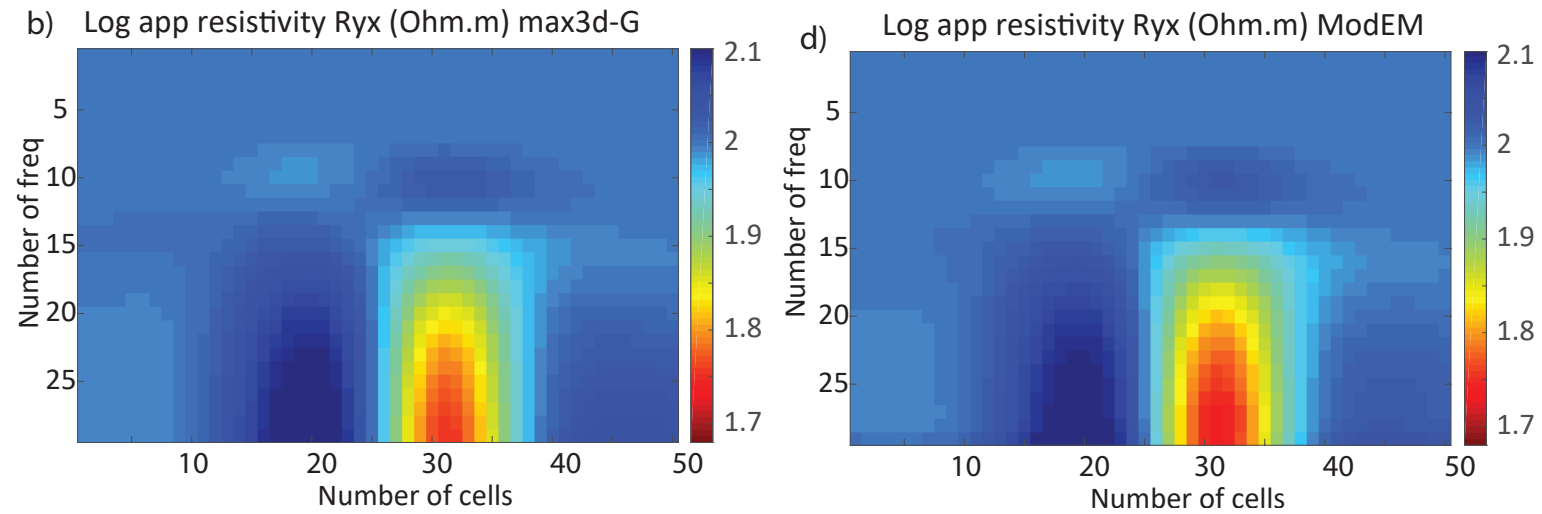

Figure 12. Apparent resistivity derived from the off-diagonal components of the impedance tensor computed with (a)-(b) Max3D-G and (c)-(d) ModEM. (a)-(d) Plots for 50 cells between $-125 \mathrm{~km}$ to $125 \mathrm{~km}$ at $x=0$ and for 29 frequencies (vertical axes) between $1 \mathrm{~s}$ and $10^{4} \mathrm{~s}$.
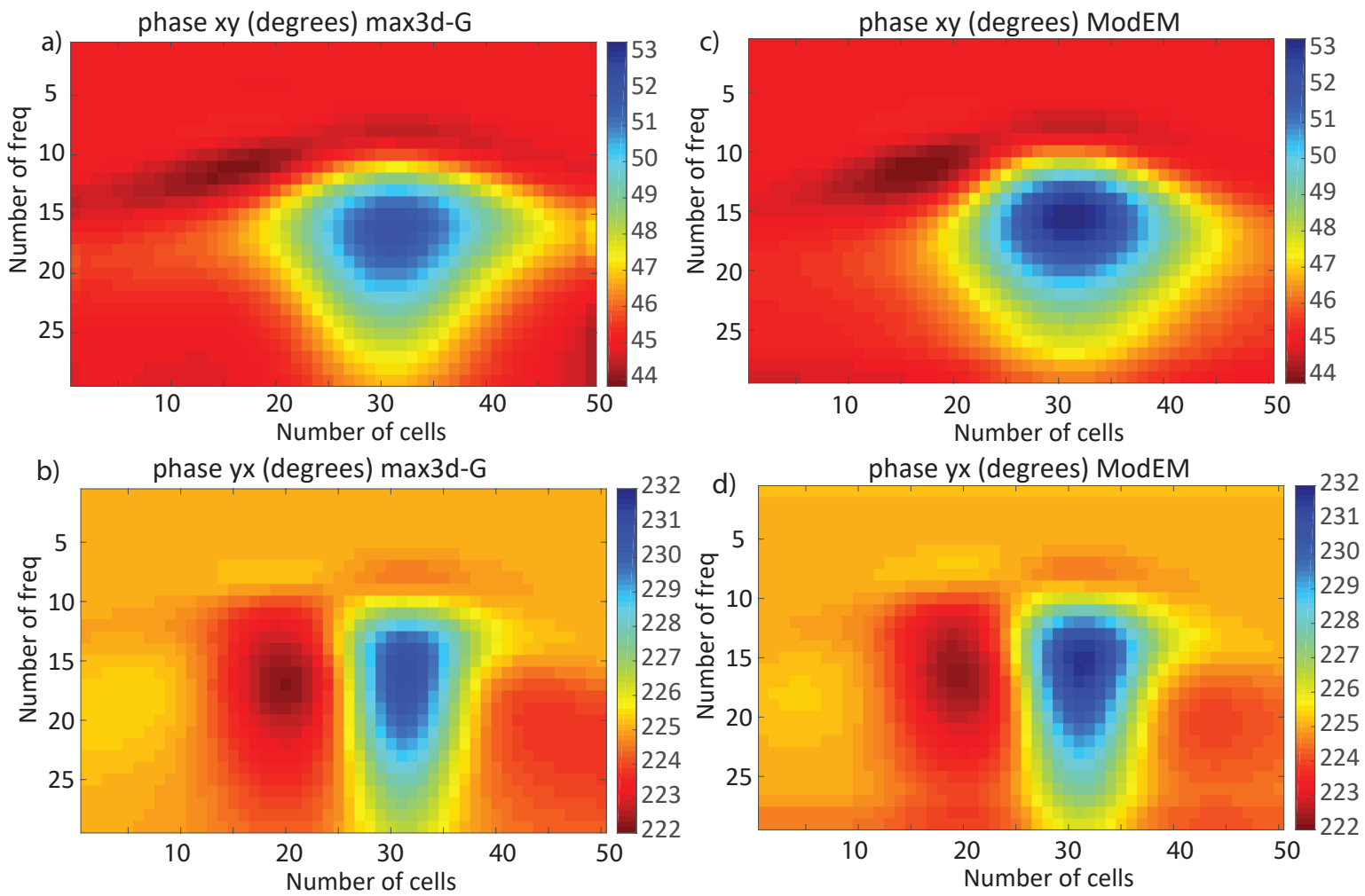

Figure 13. Apparent phase derived from the off-diagonals components of the impedance tensor computed with (a)-(b) Max3D-G and (c)-(d) ModEM. (a)-(d) Plots for 50 cells between $-125 \mathrm{~km}$ to $125 \mathrm{~km}$ at $x=0$ and for 29 frequencies (vertical axes) between $1 \mathrm{~s}$ and $10^{4} \mathrm{~s}$. 

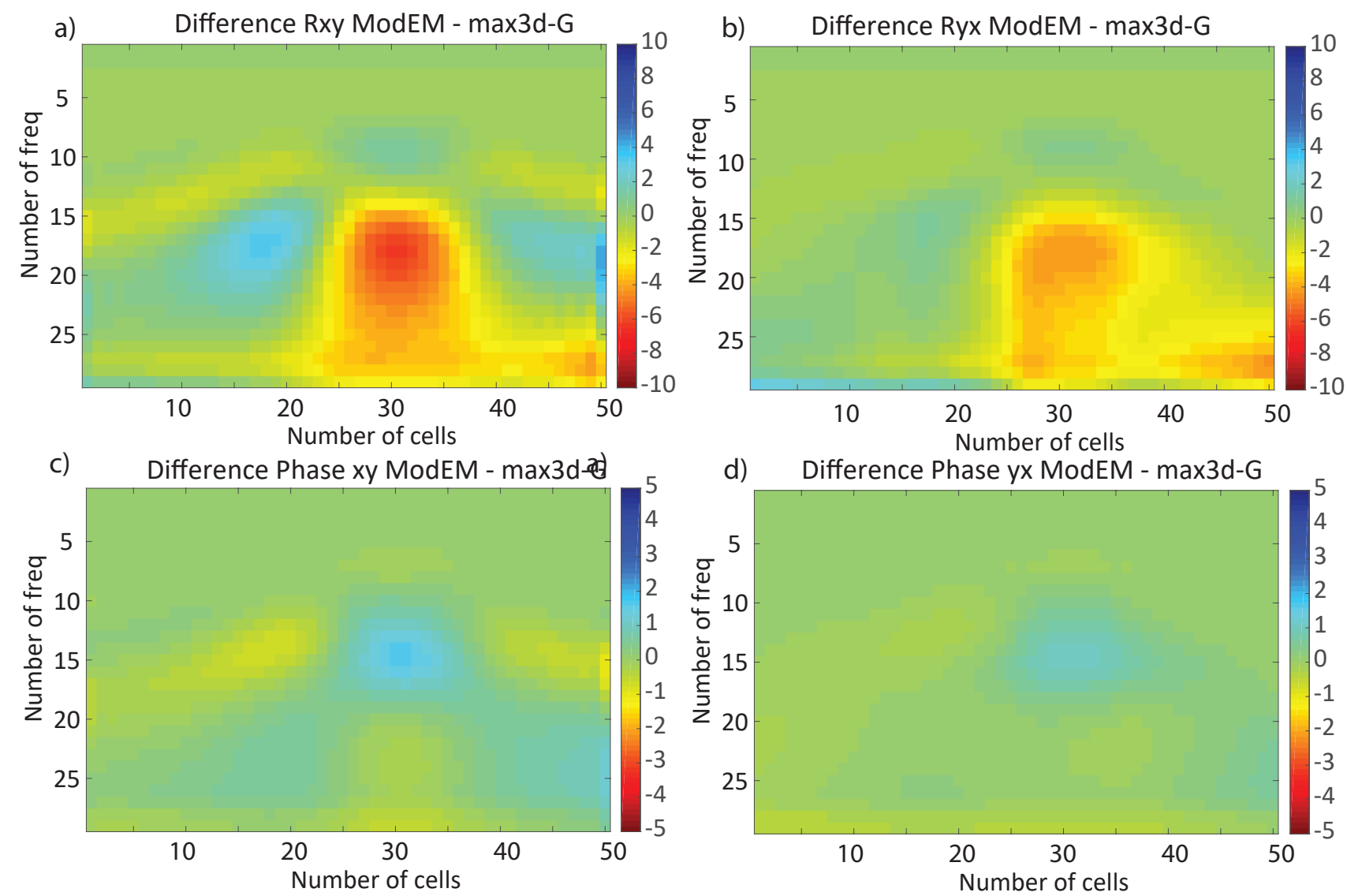

Figure 14. Difference (ModEM - Max3D-G) for (a)-(b) apparent resistivity and (c)-(d) phase derived from the off-diagonals components of the impedance tensor.

$$
-\frac{d}{d x}\left(k(x) \frac{d T}{d x}\right)=f(x)
$$

where the functions $T(x), k(x)$ and $f(x)$ are, respectively, temperature, thermal conductivity and heat source over an interval $[a, b]$. The boundary conditions are temperature at the surface $T(0 \mathrm{~km})=T_{s}$ and at the lithosphere-asthenosphere boundary $(\mathrm{LAB}) T(150 \mathrm{~km})=T_{l a b}$.

The synthetic data is the heat flux at the surface of the model $(Q s)$ and the unknown parameters are crustal thickness and radioactive heat production (RHP) per unit volume within the crust. The forward problem is solved with the Finite Element method using piecewise linear elements which can be found in:

https ://people.sc.fsu.edu/ jburkardt/m_src/fem1d_heat_steady/fem1d_heat_steady.html.

The synthetic data used in the inversion was obtained for a RHP $=1 e^{-6} \mathrm{~W} / \mathrm{m}^{3}$, Moho depth of $30 \mathrm{~km}$ and the constant values shown in Table 2. The resulting $Q s$ for these model parameters is 53.25 $m W / m^{2}$. We assumed uncorrelated Gaussian errors with a small standard deviation of $1 \mathrm{~mW} / \mathrm{m}^{2}$. 


\begin{tabular}{|l|l|}
\hline Surface depth & $0 \mathrm{~km}$ \\
Surface temperature & $10^{\circ} \mathrm{C}$ \\
LAB depth & $150 \mathrm{~km}$ \\
LAB temperature & $1300^{\circ} \mathrm{C}$ \\
Crust thermal conductivity & $2.6 \mathrm{~W} / \mathrm{mC}$ \\
Mantle thermal conductivity & $3.4 \mathrm{~W} / \mathrm{mC}$ \\
Mantle heat production & $2 e^{-8} \mathrm{~W} / \mathrm{m}^{3}$ \\
\hline
\end{tabular}

Table 2. Constant values assumed during the 1D Heat-Transfer inversion.

\subsection{Inversion Results}

In order to assess the performance of the $\mathrm{RB}+\mathrm{MCMC}$ algorithm, we ran MCMC inversions using full FE solutions (control/true solution) for all MCMC steps as well as our RB+MCMC algorithm setting different RB tolerances. In all cases, we use a uniform prior defined between 10 and $120 \mathrm{~km}$ for Moho depth and between $5 e^{-7}$ and $3.5 e^{-6} \mathrm{~W} / \mathrm{m}^{3}$ for the crustal RHP. The initial model (i.e. starting point in the chain) was located at $50 \mathrm{~km}-3.5 e^{-6} \mathrm{~W} / \mathrm{m}^{3}$. An independence sampler algorithm was used during the inversion (within the bounds given by the prior distribution).

The posterior joint and marginal PDFs obtained after 4,000,000 simulations are shown in Figures 15. The joint distribution exhibits the expected trade-off between crustal thickness and RHP. This highly correlated PDF results from the fact that surface heat flow is mostly sensitive to the amount of heat created inside the model (i.e. to the product of the parameters) and cannot distinguish between these two parameters. We deliberately chose this distribution as it is very challenging for any inversion algorithm. The joint and marginal posterior PDFs of both parameters (moho and $\mathrm{CPH}$ ) obtained using the $\mathrm{RB}+\mathrm{MCMC}$ algorithm with $\beta=1 e^{-6}$ (Fig.15.b) are identical to the posterior PDFs obtained with the full FE MCMC (Fig.15.a). The total number of bases computed during the 4,000,000 simulations of the RB+MCMC inversion was 40. This number is drastically smaller than the dimensions involved in 4,000,000 FE simulations in the original FE+MCMC inversion, resulting in an efficiency gain of $>$ $99 \%$.

Panels (c)-(f) of Fig.15 show the results for $\beta=1 e^{-5}, \beta=6 e^{-5}, \beta=1 e^{-4}$ and $\beta=1 e^{-3}$ with 29, 3, 2 and 1 bases, respectively. Reducing the number of bases to $29\left(\beta=1 e^{-5}\right)$ still produces good representations of the true distribution, although some small differences can be seen in the region around RHP $=-5.5$. Even in the drastic case of only 3 bases $\left(\beta=6 e^{-5}\right)$, the high probability regions are still identified, but the PDFs exhibits a clear bias.

The above numerical example clearly demonstrate the effectiveness of our algorithm with an efficiency gain of $>99 \%$ in all cases. An important but expected observation is that the performance of 


\section{M.Constanza Manassero}

the $\mathrm{RB}+\mathrm{MCMC}$ algorithm depends on the choice of the tolerance $\beta$. This tolerance is problem dependent and ultimately controls the creation of the bases and the bias between the approximated and the exact PDF. 


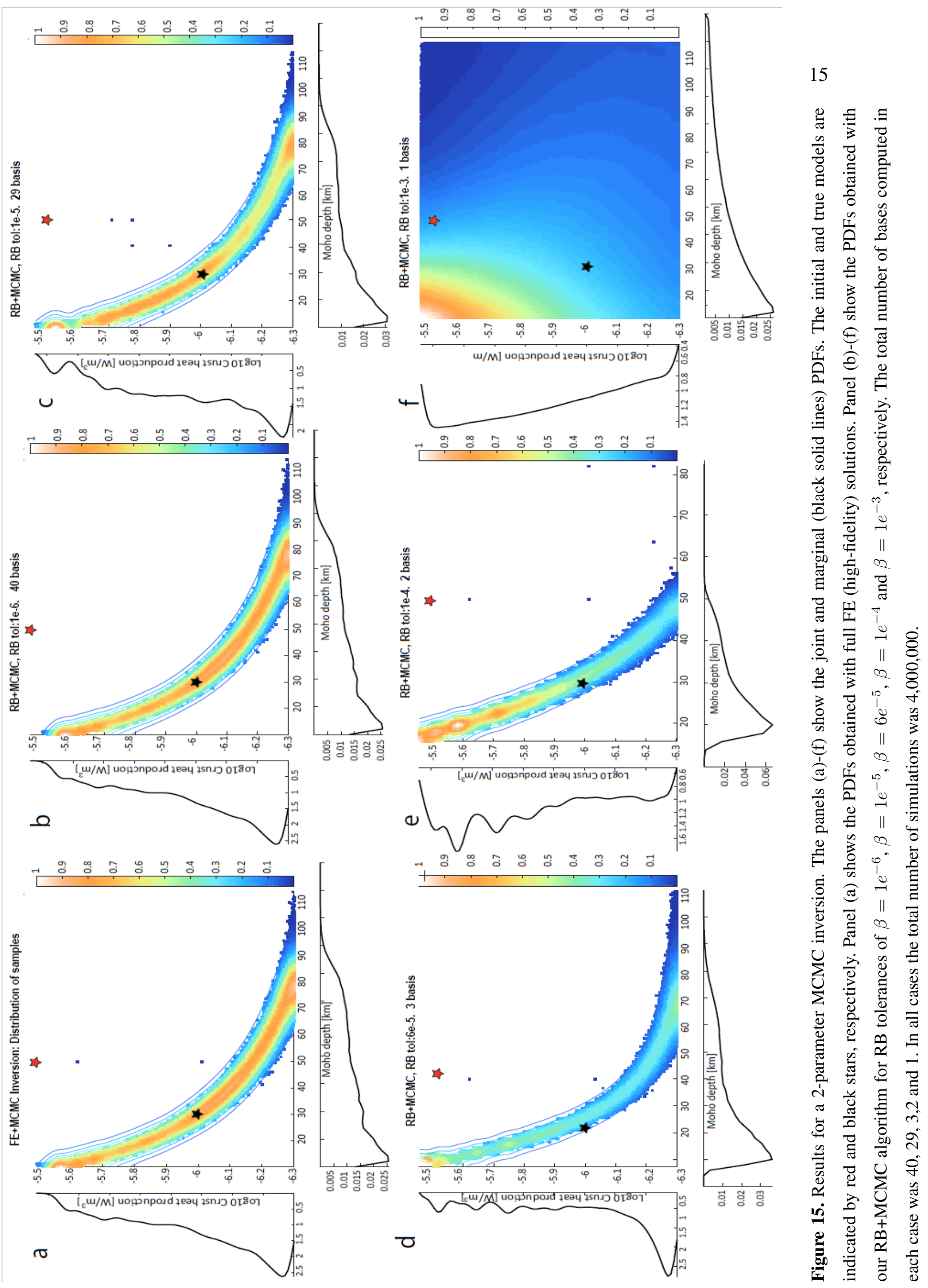




\section{M.Constanza Manassero}

\section{NUMERICAL EXAMPLE 1: ADDITIONAL INVERSION RESULTS}

This section shows additional results of the $\mathrm{RB}+\mathrm{MCMC}$ inversion of the large-scale lithospheric model presented Section 6.2. 


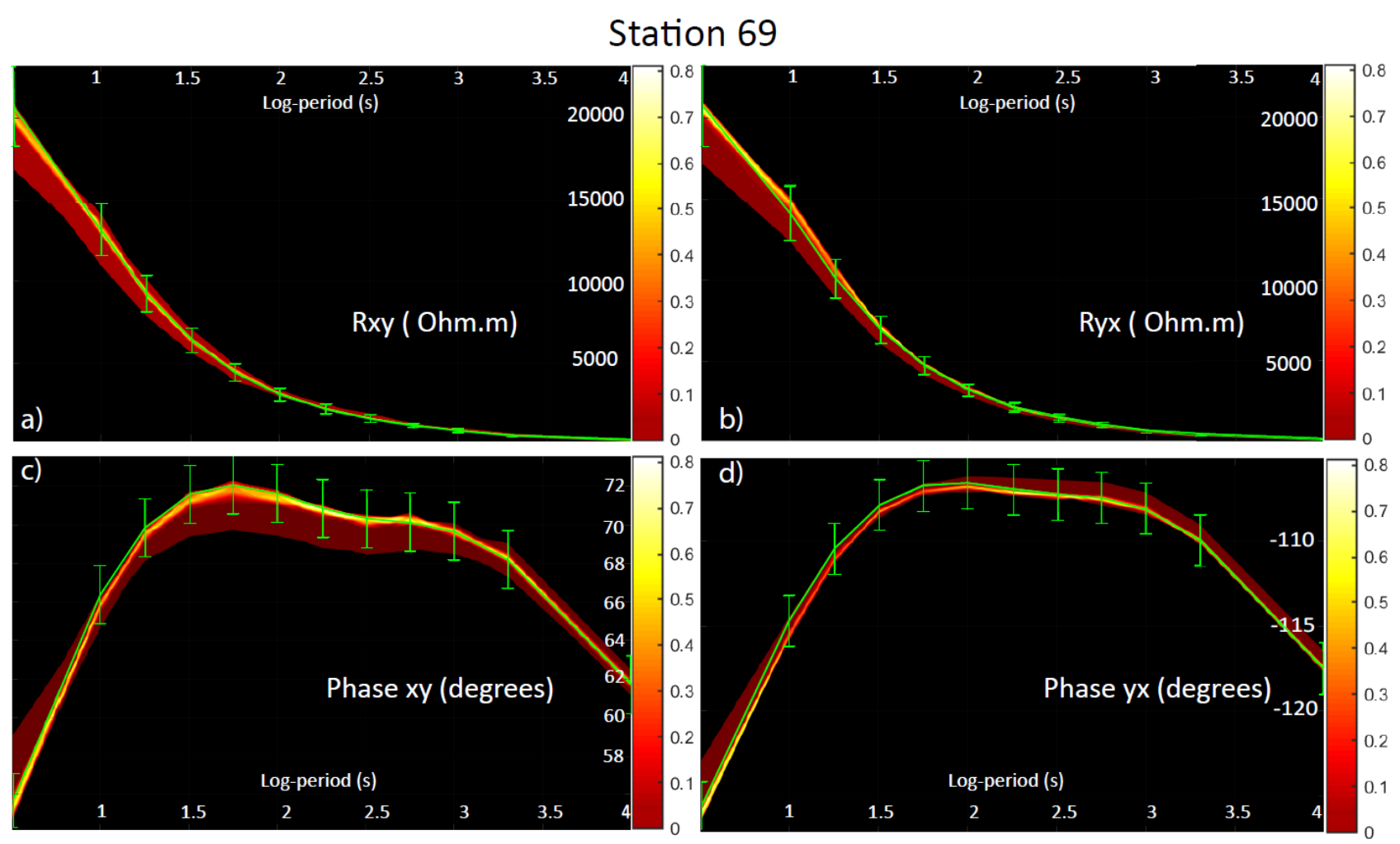

Figure 16. Posterior PDFs of data for station 69. Synthetic data and error bars are plotted in green. (a)-(b) Posterior PDFs of the off-diagonal apparent resistivity. (c)-(d) Posterior PDFs of the off-diagonal apparent phases.

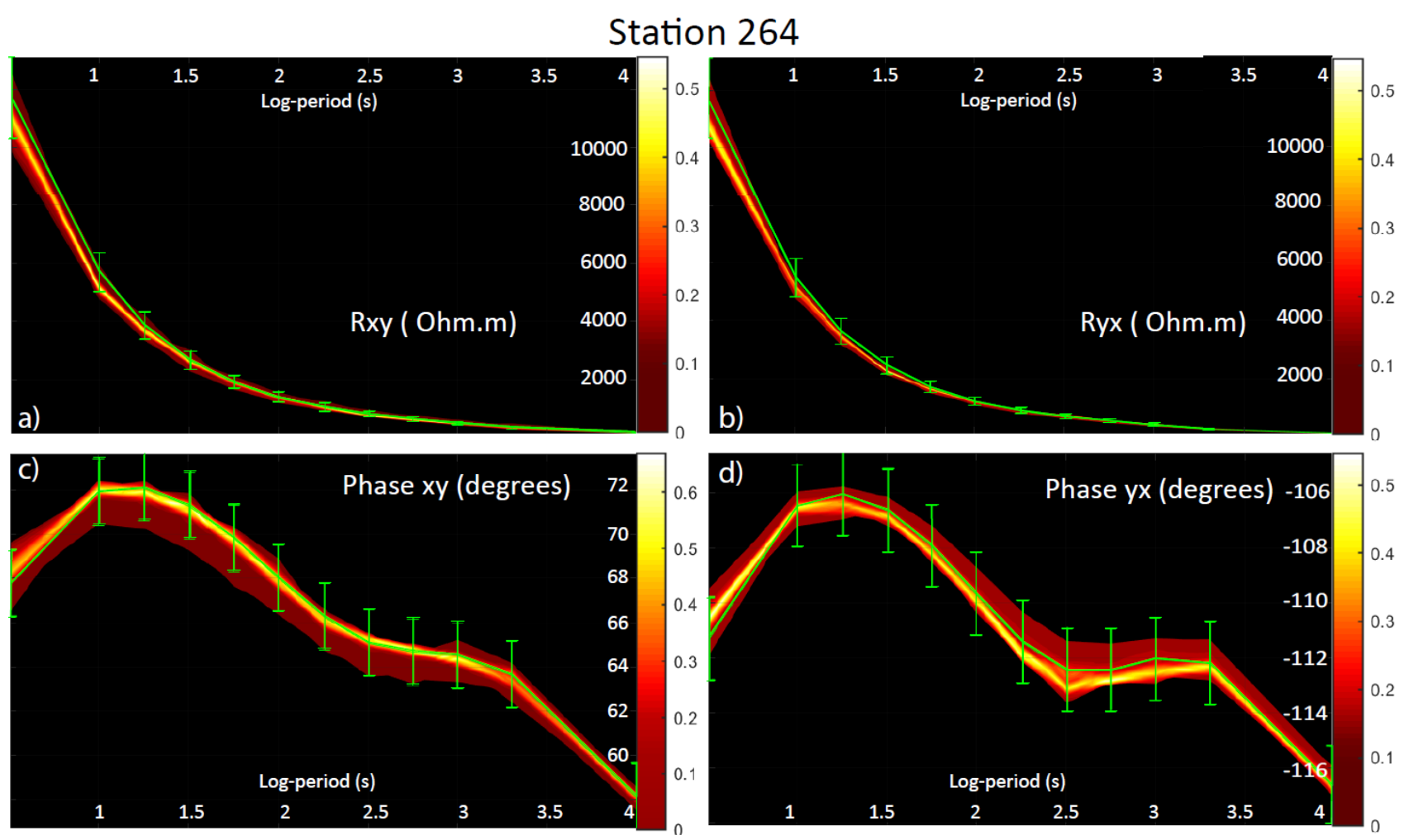

Figure 17. Posterior PDFs of data for station 264. Synthetic data and error bars are plotted in green. (a)-(b) Posterior PDFs of the off-diagonal apparent resistivity. (c)-(d) Posterior PDFs of the off-diagonal apparent phases. 

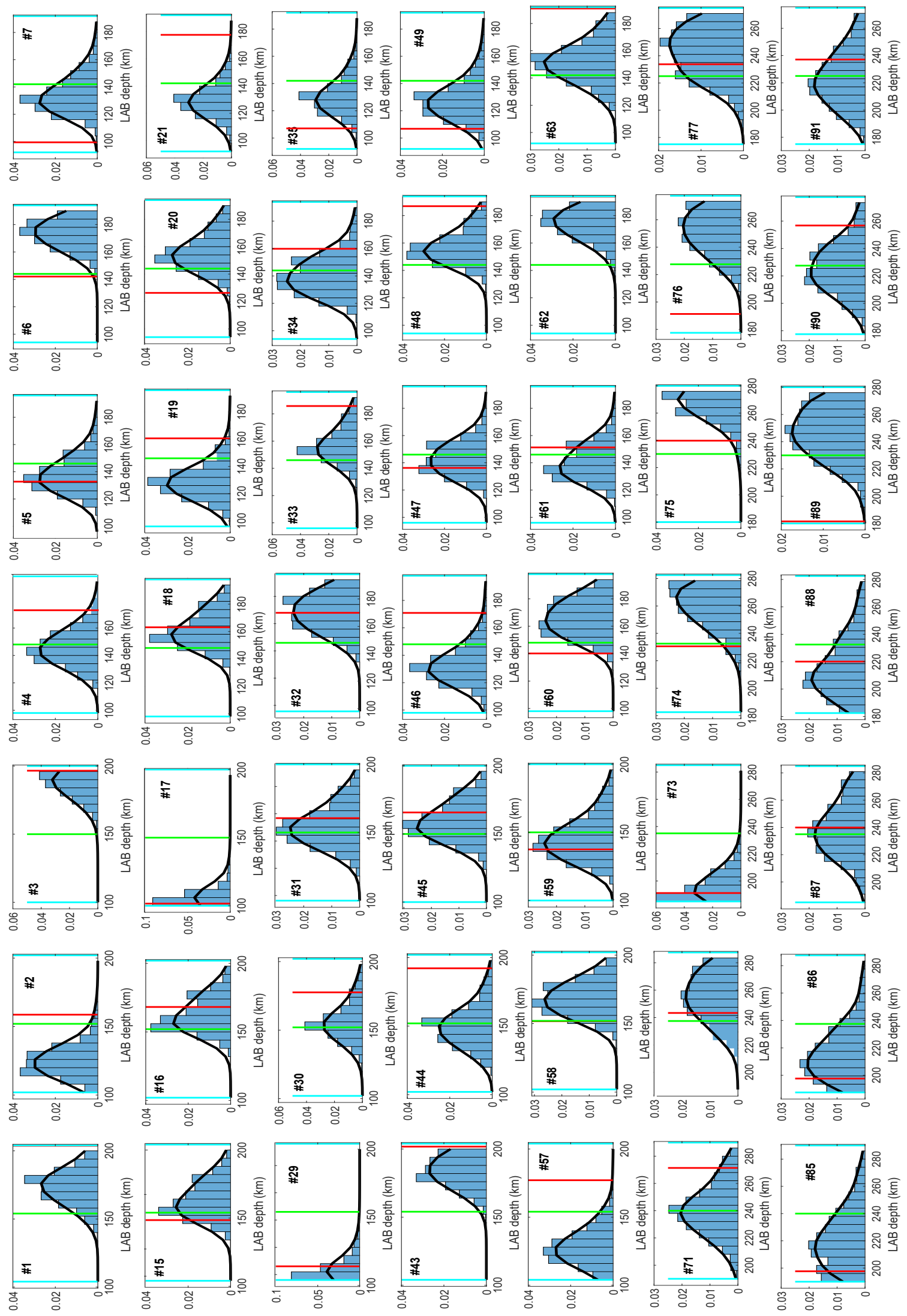

Figure 18. Marginal posterior PDFs (blue bars) and best fitting distributions (black lines) of 49 of the 196 model parameters obtained after 2,500,000 RB+MCMC simulations. The real value, starting value and prior bounds of each parameter are shown in green, red, and light blue vertical lines, respectively. The position of each PDF in the figure corresponds with the location of the 49 columns at the south-west corner of the model (Fig. 1.b in main text). 

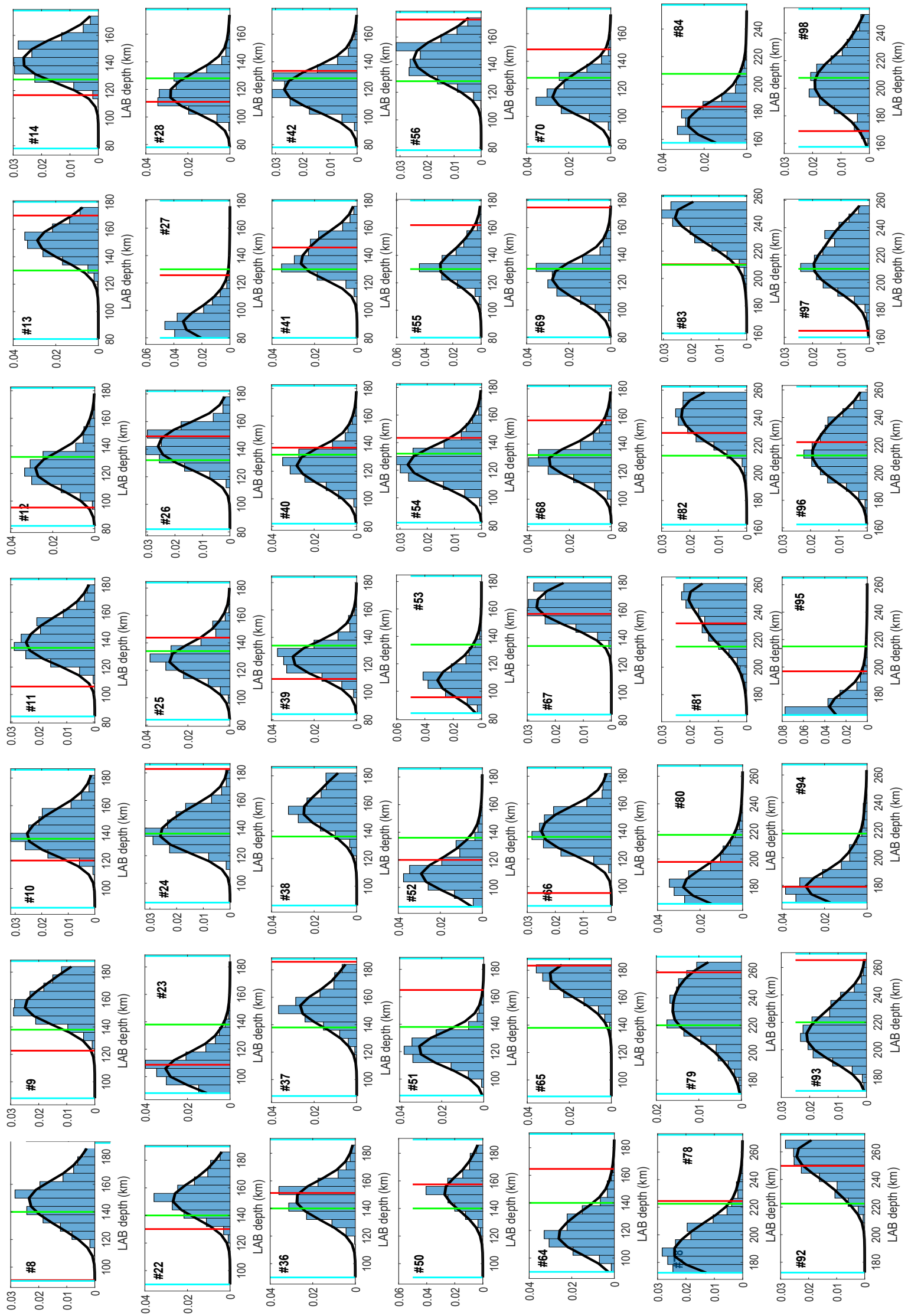

Figure 19. Marginal posterior PDFs (blue bars) and best fitting distributions (black line) of 49 of the 196 model parameters obtained after 2,500,000 RB+MCMC simulations. The real value, starting value and prior bounds of each parameter are shown in green, red, and light blue vertical lines, respectively. The position of each PDF in the figure corresponds with the location of the 49 columns at the north-west corner of the model (Fig. 1.b in main text). 

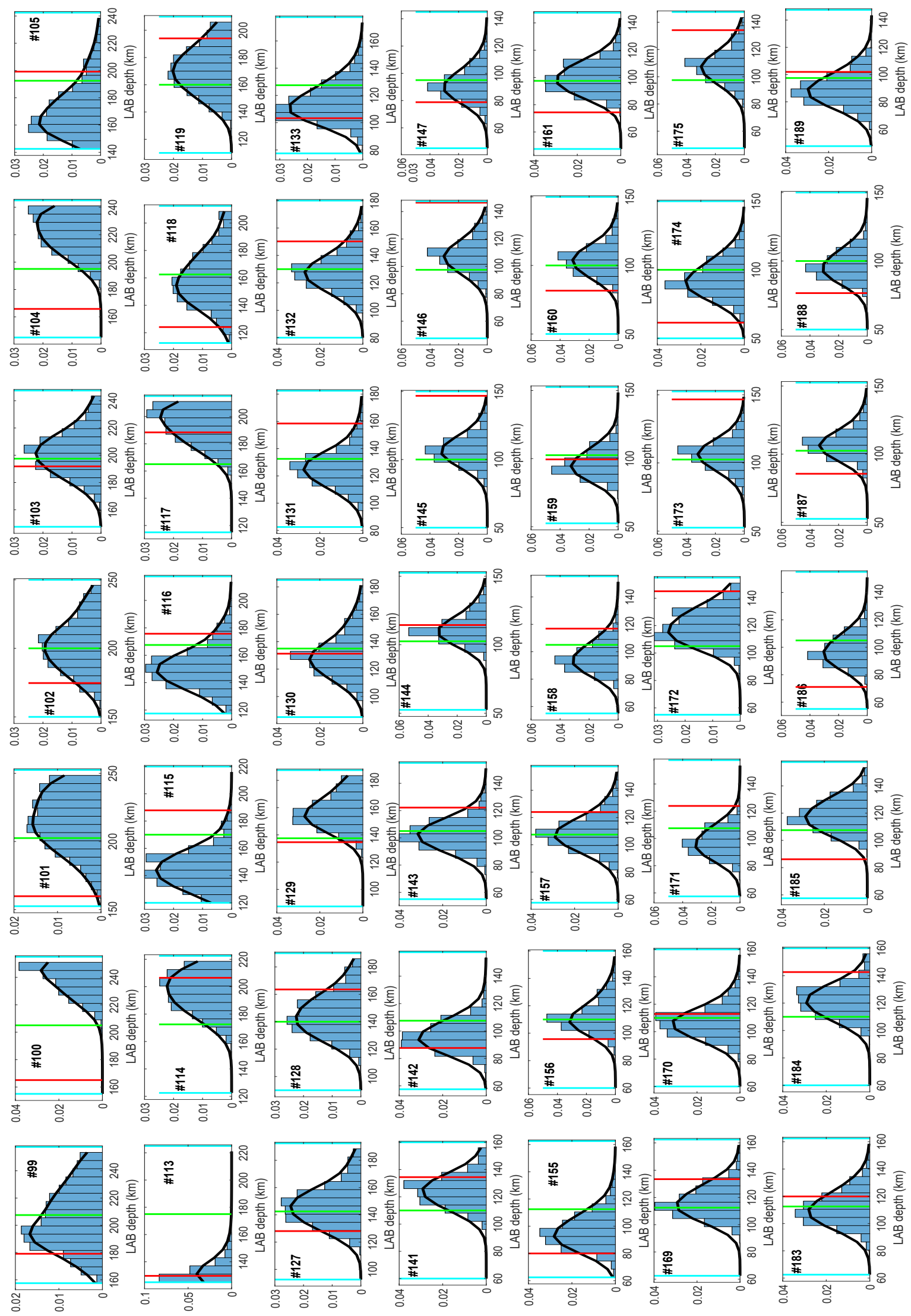

Figure 20. Marginal posterior PDFs (blue bars) and best fitting distributions (black lines) of 49 of the 196 model parameters obtained after 2,500,000 RB+MCMC simulations. The real value, starting value and prior bounds of each parameter are shown in green, red, and light blue vertical lines, respectively. The position of each PDF in the figure corresponds with the location of the 49 columns at the south-east corner of the model (Fig. 1.b in main text). 

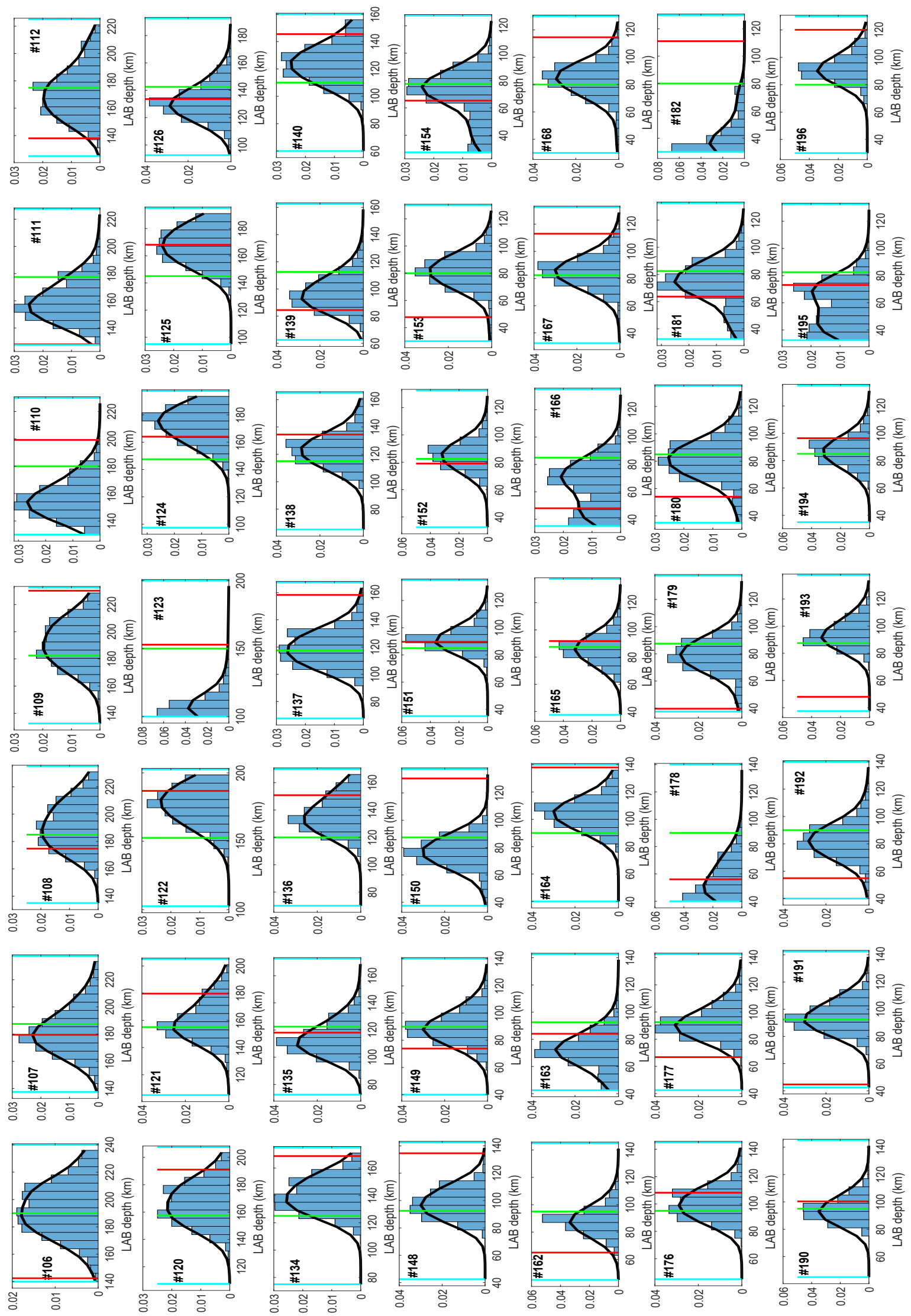

Figure 21. Marginal posterior PDFs (blue bars) and best fitting distributions (black lines) of 49 of the 196 model parameters obtained after 2,500,000 RB+MCMC simulations. The real value, starting value and prior bounds of each parameter are shown in green, red, and light blue vertical lines, respectively. The position of each PDF in the figure corresponds with the location of the 49 columns at the north-east corner of the model (Fig. 1.b in main text). 


\section{M.Constanza Manassero}

\section{NUMERICAL EXAMPLE 2: ADDITIONAL INVERSION RESULTS}

This section shows additional results of the $\mathrm{RB}+\mathrm{MCMC}$ inversion of the large-scale lithospheric model eith conductivity anomalies presented Section 6.3.

\section{Bibliography}

Egbert, G. D. \& Kelbert, A., 2012. Computational recipes for electromagnetic inverse problems, Geophysical Journal International, 189(1), 251-267.

Farquharson, C. G., Oldenburg, D. W., Haber, E., \& Shekhtman, R., 2002. An algorithm for the three-dimensional inversion of magnetotelluric data, in SEG Technical Program Expanded Abstracts 2002, pp. 649-652, Society of Exploration Geophysicists.

Mackie, R. L., Smith, J. T., \& Madden, T. R., 1994. Three-dimensional electromagnetic modeling using finite difference Equations: The magnetotelluric example, Radio Science, 29(4), 923-935.

Miensopust, M. P., Queralt, P., Jones, A. G., \& modellers, D. M., 2013. Magnetotelluric 3-D inversion-a review of two successful workshops on forward and inversion code testing and comparison, Geophysical Journal International, 193(3), 1216-1238.

Nam, M. J., Kim, H. J., Song, Y., Lee, T. J., Son, J.-S., \& Suh, J. H., 2007. 3D magnetotelluric modelling including surface topography, Geophysical Prospecting, 55(2), 277-287.

Sheen, D., 1997. Approximation of electromagnetic fields: Part I. Continuous problems, SIAM Journal on Applied Mathematics, 57(6), 1716-1736.

Siripunvaraporn, W., Egbert, G., \& Lenbury, Y., 2002. Numerical accuracy of magnetotelluric modeling: a comparison of finite difference approximations, Earth, planets and space, 54(6), 721-725. Zyserman, F. I. \& Santos, J. E., 2000. Parallel finite element algorithm with domain decomposition for three-dimensional magnetotelluric modelling, Journal of Applied Geophysics, 44(4), 337-351. 


\section{Station 334}
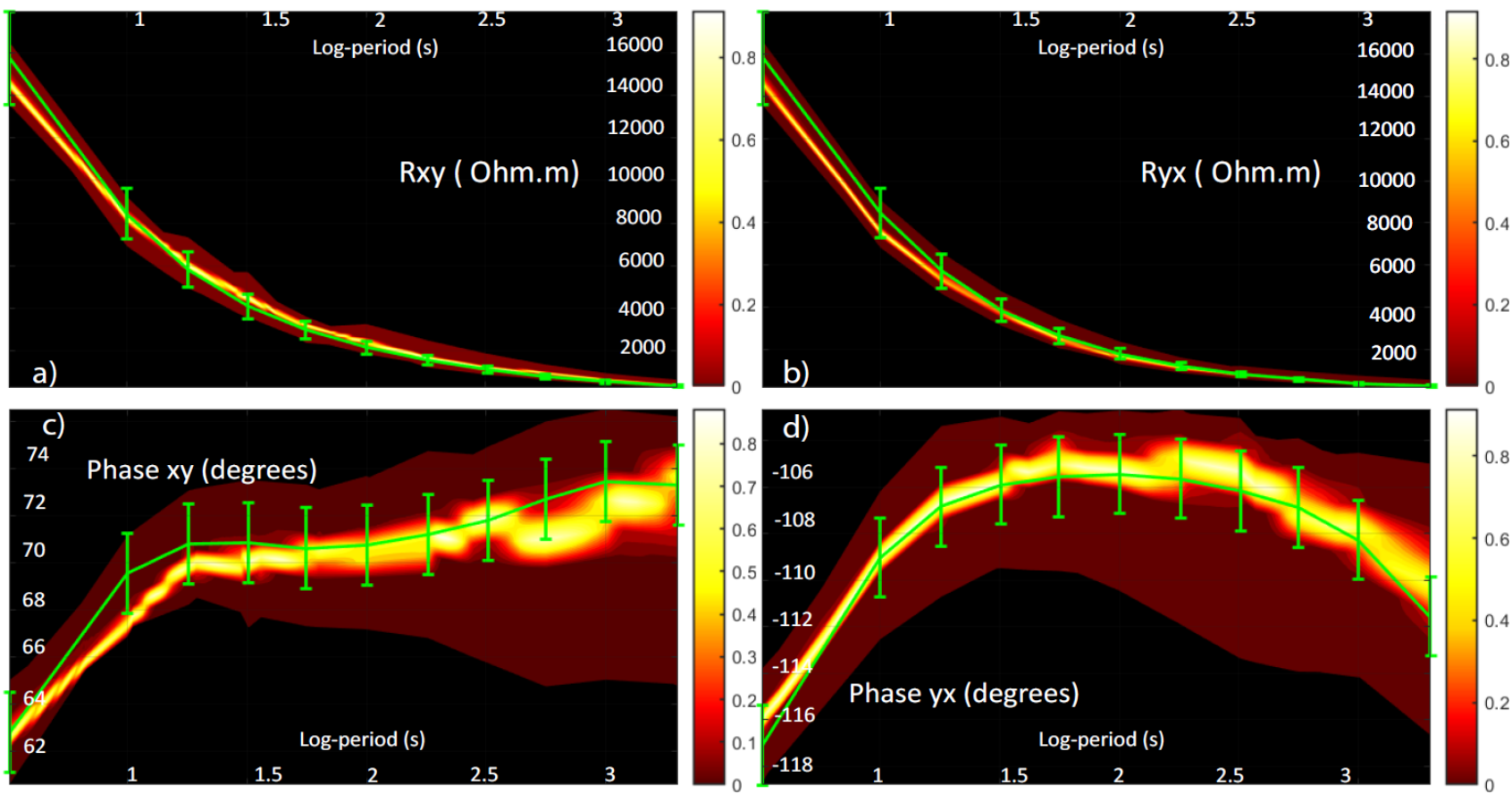

Figure 22. Posterior PDFs of data for station 334. Synthetic data and error bars are plotted in green. (a)-(b) Posterior PDFs of the off-diagonal apparent resistivity. (c)-(d) Posterior PDFs of the off-diagonal apparent phases.

\section{Station 279}
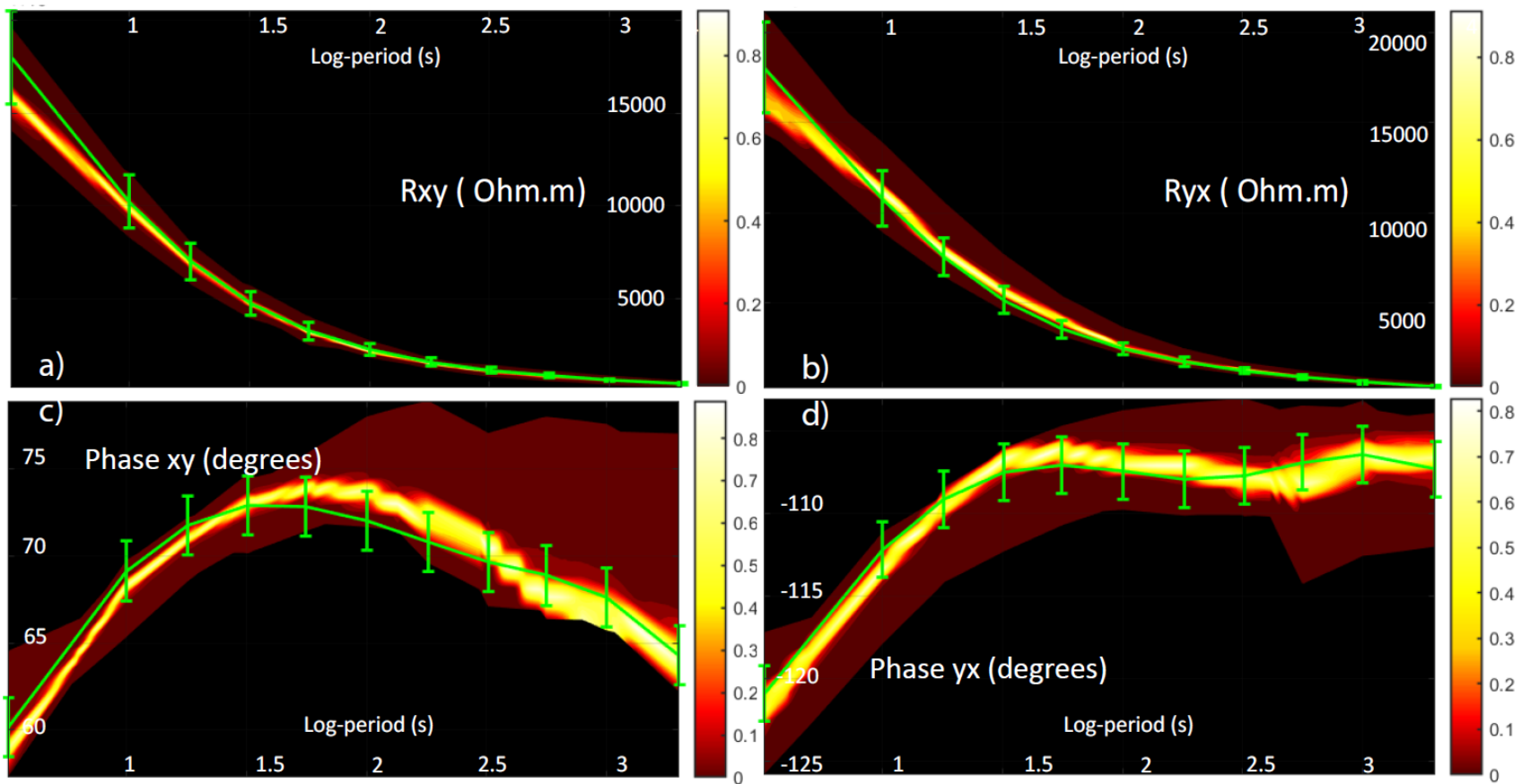

Figure 23. Posterior PDFs of data for station 279. Synthetic data and error bars are plotted in green. (a)-(b) Posterior PDFs of the off-diagonal apparent resistivity. (c)-(d) Posterior PDFs of the off-diagonal apparent phases. 


\section{M.Constanza Manassero}

\section{Station 102}
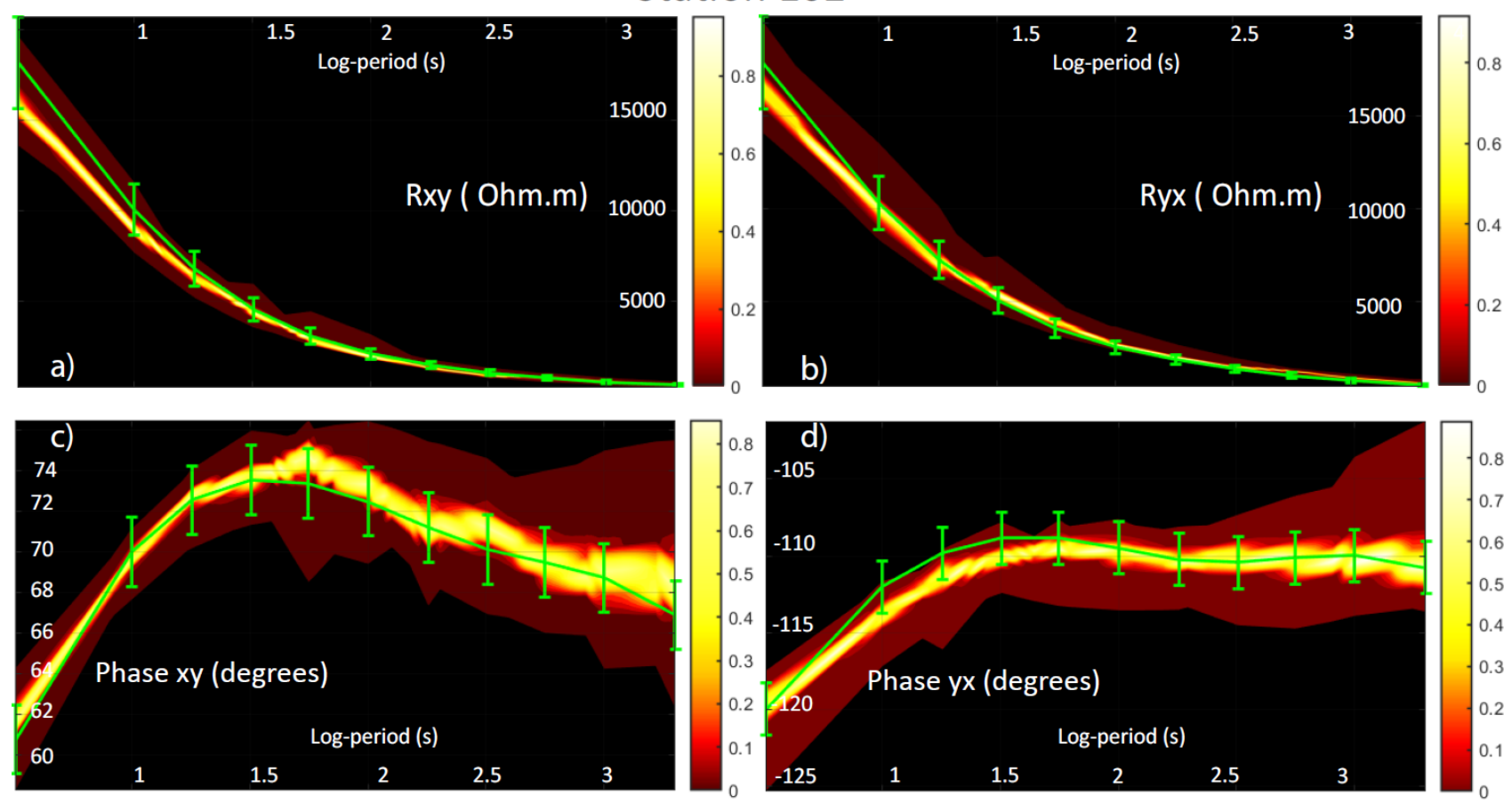

Figure 24. Posterior PDFs of data for station 102. Synthetic data and error bars are plotted in green. (a)-(b) Posterior PDFs of the off-diagonal apparent resistivity. (c)-(d) Posterior PDFs of the off-diagonal apparent phases. 

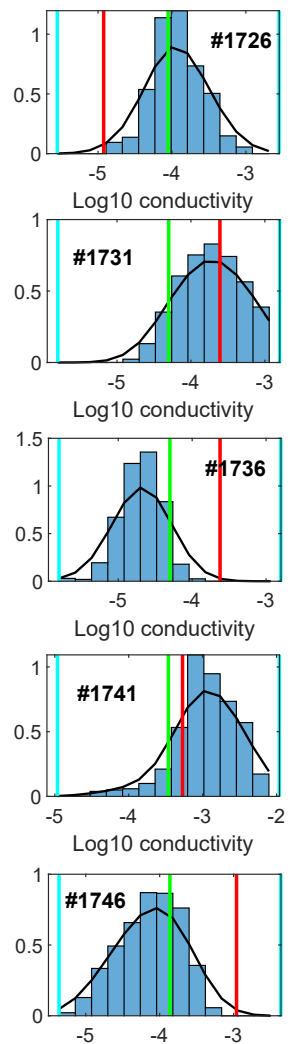

Log10 conductivity

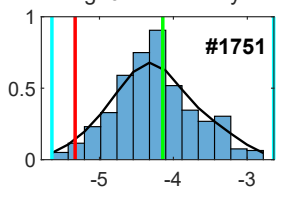

Log10 conductivity

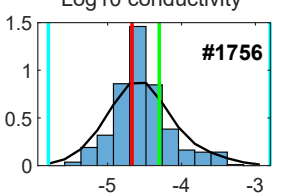

Log10 conductivity

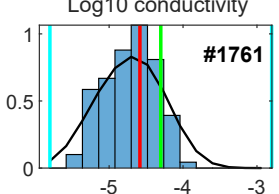

Log10 conductivity

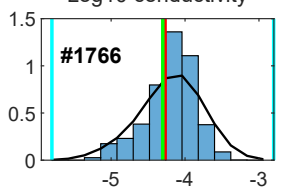

Log10 conductivity

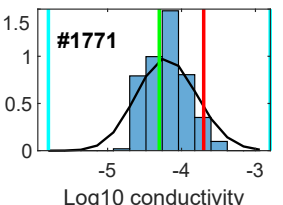

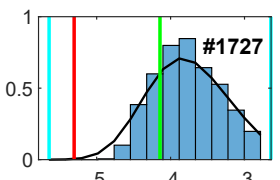
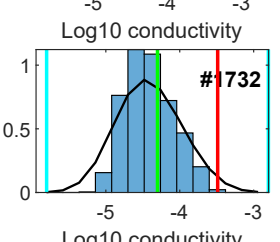

Log10 conductivity

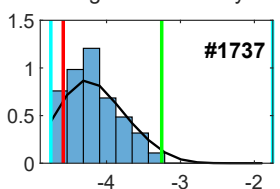

Log10 conductivity
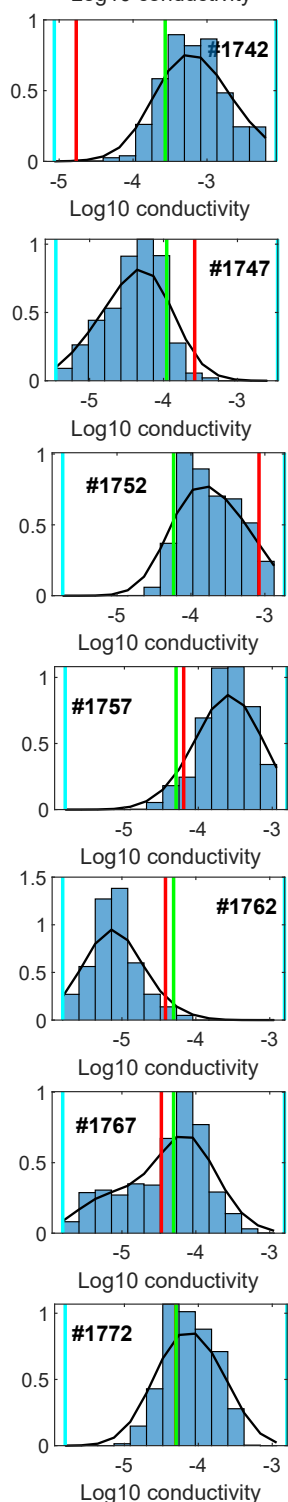
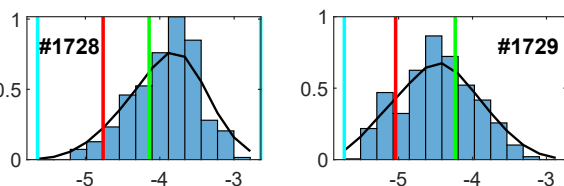

Log10 conductivity
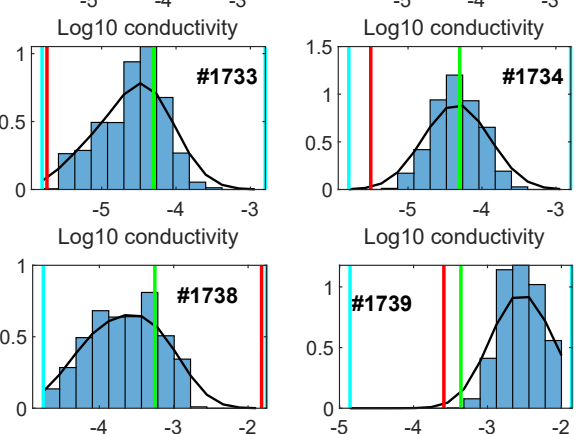

$\log _{10}^{-4}$ conductivity $^{-3}$
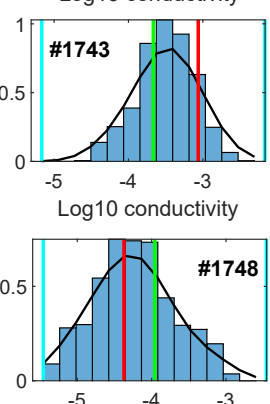

Log10 conductivity

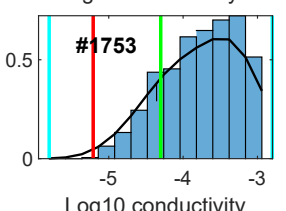

Log10 conductivity

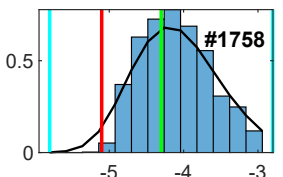

Log10 conductivity
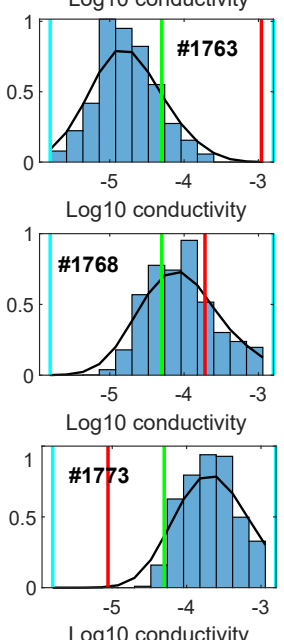
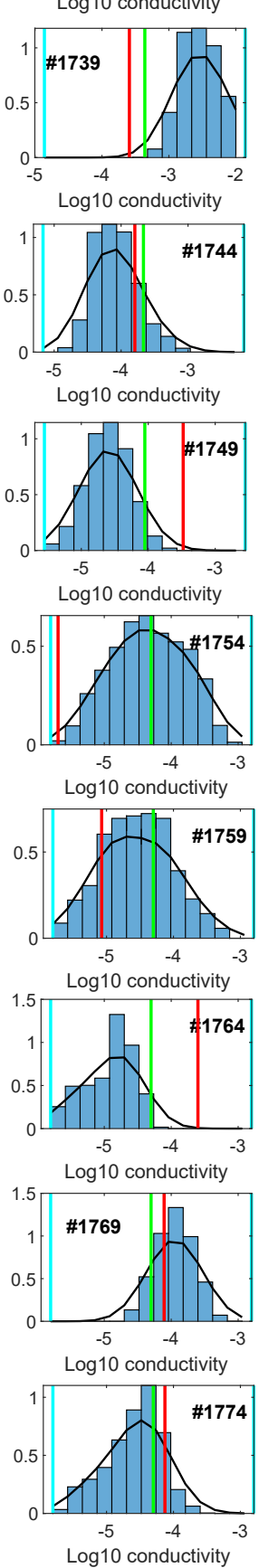

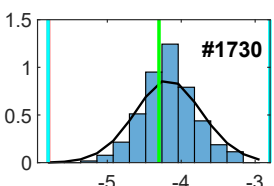

$\begin{array}{ccc}-5 & -4 & -3 \\ \operatorname{og} 10 & \text { conductivity }\end{array}$

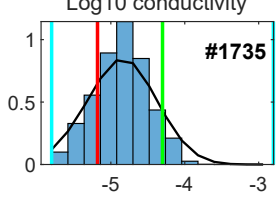

Log10 conductivity
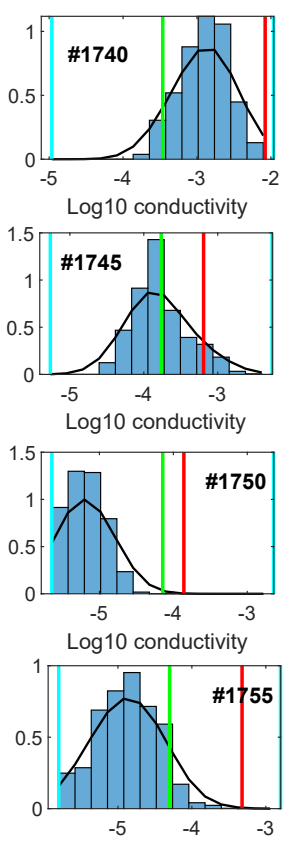

Log10 conductivity

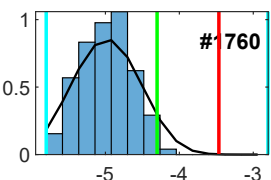

Log10 conductivity
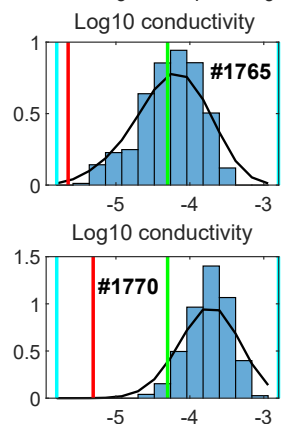

Log10 conductivity

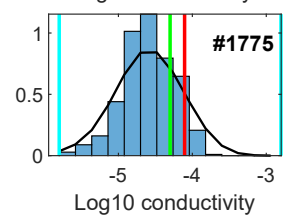

Figure 25. Marginal posterior PDFs (blue bars) and best fitting distributions (black lines) of 50 of the 1782 node-parameters obtained after 2,500,000 RB+MCMC simulations. The real value, starting value and prior bounds of each parameter are shown in green, red, and light blue vertical lines, respectively. 

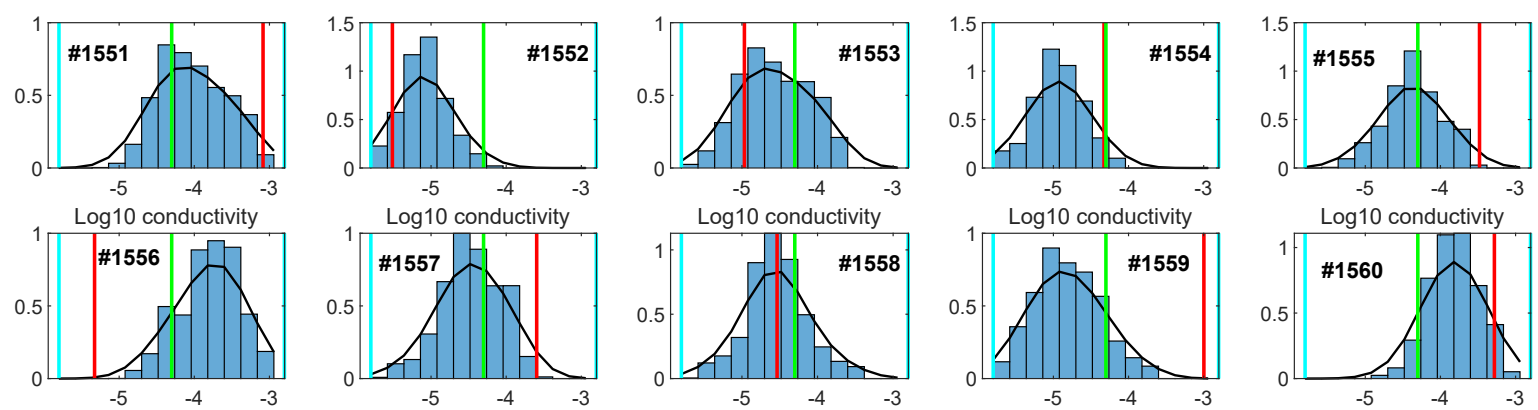

Log10 conductivity
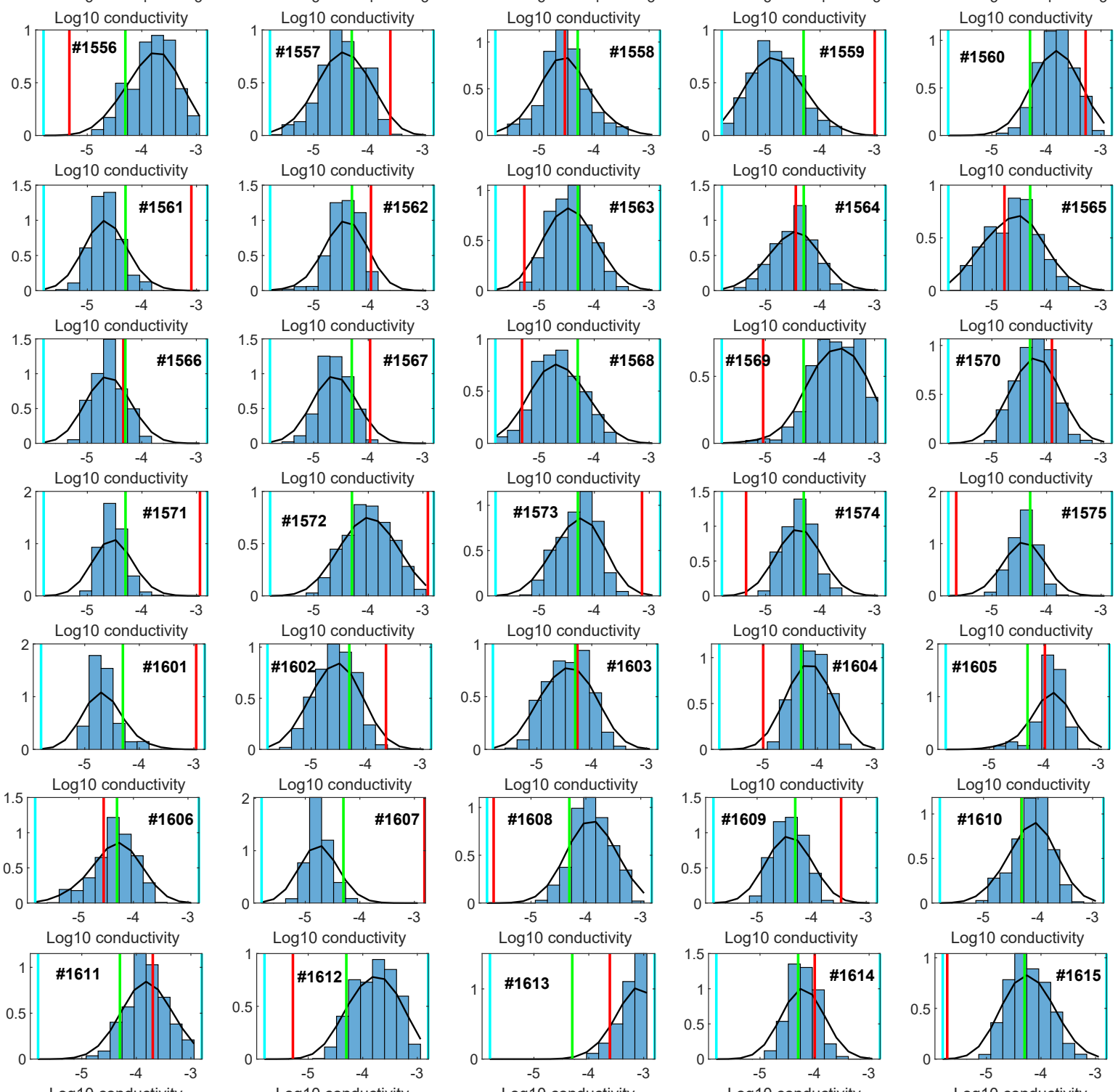

Log10 conductivity
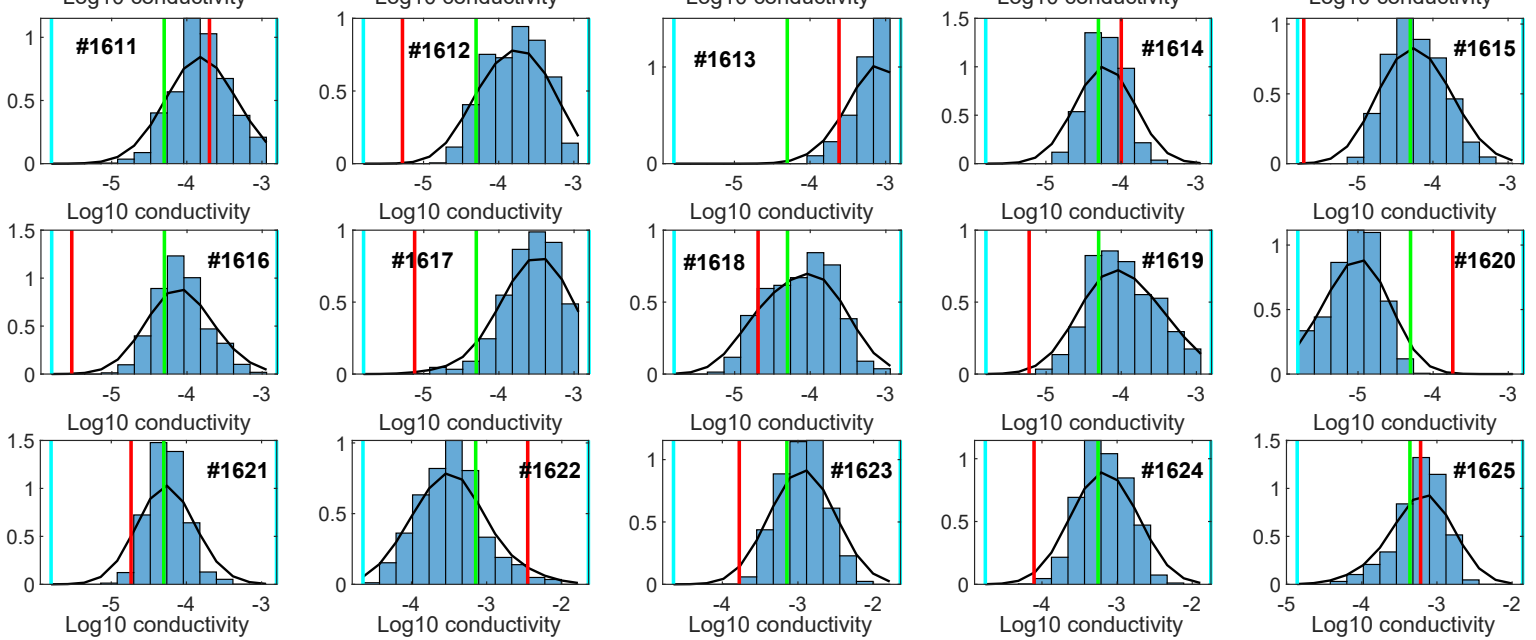

Figure 26. Marginal posterior PDFs (blue bars) and best fitting distributions (black lines) of 50 of the 1782 node-parameters obtained after 2,500,000 RB+MCMC simulations. The real value, starting value and prior bounds of each parameter are shown in green, red, and light blue vertical lines, respectively. 